\title{
ENTROPY, SHANNON ORBIT EQUIVALENCE, AND SPARSE CONNECTIVITY
}

\author{
DAVID KERR AND HANFENG LI
}

\begin{abstract}
We say that two free p.m.p. actions of countable groups are Shannon orbit equivalent if there is an orbit equivalence between them whose associated cocycle partitions have finite Shannon entropy. We show that if the acting groups are sofic and each has a w-normal amenable subgroup which is neither locally finite nor virtually cyclic then Shannon orbit equivalence implies that the actions have the same maximum sofic entropy. This extends a result of Austin beyond the finitely generated amenable setting and has the consequence that two Bernoulli actions of a group with the properties in question are Shannon orbit equivalent if and only if they are measure conjugate. Our arguments apply more generally to actions satisfying a sparse connectivity condition which we call property SC, and yield an entropy inequality under the assumption that one of the actions has this property.
\end{abstract}

\section{Contents}

1. Introduction

2. Preliminaries

2.1. Basic notation and terminology

2.2. Shannon orbit equivalence

2.3. Bounded and integrable orbit equivalence

2.4. Sofic approximations

2.5. Sofic measure entropy

3. Property SC

3.1. Definition and two permanence properties

3.2. The shrinking property

3.3. Variants of property SC

3.4. Normal subgroups and property SC

3.5. Groups without property SC

3.6. Groups with property SC

3.7. Product groups

4. Measure entropy and Shannon orbit equivalence References 


\section{INTRODUCTION}

One of the remarkable features of the notion of amenability for groups is that its fundamental characterizations in terms of nonparadoxicality on the one hand and approximate invariance on the other lead to two very different and in many ways incompatible conceptions of what its opposite should be, namely freeness and property (T) (i.e., universal spectral gap). While noncyclic free groups distill the idea of paradoxicality to its starkest form and represent the simplest and most venerable obstruction to amenability, the fact that approximate invariance affords so much technical leverage has meant that amenability is frequently contrasted instead with property $(\mathrm{T})$, even sometimes in the form of a direct counterpositioning that exploits the tension between them, as in Margulis's proof of his celebrated normal subgroup theorem? 2

In the theory of orbit equivalence, the dichotomy between approximate invariance and spectral gap plays out with particularly dramatic consequences. Here a sharp wedge is already driven between amenability and nonamenability: while the Ornstein-Weiss tiling theorem establishes that any two free ergodic p.m.p. actions of countably infinite amenable groups are orbit equivalent [29], Epstein showed, completing a line of development in [38, 15, 18, 16, 19], that every countable nonamenable group admits uncountably many orbit inequivalent free ergodic p.m.p. actions [12. At the more extreme end of spectral anti-amenability, a theorem of Popa shows that Bernoulli actions (and, more generally, weakly mixing malleable actions) of property (T) groups are in fact superrigid for cocycles taking values in a countable group, which implies, in the case that the group contains no nontrivial finite normal subgroups, that the action is orbit equivalence superrigid (i.e., an orbit equivalence with any p.m.p. action of any group implies that the groups are isomorphic and the actions measure conjugate) 31. In this sense free groups exhibit more of an affinity with amenable groups, for all nontrivial Bernoulli actions of a given free group are orbit equivalent (Bowen [7]) and all nontrivial Bernoulli actions of all noncyclic free groups are stably orbit equivalent (Bowen [8]), despite the fact that free ergodic p.m.p. actions of free groups of different ranks are never orbit equivalent (Gaboriau [15]).

Paradoxically enough, as one ventures further into the theory of Bernoulli superrigidity it is precisely around this alignment of amenability with freeness that the general picture seems to coalesce. Indeed what one discovers is that superrigidity is governed less by spectral gap per se than by certain expressions of anti-freeness or anti-treeability. To begin with, Popa's cocycle superrigidity theorem in 31] actually covers a broader class of groups, namely those containing a w-normal subgroup with relative property $(\mathrm{T})$, and was subsequently augmented in 32 by a second cocycle superrigidity theorem that gives the same conclusion for groups that contain two commuting infinite subgroups at least one of which is nonamenable. Peterson and Sinclair then demonstrated in [30] that $L^{2}$-rigidity is sufficient to imply Bernoulli cocycle superrigidity, which enlarges the menu of groups to include those which are nonamenable but have property Gamma, while Ioana and Tucker-Drob subsequently observed that nonamenable inner amenable groups

\footnotetext{
${ }^{1}$ This bifurcation is also reflected in the two logically independent ways in which the ideas of amenability and nonamenability have been adapted to operator algebras, on the one hand through the notions of finiteness and pure infiniteness and on the other through injectivity and finite-dimensional approximation. All of this traces back to the basic Dedekindian alternative for defining what it means for a set to be finite, either as the property that every injection from the set to itself is surjective, or by the existence of a bijection between the set and $\{1, \ldots, n\}$ for some positive integer $n$.

${ }^{2}$ Again this is consistent with operator algebra theory, where amenability has become synonymous with certain kinds of finite-dimensional approximation.
} 
can also be added to the list 35]. Orbit equivalence superrigidity results were also established for a variety of p.m.p. actions and groups in earlier groundbreaking papers of Furman on lattices in Lie groups [14] and of Monod and Shalom on bounded cohomology [28] as well as in work of Kida on mapping class groups that merely assumes freeness of the action [25]. One common feature of the groups that effectuate Bernoulli cocycle or orbit equivalence superrigidity in all of these cases, a feature which notably distinguishes them from noncyclic free groups, is that their first $\ell^{2}$-Betti number vanishes. Indeed Peterson and Sinclair showed in [30] that this is a necessary condition for the cocycle superrigidity of Bernoulli actions with atomless base, which has led to the speculation that it might also be sufficient within the class of nonamenable groups. The funny thing here is that amenable groups also have vanishing first $\ell^{2}$-Betti number and thus have to be explicitly ruled out. This reflects the fact a group can have vanishing first $\ell^{2}$-Betti number for two very different and incompatible reasons: either because of anti-tree-like behaviour (even when viewed through the rather coarse lens of measure equivalence) or because of tree-like behaviour of a degenerate rank-one kind (understood in the similarly generous sense of being measure equivalent to $\mathbb{Z}$, a property that characterizes amenability among countably infinite groups).

One may nevertheless wonder whether, as Robin Tucker-Drob has half-jokingly mused to us, there may be a way of reconceptualizing the idea of Bernoulli cocycle superrigidity so that it naturally extends to amenable groups. That this is not so far-fetched is suggested by the recent paper [11], which in the case of $L^{2}$-rigidity explains how amenability can be smuggled in through a perturbative maneuver. What we show in the present paper is that if we shift gears in the study of Bernoulli rigidity to the setting of what we call Shannon orbit equivalence, in which the cocycle partitions have finite Shannon entropy (as happens in a bounded or integrable orbit equivalence), then amenable groups truly do lose their exceptional status and can be reunited with some of their nonamenable brethren under the kind of common umbrella that remains a chimera in the framework of general orbit equivalence. Amenability in this case becomes largely realigned with anti-tree-like behaviour, which is now to be understood in a much stricter geometric sense. One must still exclude the virtually cyclic groups, which remain too strongly tree-like, as well as the locally finite groups (but not the locally virtually cyclic groups which fall outside of these two classes, like the rational numbers).

The basic geometric principle behind this was first identified and exploited by Austin to show that measure entropy is an invariant of integrable orbit equivalence for ergodic p.m.p. actions of finitely generated amenable groups, and more generally that there is an entropy scaling formula for stable versions of both integrable and bounded orbit equivalence [2]. In the non-virtuallycyclic case, Austin's arguments also give the same conclusions for Shannon orbit equivalence, as one can easily verify. One corollary of Austin's work, given the Ornstein-Weiss entropy classification for Bernoulli actions of countably infinite amenable groups, is that if two Bernoulli actions of a finitely generated infinite amenable group are integrably orbit equivalent (or even just Shannon orbit equivalent if the group is not virtually cyclic) then they must be measure conjugate.

Our main result, stated next and obtained as a direct consequence of Theorems 4.1 and Theorem 3.29, is a generalization of Austin's entropy invariance result to a wider class of groups and represents an initial step towards answering his Question 1.2 in [2]. Here $h_{\mu}(\cdot)$ denotes the maximum sofic measure entropy (see Section 2.5). W-normality is a weakening of normality which is recalled in Definition 3.21, 
Theorem A. Let $G$ be a countable group containing a w-normal amenable subgroup which is neither locally finite nor virtually cyclic. Let $H$ be a countable group. Let $G \curvearrowright(X, \mu)$ and $H \curvearrowright(Y, \nu)$ be free p.m.p. actions which are Shannon orbit equivalent. Then

$$
h_{\nu}(H \curvearrowright Y) \geq h_{\mu}(G \curvearrowright X) .
$$

This result is also new in the case that $G$ and $H$ are amenable and $G$ is not finitely generated. For amenable groups, the maximum sofic measure entropy is equal to the amenable measure entropy and is realized by every sofic approximation sequence [10, 23].

Note that many measure conjugacy invariants like mixing and completely positive entropy can be destroyed even under a bounded orbit equivalence, as shown by Fieldsteel and Friedman in the case of ergodic p.m.p. $\mathbb{Z}^{d}$-actions when $d \geq 2[13]$. On the other hand, by a theorem of Belinskaya two ergodic p.m.p. $\mathbb{Z}$-actions are integrably orbit equivalent if and only if they are measure conjugate up to an isomorphism of the group ("flip conjugate") [4]. We don't know however whether one can substitute "Shannon" for "integrably" in Belinskaya's theorem.

Our proof of Theorem $\mathrm{A}$ is based on the key geometric idea of [2] involving the construction of connected subgraphs which are sparse but nevertheless dense at a certain coarse scale. This accounts for the exclusion of both local finiteness and virtual cyclicity. In fact the conclusion of the theorem can fail even for bounded orbit equivalence when $G$ and $H$ are locally finite, as discovered by Vershik [36, 37] and discussed further below, and while Austin was able to conjure away the second restriction by an auxiliary argument we don't see a way to remove it here (we also note that the argument in 2] for handling virtually cyclic groups does not seem to work for Shannon orbit equivalence because of its use of an ergodic theorem). The subgraphs at play in our case will live not inside Følner sets as in [2] but rather in the phase space itself. While this permits us to cross the threshold into nonamenability, it still imposes restrictions on the group which, surely without coincidence, have steered us into the realm of vanishing first $\ell^{2}$-Betti number (see Theorem 7.2 of [26]). Indeed our strategy can be seen to fail for free groups (see Theorem 3.20). There remains however the question of whether Theorem A can be extended to other classes of groups with vanishing first $\ell^{2}$-Betti number or related anti-tree-like geometric properties, in particular the classes of groups for which Bernoulli cocycle superrigidity is known to hold. Our argument still relies heavily on amenability, but in contrast to [2] we apply it in the form of Ornstein-Weiss tiling technology.

We obtain from Theorem $\mathrm{A}$ the following consequence for Bernoulli actions. By the base entropy of a Bernoulli action $G \curvearrowright\left(X^{G}, \mu^{G}\right)$ we mean the Shannon entropy of $\mu$, i.e., the supremum of the Shannon entropies of all finite partitions of $X$. When $G$ is sofic this coincides with the sofic entropy for every sofic approximation sequence [6, 22.

Theorem B. Let $G$ and $H$ be countable sofic groups containing a w-normal amenable subgroup which is neither locally finite nor virtually cyclic. Let $G \curvearrowright\left(X^{G}, \mu^{G}\right)$ and $H \curvearrowright\left(Y^{H}, \nu^{H}\right)$ be Bernoulli actions which are Shannon orbit equivalent. Then these actions have the same base entropy.

The Ornstein-Weiss entropy classification of Bernoulli actions of countably infinite amenable groups [29] and a coinduction argument of Stepin [34] together show that if a countably infinite group contains an infinite amenable subgroup then any two of its Bernoulli actions are measure conjugate whenever they have the same base entropy (in fact this statement holds for any countably infinite group by $[9,33]$ ). Theorem $\mathrm{B}$ thus specializes to the case $G=H$ as follows. 
Theorem C. Let $G$ be a countable sofic group containing a w-normal amenable subgroup which is neither locally finite nor virtually cyclic. Then two Bernoulli actions of $G$ are Shannon orbit equivalent if and only if they are measure conjugate.

Many of the groups $G$ satisfying the hypothesis of Theorem $\mathbb{C}$ have the much stronger property that their Bernoulli actions are orbit equivalence superrigid, for example if $G$ satisfies Bernoulli cocycle superigidity and in addition has no nontrivial finite normal subgroups (see [31] or Theorem 6.16 of [24]). As mentioned above, $G$ will satisfy Bernoulli cocycle superigidity if it has property $(\mathrm{T})$, is the product of an infinite group and a nonamenable group, or is inner amenable and nonamenable, and each of these three possibilities can occur within the class of groups in Theorem [C. In particular, examples of property $(\mathrm{T})$ groups whose centre is not locally virtually cyclic can be constructed by taking products of copies of the group appearing in Example 1.7.13(iii) of [3. On the other hand, it remains an open question whether Bernoulli orbit equivalence superrigidity holds for the wreath product $\mathbb{Z} \imath F_{2}$, which is also covered by the above theorems.

To establish Theorem A, we abstract the graph-theoretic argument from [2] into a more generally applicable geometric principle that we apply in a direct way to the dynamics to yield what we call property $S C$ for both free p.m.p. actions and (by universally quantifying over such actions) groups. Given that the argument in [2] is localized to Følner sets, it might seem more natural here to follow the usual recipe and instead localize to sofic approximations, which one can do successfully in the case of topological entropy, but in testing such an approach in the measure setting we have found ourselves unable to control the empirical distribution of the sofic dynamical models ("microstates") except under special circumstances, and even then we could only derive a result for bounded orbit equivalence (one does however get some extra mileage in such circumstances, as we will show in another paper). We prove Theorem $\mathrm{A}$ by showing that its conclusion is valid assuming that the action of $G$ has property SC (Theorem 4.1) and that this hypothesis is automatic for the groups $G$ in the statement of the theorem by virtue of these $G$ having themselves property SC (Theorem 3.29).

In the case of amenable groups, we show in Proposition 3.28 that property $\mathrm{SC}$ is equivalent to the group being neither locally finite nor virtually cyclic. As mentioned above, the exclusion of local finiteness cannot be removed from Theorem A, as a theorem from Vershik's thesis demonstrates [36] (see the presentation in [37]). Indeed suppose that $G$ and $H$ are infinite locally finite groups and suppose that there are nested finite subgroups $G_{1} \subseteq G_{2} \subseteq \ldots$ of $G$ with $\bigcup_{n=1}^{\infty} G_{n}=G$ and nested finite subgroups $H_{1} \subseteq H_{2} \subseteq \ldots$ of $H$ with $\bigcup_{n=1}^{\infty} H_{n}=H$ such that $\left|G_{n}\right|=\left|H_{n}\right|$ for all $n$. Then for any two free ergodic p.m.p. actions $G \curvearrowright(X, \mu)$ and $H \curvearrowright(Y, \nu)$ there are integers $1 \leq n_{1}<n_{2}<\ldots$ and an orbit equivalence $\Psi: X \rightarrow Y$ such that $\Psi\left(G_{n_{k}} x\right)=H_{n_{k}} \Psi(x)$ for all $x \in X$ and $k \in \mathbb{N}$ (this is a special type of bounded orbit equivalence). To see this, for every $n$ consider the $\sigma$-algebra $\xi_{n}$ of $G_{n}$-invariant Borel subsets of $X$. Then $\left\{\xi_{n}\right\}$ is an ergodic homogeneous sequence in the terminology of [37. Similarly, one has the ergodic homogeneous sequence $\left\{\xi_{n}^{\prime}\right\}$ whose $n$th term is the $\sigma$-algebra of $H_{n}$-invariant Borel subsets of $Y$. Again using terminology from [37], the sequences $\left\{\xi_{n}\right\}$ and $\left\{\xi_{n}^{\prime}\right\}$ have the same type $\left\{r_{n}\right\}$, in this case given by $r_{n}=\left[G_{n}: G_{n-1}\right]=\left[H_{n}: H_{n-1}\right]$ with $G_{0}$ and $H_{0}$ denoting the trivial subgroups of $G$ and $H$, respectively. Now Corollary 1 on page 723 of [37] says that any two ergodic homogeneous sequences of the same type are lacunary isomorphic in the sense that there are integers $1 \leq n_{1}<n_{2}<\ldots$ and a measure isomorphism $\Psi: X \rightarrow Y$ which for every 
$k$ sends $\xi_{n_{k}}$ to $\xi_{n_{k}}^{\prime}$, so that $\Psi\left(G_{n_{k}} x\right)=H_{n_{k}} \Psi(x)$, as desired. We thus see in particular that if $G$ is a countably infinite locally finite group then all of the free ergodic p.m.p. actions of $G$ are boundedly orbit equivalent to each other. This includes all of the nontrivial Bernoulli actions of $G$, which exhaust the possible nonzero values of measure entropy.

We begin in Section 2 by setting up notation and reviewing basic concepts and terminology concerning orbit equivalence and sofic measure entropy (for general references on these topics see [24, 21]). In Section 3.1 we define property SC and establish two permanence properties. In Sections 3.2 and 3.3 we introduce a shrinking property and two variants of property SC which will be of subsequent technical use. In Section 3.4 we prove that property SC passes from a normal subgroup to the ambient group. In Section 3.5 we identify two classes of groups without property SC (Theorem 3.20), while Section 3.6 is dedicated to showing that the groups satisfying the hypothesis of Theorem $\mathrm{A}$ have property SC (Theorem 3.29). In Section 3.7 we derive a result on property SC that concerns product groups. Finally, we devote Section 4 to the proof of Theorem 4.1, which together with Theorem 3.29 yields Theorem A,

Acknowledgements. The first author was partially supported by NSF grant DMS-1800633. Preliminary stages of this work were carried out during his six-month stay in 2017-2018 at the ENS de Lyon, during which time he held ENS and CNRS visiting professorships and was supported by Labex MILYON/ANR-10-LABX-0070. He thanks Damien Gaboriau and Mikael de la Salle at the ENS for their generous hospitality. The second author was partially supported by NSF grants DMS-1600717 and DMS-1900746. We thank Robin Tucker-Drob for comments.

\section{Preliminaries}

2.1. Basic notation and terminology. Throughout the paper $G$ and $H$ denote countably infinite discrete groups, with identity elements $e_{G}$ and $e_{H}$ (many of our results are also valid for finite groups, usually for trivial reasons, but we make this exclusion for the convenience of forcing the measure in a free probability-measure-preserving action to be atomless, as reiterated below). We denote by $\mathcal{F}(G)$ the set of all nonempty finite subsets of $G$, and by $\overline{\mathcal{F}}(G)$ the set of symmetric finite subsets of $G$ containing $e_{G}$.

A left $F \varnothing l n e r$ sequence for the group $G$ is a sequence $\left\{F_{n}\right\}$ of nonempty finite subsets of $G$ such that $\lim _{n \rightarrow \infty}\left|g F_{n} \Delta F_{n}\right| /\left|F_{n}\right|=0$ for all $g \in G$. If $G$ admits a left Følner sequence then it is said to be amenable.

If $\mathrm{P}$ is a property then one says that a group is virtually $P$ if it has a subgroup of finite index with property $\mathrm{P}$, and locally $P$ if each of its finitely generated subgroups has property $\mathrm{P}$.

For a nonempty finite set $V$, we denote by $\mathbb{P}_{V}$ the algebra of all subsets of $V$, by $\operatorname{Sym}(V)$ the group of all permutations of $V$, and by $m$ the uniform probability measure on $V$.

By a standard probability space we mean a standard Borel space (i.e., a Polish space with its Borel $\sigma$-algebra) equipped with a probability measure. By a partition of such a space we will always mean one that is Borel (and also, if occasion demands, one that is really only a partition of a conull subset of the space). By a p.m.p. (probability-measure preserving) action of $G$ we mean an action $G \curvearrowright(X, \mu)$ of $G$ on a standard probability space by measure-preserving transformations. We express such an action using the concatenation $(g, x) \mapsto g x$ for $g \in G$ and $x \in X$ (in principle this will result in an ambiguity when two actions of the same group on the same space are at play, but in context the notation chosen for group elements will make the distinction clear). Two such actions $G \curvearrowright(X, \mu)$ and $G \curvearrowright(Y, \nu)$ are measure conjugate 
or isomorphic if there exist $G$-invariant conull sets $X_{0} \subseteq X$ and $Y_{0} \subseteq Y$ and a $G$-equivariant measure isomorphism $X_{0} \rightarrow Y_{0}$.

A Bernoulli action is a p.m.p. action of the form $G \curvearrowright\left(Y^{G}, \nu^{G}\right)$ where $(Y, \nu)$ is a standard probability space and $(g y)_{h}=y_{g^{-1} h}$ for $y \in Y^{G}$ and $g, h \in G$. It is nontrivial if $\nu$ does not have an atom with full measure.

A p.m.p. action $G \curvearrowright(X, \mu)$ is free if the set $X_{0}$ of all $x \in X$ such that $s x \neq x$ for all $s \in G \backslash\left\{e_{G}\right\}$ has measure one. For the purposes of this paper there is never any harm in replacing $X$ by a $G$-invariant conull subset and so we will always assume that $X_{0}=X$ for the purposes of argumentation, even if theorem statements themselves do not require it.

Given a p.m.p. action $G \curvearrowright(X, \mu)$ and a set $S \subseteq G$, we define an $S$-path in $X$ to be a finite tuple $\left(x_{0}, x_{1}, \ldots, x_{n}\right)$ of points in $X$ such that for every $i=1, \ldots, n$ there is a $g \in S$ for which $x_{i}=g x_{i-1}$, in which case we call $n$ the length of the path and say that the path connects $x_{0}$ and $x_{n}$. When $n=1$ we also speak of an $S$-edge.

As indicated above, our reason for making the blanket assumption that the groups $G$ and $H$ be infinite is that for any of their free p.m.p. actions on a standard probability space $(X, \mu)$ the measure $\mu$ is forced to be atomless, a fact which we will often tacitly rely on. It is required for instance in our various applications of Ornstein-Weiss tiling technology.

2.2. Shannon orbit equivalence. We say that two free p.m.p. actions $G \curvearrowright(X, \mu)$ and $H \curvearrowright$ $(Y, \nu)$ are orbit equivalent if there are a $G$-invariant conull set $X_{0} \subseteq X$, an $H$-invariant conull set $Y_{0} \subseteq Y$, and a measure isomorphism $\Psi: X_{0} \rightarrow Y_{0}$ such that $\Psi(G x)=H \Psi(x)$ for all $x \in X_{0}$. Such a $\Psi$ is called an orbit equivalence.

Associated to a $\Psi$ as in the above definition are the cocycles $\kappa: G \times X_{0} \rightarrow H$ and $\lambda: H \times Y_{0} \rightarrow$ $G$ determined by

$$
\begin{gathered}
\Psi(g x)=\kappa(g, x) \Psi(x), \\
\Psi^{-1}(t y)=\lambda(t, y) \Psi^{-1}(y)
\end{gathered}
$$

for all $g \in G, x \in X_{0}, t \in H$, and $y \in Y_{0}$. The defining property of a cocycle, referred to as the cocycle identity, is expressed in the case of $\kappa$ by

$$
\kappa(f g, x)=\kappa(f, g x) \kappa(g, x)
$$

for all $f, g \in G$ and $x \in X_{0}$. Note also that

$$
\begin{aligned}
\kappa\left(\lambda(t, y), \Psi^{-1}(y)\right) & =t, \\
\lambda(\kappa(g, x), \Psi(x)) & =g
\end{aligned}
$$

for all $t \in H, y \in Y_{0}, g \in G$, and $x \in X_{0}$.

The Shannon entropy of a countable Borel partition $\mathscr{P}$ of $X$ is defined by

$$
H_{\mu}(\mathscr{P})=\sum_{P \in \mathscr{P}}-\mu(P) \log \mu(P) .
$$

with $-x \log x$ being interpreted as 0 when $x=0$. We say that the actions are Shannon orbit equivalent if the sets $X_{0}$ and $Y_{0}$ and the measure isomorphism $\Psi$ can be chosen so that for each $g \in G$ the countable Borel partition of $X_{0}$ consisting of the sets

$$
X_{g, t}:=\left\{x \in X_{0}: \Psi(g x)=t \Psi(x)\right\}
$$


for $t \in H$ has finite Shannon entropy and, likewise, for each $t \in H$ the countable partition of $X_{0}$ consisting of the sets $X_{g, t}$ for $g \in G$ has finite Shannon entropy. In this case we refer to $\Psi$ as a Shannon orbit equivalence.

In general, we say that a map $f: X \rightarrow H$ is Shannon if the countable partition $\left\{f^{-1}(t): t \in\right.$ $H\}$ of $X$ has finite Shannon entropy. A cocycle $\kappa: G \times X \rightarrow H$ is Shannon if $\kappa(g, \cdot)$ is Shannon for every $g \in G$.

2.3. Bounded and integrable orbit equivalence. Let $G \curvearrowright(X, \mu)$ and $H \curvearrowright(Y, \nu)$ be free p.m.p. actions which are orbit equivalent, with $X_{0}, Y_{0}$, and $\Psi$ witnessing the orbit equivalence as above, and $\kappa$ and $\lambda$ denoting the associated cocycles.

We say that the cocycle $\kappa: G \times X_{0} \rightarrow H$ is bounded if $\kappa\left(g, X_{0}\right)$ is finite for every $g \in G$, and define boundedness for $\lambda$ likewise. If $X_{0}, Y_{0}$, and $\Psi$ can be chosen so that each of the cocycles $\kappa$ and $\lambda$ is bounded then we say that the actions are boundedly orbit equivalent, and refer to $\Psi$ as a bounded orbit equivalence.

Suppose now that $G$ and $H$ are finitely generated and write $\ell_{G}$ and $\ell_{H}$ for the word length functions with respect to some symmetric finite generating sets for $G$ and $H$, respectively. We say that the cocycle $\kappa: G \times X_{0} \rightarrow H$ is integrable if for every $g \in G$ one has

$$
\int_{X} \ell_{H}(\kappa(g, x)) d \mu(x)<\infty,
$$

and define integrability for $\lambda$ likewise. If $X_{0}, Y_{0}$, and $\Psi$ can be chosen so that each of the cocycles $\kappa$ and $\lambda$ is integrable then we say that the actions are integrably orbit equivalent, and refer to $\Psi$ as an integrable orbit equivalence.

Obviously every bounded orbit equivalence is integrable. Lemma 2.1 of [2] shows that every integrable orbit equivalence is Shannon.

2.4. Sofic approximations. On the set $V^{V}$ of maps from a nonempty finite set $V$ to itself we define the normalized Hamming distance by

$$
\rho_{\text {Hamm }}(T, S)=\frac{1}{|V|}|\{v \in V: T v \neq S v\}| .
$$

By a sofic approximation for $G$ we mean a (not necessarily multiplicative) map $\sigma: G \rightarrow \operatorname{Sym}(V)$ for some nonempty finite set $V$. Given a finite set $F \subseteq G$ and an $\delta>0$, we say that such a $\sigma$ is an $(F, \delta)$-approximation if

(i) $\rho_{\text {Hamm }}\left(\sigma_{s t}, \sigma_{s} \sigma_{t}\right) \leq \delta$ for all $s, t \in F$, and

(ii) $\rho_{\text {Hamm }}\left(\sigma_{s}, \sigma_{t}\right) \geq 1-\delta$ for all distinct $s, t \in F$.

By a sofic approximation sequence for $G$ we mean a sequence $\Sigma=\left\{\sigma_{k}: G \rightarrow \operatorname{Sym}\left(V_{k}\right)\right\}_{k=1}^{\infty}$ of sofic approximations for $G$ such that for every finite set $F \subseteq G$ and $\delta>0$ there is a $k_{0} \in \mathbb{N}$ such that $\sigma_{k}$ is an $(F, \delta)$-approximation for every $k \geq k_{0}$.

By saying that a sofic approximation $\sigma: G \rightarrow \operatorname{Sym}(V)$ is good enough we mean that it is an $(F, \delta)$-approximation for some finite set $F \subseteq G$ and $\delta>0$ and that this condition is sufficient for the purpose at hand.

The group $G$ is said to be sofic if it admits a sofic approximation sequence. This is the case when $G$ is amenable or residually finite, and indeed soficity can be regarded in a natural way as a simultaneous generalization of these two properties (see Section 10.2 of [24]). In particular, free groups are sofic. It remains unknown whether nonsofic groups exist. 
2.5. Sofic measure entropy. Let $G \curvearrowright(X, \mu)$ be a p.m.p. action. Let $\mathscr{C}$ be a finite partition of $X, F$ a finite subset of $G$ containing $e_{G}$, and $\delta>0$. We write $\operatorname{alg}(\mathscr{C})$ for the algebra generated by $\mathscr{C}$, consisting of all unions of members of $\mathscr{C}$, and denote by $\mathscr{C}_{F}$ the join $\bigvee_{s \in F} s \mathscr{C}$. Let $\sigma: G \rightarrow \operatorname{Sym}(V)$ be a sofic approximation for $G$. We define $\operatorname{Hom}_{\mu}(\mathscr{C}, F, \delta, \sigma)$ to be the set of homomorphisms $\varphi: \operatorname{alg}\left(\mathscr{C}_{F}\right) \rightarrow \mathbb{P}_{V}$ satisfying

(i) $\sum_{A \in \mathscr{C}} \mathrm{m}\left(\sigma_{g} \varphi(A) \Delta \varphi(g A)\right)<\delta$ for all $g \in F$, and

(ii) $\sum_{A \in \mathscr{C}_{F}}|\mathrm{~m}(\varphi(A))-\mu(A)|<\delta$.

For a finite partition $\mathscr{P} \leq \mathscr{C}$ we define $\left|\operatorname{Hom}_{\mu}(\mathscr{C}, F, \delta, \sigma)\right|_{\mathscr{P}}$ to be the cardinality of the set of restrictions of elements of $\operatorname{Hom}_{\mu}(\mathscr{C}, F, \delta, \sigma)$ to $\mathscr{P}$.

Suppose now that $G$ is sofic and let $\Sigma=\left\{\sigma_{k}: G \rightarrow \operatorname{Sym}\left(V_{k}\right)\right\}_{k=1}^{\infty}$ be a sofic approximation sequence for $G$. For a finite partition $\mathscr{P}$ of $X$ we write, notationally omitting the action for brevity,

$$
\begin{aligned}
h_{\Sigma, \mu}(\mathscr{P}, \mathscr{C}, F, \delta) & =\limsup _{k \rightarrow \infty} \frac{1}{\left|V_{k}\right|} \log \left|\operatorname{Hom}_{\mu}\left(\mathscr{C}, F, \delta, \sigma_{k}\right)\right| \mathscr{P}, \\
h_{\Sigma, \mu}(\mathscr{P}) & =\inf _{\mathscr{C} \geq \mathscr{P}} \inf _{F} \inf _{\delta>0} h_{\Sigma, \mu}(\mathscr{C}, \mathscr{P}, F, \delta)
\end{aligned}
$$

where the first infimum is over all finite partitions $\mathscr{C}$ of $X$ refining $\mathscr{P}$ and the second is over all finite sets $F \subseteq G$ containing $e_{G}$. We also write $h_{\Sigma, \mu}(G \curvearrowright X, \mathscr{P})$ for $h_{\Sigma, \mu}(\mathscr{P})$ when it is necessary to explicitly indicate the action. We define the sofic measure entropy of the action $G \curvearrowright(X, \mu)$ with respect to $\Sigma$ by

$$
h_{\Sigma, \mu}(G \curvearrowright X)=\sup _{\mathscr{P}} h_{\Sigma, \mu}(\mathscr{P}),
$$

where $\mathscr{P}$ ranges over all finite partitions of $X$.

For a p.m.p. action $G \curvearrowright(X, \mu)$ of an arbitrary $G$ we define the maximum sofic measure entropy by

$$
h_{\mu}(G \curvearrowright X)=\max _{\Sigma} h_{\Sigma, \mu}(G \curvearrowright X)
$$

where $\Sigma$ ranges over all sofic approximation sequences for $G$ (in the case that $G$ is nonsofic we interpret this maximum to be $-\infty$ ). The following proposition shows that the maximum does indeed exist.

Proposition 2.1. Suppose that $G$ is sofic. Let $G \curvearrowright(X, \mu)$ be a p.m.p. action. Then there is a sofic approximation sequence $\Pi$ for $G$ such that $h_{\Pi, \mu}(G \curvearrowright X) \geq h_{\Pi^{\prime}, \mu}(G \curvearrowright X)$ for every sofic approximation sequence $\Pi^{\prime}$ for $G$.

Proof. Put $M=\sup _{\Pi^{\prime}} h_{\Pi^{\prime}, \mu}(G \curvearrowright X)$ where $\Pi^{\prime}$ ranges over all sofic approximation sequences $\Pi^{\prime}$ for $G$. Take a sequence $\left\{\Pi_{n}=\left\{\pi_{n, k}\right\}_{k \in \mathbb{N}}\right\}_{n \in \mathbb{N}}$ of sofic approximation sequences for $G$ such that $h_{\Pi_{n}, \mu}(G \curvearrowright X) \rightarrow M$ as $n \rightarrow \infty$.

Choose an increasing sequence $F_{1} \subseteq F_{2} \subseteq \ldots$ of finite subsets of $G$ with union $G$. For each $n \in \mathbb{N}$ there exists a $K_{n} \in \mathbb{N}$ such that for every $k \geq K_{n}$ the map $\pi_{n, k}$ is an $\left(F_{n}, 1 / n\right)$ approximation for $G$. Put $\mathscr{W}=\left\{(n, k) \in \mathbb{N}^{2}: k \geq K_{n}\right\}$. Then $\mathscr{W}$ is countably infinite, and so we can take a bijection $\varphi: \mathbb{N} \rightarrow \mathscr{W}$. Put $\pi_{k}=\pi_{\varphi(k)}$ for every $k \in \mathbb{N}$ and $\Pi=\left\{\pi_{k}\right\}_{k \in \mathbb{N}}$. Then $\Pi$ is a sofic approximation sequence for $G$. For any $n \in \mathbb{N}$, any finite Borel partitions $\mathscr{C} \preceq \mathscr{U}$ of $X$, any $L \in \mathcal{F}(G)$ containing $e_{G}$, and any $\delta>0$, we have

$$
h_{\Pi, \mu}(\mathscr{C}, \mathscr{U}, L, \delta) \geq h_{\Pi_{n}, \mu}(\mathscr{C}, \mathscr{U}, L, \delta) .
$$


Thus $h_{\Pi, \mu}(G \curvearrowright X) \geq h_{\Pi_{n}, \mu}(G \curvearrowright X)$ for every $n \in \mathbb{N}$. Therefore $h_{\Pi, \mu}(G \curvearrowright X)=M$.

The measure entropy $h_{\Sigma, \mu}(G \curvearrowright X)$ is known not to depend on the choice of sofic approximation sequence $\Sigma$ in the following cases:

(i) the group $G$ is amenable, in which case we always recover the amenable measure entropy [23, 10],

(ii) the action is Bernoulli [6, 22,

(iii) the action is an algebraic action of the form $G \curvearrowright\left((\mathbb{Z} G)^{n /(\mathbb{Z} G)^{n}} A, \mu\right)$ where $A \in$ $M_{n}(\mathbb{Z} G)$ is injective as an operator on $\ell^{2}(G)^{\oplus n}$ and $\mu$ is the normalized Haar measure [17].

\section{Property SC}

A reminder that $G$ and $H$ throughout the paper are countably infinite groups, which in particular forces the measure in any of their free p.m.p. actions to be atomless.

\subsection{Definition and two permanence properties.}

Definition 3.1. Let $\mathfrak{Y}$ be a class of free p.m.p. actions of a fixed $G$. We say that $\mathfrak{Y}$ has property $S C$ ("sparsely connected") if for any function $\Upsilon: \mathcal{F}(G) \rightarrow[0, \infty)$ there exists an $S \in \overline{\mathcal{F}}(G)$ such that for any $T \in \overline{\mathcal{F}}(G)$, there are $C, n \in \mathbb{N}$, and $S_{1}, \ldots, S_{n} \in \overline{\mathcal{F}}(G)$ so that for any $G \curvearrowright(X, \mu)$ in $\mathfrak{Y}$ there are Borel sets $W, \mathcal{V}_{1}, \ldots, \mathcal{V}_{n} \subseteq X$ satisfying the following conditions:

(i) $\sum_{j=1}^{n} \Upsilon\left(S_{j}\right) \mu\left(\mathcal{V}_{j}\right) \leq 1$

(ii) $S W=X$,

(iii) if $w_{1}, w_{2} \in W$ satisfy $g w_{1}=w_{2}$ for some $g \in T$ then $w_{1}$ and $w_{2}$ are connected by a path of length at most $C$ in which each edge is an $S_{j}$-edge with both endpoints in $\mathcal{V}_{j}$ for some $1 \leq j \leq n$.

We say that a free p.m.p. action $G \curvearrowright(X, \mu)$ has property $S C$ if the singleton class containing it has property SC. We say that $G$ itself has property $S C$ if the class of all free p.m.p actions $G \curvearrowright(X, \mu)$ has property SC.

Remark 3.2. When $\mathfrak{Y}$ consists of either a single free p.m.p. action or all free p.m.p. actions of a fixed $G$, one can omit the bound $C$ since its existence is automatic, as we will verify in the paragraph following Proposition 3.5 .

We now record a couple of permanence properties. We will also later see in Section 3.4 that if $G$ has a normal subgroup with property SC then $G$ itself has property SC (Proposition 3.16) and that a prescribed finite-index subgroup of $G$ has property SC if and only if $G$ does (Proposition 3.17).

Proposition 3.3. Let $G \curvearrowright(X, \mu)$ and $H \curvearrowright(Y, \nu)$ be free p.m.p. actions which are boundedly orbit equivalent. Suppose that $G \curvearrowright(X, \mu)$ has property $S C$. Then $H \curvearrowright(Y, \nu)$ has property $S C$.

Proof. We may assume that $(X, \mu)=(Y, \nu)$ and that the identity map of $X$ provides a bounded orbit equivalence between the actions $G \curvearrowright(X, \mu)$ and $H \curvearrowright(X, \mu)$. We may also assume that both $G \curvearrowright X$ and $H \curvearrowright X$ are free. Then we have the cocycles $\kappa$ and $\lambda$ as in Section 2.2,

Let $\Upsilon_{H}$ be a function $\mathcal{F}(H) \rightarrow[0, \infty)$. Define a function $\Upsilon_{G}: \mathcal{F}(G) \rightarrow[0, \infty)$ by $\Upsilon_{G}(F)=$ $\Upsilon_{H}(\kappa(F, X))$. Since $G \curvearrowright(X, \mu)$ has property SC, there exists an $S_{G} \in \overline{\mathcal{F}}(G)$ such that for every 
$T_{G} \in \overline{\mathcal{F}}(G)$ there are $C_{G}, n_{G} \in \mathbb{N}, S_{G, 1}, \ldots, S_{G, n_{G}} \in \overline{\mathcal{F}}(G)$, and Borel subsets $W_{G}$ and $\mathcal{V}_{G, j}$ of $X$ for $1 \leq j \leq n_{G}$ satisfying the following conditions:

(i) $\sum_{j=1}^{n_{G}} \Upsilon_{G}\left(S_{G, j}\right) \mu\left(\mathcal{V}_{G, j}\right) \leq 1$

(ii) $S_{G} W_{G}=X$,

(iii) if $w_{1}, w_{2} \in W_{G}$ satisfy $g w_{1}=w_{2}$ for some $g \in T_{G}$ then $w_{1}$ and $w_{2}$ are connected by a path of length at most $C_{G}$ in which each edge is an $S_{G, j}$-edge with both endpoints in $\nu_{G, j}$ for some $1 \leq j \leq n_{G}$.

Set $S_{H}=\kappa\left(S_{G}, X\right) \in \overline{\mathcal{F}}(H)$.

Let $T_{H} \in \overline{\mathcal{F}}(H)$. Set $T_{G}=\lambda\left(T_{H}, X\right) \in \overline{\mathcal{F}}(G)$. Then we have $C_{G}, n_{G}, S_{G, j}$ for $1 \leq j \leq n_{G}$, $W$, and $\mathcal{V}_{G, j}$ for $1 \leq j \leq n_{G}$ as above. Set $C_{H}=C_{G}, n_{H}=n_{G}, S_{H, j}=\kappa\left(S_{G, j}, X\right) \in \overline{\mathcal{F}}(H)$ for $1 \leq j \leq n_{H}=n_{G}$. Also, set $W_{H}=W_{G}$ and $\mathcal{V}_{H, j}=\mathcal{V}_{G, j}$ for all $1 \leq j \leq n_{H}=n_{G}$. Then

$$
\sum_{j=1}^{n_{H}} \Upsilon_{H}\left(S_{H, j}\right) \mu\left(\mathcal{V}_{H, j}\right)=\sum_{j=1}^{n_{G}} \Upsilon_{H}\left(\kappa\left(S_{G, j}, X\right)\right) \mu\left(\mathcal{V}_{G, j}\right)=\sum_{j=1}^{n_{G}} \Upsilon_{G}\left(S_{G, j}\right) \mu\left(\mathcal{V}_{G, j}\right) \leq 1,
$$

verifying condition (i) in Definition [3.1. Note that $X=S_{G} W_{G} \subseteq S_{H} W_{G}=S_{H} W_{H}$. Thus $X=S_{H} W_{H}$, which verifies condition (ii) in Definition 3.1. Let $h \in T_{H}$ and $w_{1}, w_{2} \in W_{H}$ with $h w_{1}=w_{2}$. Then

$$
w_{2}=h w_{1}=\lambda\left(h, w_{1}\right) w_{1} \in T_{G} w_{1} .
$$

Thus $w_{1}$ and $w_{2}$ are connected by a path of length at most $C_{G}$ in which each edge is an $S_{G, j}$-edge with both endpoints in $\mathcal{V}_{G, j}$ for some $1 \leq j \leq n_{G}$. Such an edge is also an $S_{H, j}$-edge with both endpoints in $\mathcal{V}_{H, j}$. This verifies condition (iii) in Definition 3.1

Recall that a p.m.p. action $G \curvearrowright(X, \mu)$ is said to weakly contain another p.m.p. action $G \curvearrowright(Y, \nu)$ if for every finite set $F \subseteq G$, finite collection of Borel sets $B_{1}, \ldots, B_{n} \subseteq Y$, and $\delta>0$ there exist Borel sets $A_{1}, \ldots, A_{n} \subseteq X$ such that $\left|\mu\left(s_{i} A_{i} \cap A_{j}\right)-\nu\left(s B_{i} \cap B_{j}\right)\right|<\delta$ for all $s \in F$ and $1 \leq i, j \leq n$ [20, Section 10].

Proposition 3.4. Let $G \curvearrowright(Y, \nu)$ be a free p.m.p. action with property SC. Then the class $\mathfrak{Y}$ of all free p.m.p. actions $G \curvearrowright(X, \mu)$ which weakly contain $G \curvearrowright(Y, \nu)$ has property $S C$.

Proof. Let $\Upsilon$ be a function $\mathcal{F}(G) \rightarrow[0, \infty)$. Since $G \curvearrowright(Y, \nu)$ has property SC, there is an $S \in \overline{\mathcal{F}}(G)$ such that for any $T \in \overline{\mathcal{F}}(G)$ there are $C, n \in \mathbb{N}$ and $S_{1}, \ldots, S_{n} \in \overline{\mathcal{F}}(G)$ and Borel sets $W, \mathcal{V}_{j} \subseteq Y$ for $1 \leq j \leq n$ satisfying the following conditions:

(i) $\sum_{j=1}^{n} 2 \Upsilon\left(S_{j}\right) \nu\left(\mathcal{V}_{j}\right) \leq 1$,

(ii) $S W=Y$,

(iii) if $w_{1}, w_{2} \in W$ satisfy $t w_{1}=w_{2}$ for some $t \in T$ then $w_{1}$ and $w_{2}$ are connected by a path of length at most $C$ in which every edge is an $S_{j}$-edge with both endpoints in $\mathcal{V}_{j}$ for some $1 \leq j \leq n$.

Let $T \in \overline{\mathcal{F}}(G)$. Then we have $C, n, S_{1}, \ldots, S_{n}, W, \mathcal{V}_{1}, \ldots, \mathcal{V}_{n}$ as above. Write $[n]=\{1, \ldots, n\}$ and $[C]=\{1, \ldots, C\}$ for brevity. Denote by $\Xi$ the set of $(f, h)$ such that $f$ is a function $[C] \rightarrow[n]$ and $h$ is a function $[C] \rightarrow G$ satisfying $h(j) \in S_{f(j)}$ for all $j \in[C]$. For each $t \in T$, denote by $\Xi_{t}$ the set of $(f, h) \in \Xi$ satisfying $h(C) h(C-1) \ldots h(1)=t$. For each $(f, h) \in \Xi$, denote by $Y_{f, h}$ the set of $y \in Y$ satisfying $h(j-1) \ldots h(1) y, h(j) h(j-1) \ldots h(1) y \in \mathcal{V}_{f(j)}$ for all $j \in[C]$. Then the above condition (iii) means that for every $t \in T$ the set $W \cap t^{-1} W$ is contained in $\bigcup_{(f, h) \in \Xi_{t}} Y_{f, h}$. 

$1 / 2$.

Put $S_{n+1}=T \in \overline{\mathcal{F}}(G)$. Take $0<\delta<1$ such that $\delta\left(\Upsilon(T)|T|(2|T|+2 C|\Xi|)+\sum_{j=1}^{n} \Upsilon\left(S_{j}\right)\right) \leq$

Let $G \curvearrowright(X, \mu)$ be an action in $\mathfrak{Y}$. Because it weakly contains $G \curvearrowright(Y, \nu)$, there are Borel sets $W^{\prime} \subseteq X, \mathcal{V}_{j}^{\prime} \subseteq X$ for $j \in[n]$, and $X_{f, h} \subseteq X$ for $(f, h) \in \Xi$ satisfying the following conditions:

(i) $\left|\mu\left(S W^{\prime}\right)-\nu(S W)\right|<\delta$,

(ii) $\left|\mu\left(\mathcal{V}_{j}^{\prime}\right)-\nu\left(\mathcal{V}_{j}\right)\right|<\delta$ for all $j \in[n]$,

(iii) $\mu\left(h(j-1) \ldots h(1) X_{f, h} \backslash \mathcal{V}_{j}^{\prime}\right), \mu\left(h(j) h(j-1) \ldots h(1) X_{f, h} \backslash \mathcal{V}_{j}^{\prime}\right)<\delta$ for all $(f, h) \in \Xi$ and $j \in[C]$,

(iv) $\mu\left(\left(W^{\prime} \cap t^{-1} W^{\prime}\right) \backslash \bigcup_{(f, h) \in \Xi_{t}} X_{f, h}\right)<\delta$ for each $t \in T$.

Put $W^{\prime \prime}=W^{\prime} \cup\left(X \backslash S W^{\prime}\right)$. Then $S W^{\prime \prime}=X$, verifying condition (ii) in Definition 3.1.

For each $(f, g) \in \Xi$, denote by $X_{f, h}^{\prime}$ the set of $x \in X_{f, h}$ satisfying $h(j-1) \ldots h(1) x, h(j) h(j-$ 1) ...h(1) $x \in \mathcal{V}_{f(j)}^{\prime}$ for all $j \in[C]$. Then $\mu\left(X_{f, h} \backslash X_{f, h}^{\prime}\right)<2 C \delta$.

For each $t \in T$, put $W_{t}^{\dagger}=\left(W^{\prime} \cap t^{-1} W^{\prime}\right) \backslash \bigcup_{(f, h) \in \Xi_{t}} X_{f, h}^{\prime}$. Then

$$
\begin{aligned}
\mu\left(\bigcup_{t \in T} W_{t}^{\dagger}\right) & \leq \sum_{t \in T} \mu\left(W_{t}^{\dagger}\right) \\
& \leq \sum_{t \in T} \mu\left(\left(W^{\prime} \cap t^{-1} W^{\prime}\right) \backslash \bigcup_{(f, h) \in \Xi_{t}} X_{f, h}\right)+\sum_{t \in T} \sum_{(f, h) \in \Xi_{t}} \mu\left(X_{f, h} \backslash X_{f, h}^{\prime}\right) \\
& <|T| \delta+2 C|\Xi| \delta .
\end{aligned}
$$

Put $\mathcal{V}_{n+1}^{\prime}=T\left(T\left(X \backslash S W^{\prime}\right) \cup \bigcup_{t \in T} W_{t}^{\dagger}\right) \subseteq X$. Then

$$
\begin{aligned}
\sum_{j=1}^{n+1} \Upsilon\left(S_{j}\right) \mu\left(\mathcal{V}_{j}^{\prime}\right) & \leq \Upsilon\left(S_{n+1}\right) \mu\left(\mathcal{V}_{n+1}^{\prime}\right)+\sum_{j=1}^{n} \Upsilon\left(S_{j}\right)\left(\nu\left(\mathcal{V}_{j}\right)+\delta\right) \\
& \leq \Upsilon(T)|T|(|T| \delta+|T| \delta+2 C|\Xi| \delta)+\frac{1}{2}+\delta \sum_{j=1}^{n} \Upsilon\left(S_{j}\right) \leq 1
\end{aligned}
$$

verifying condition (i) in Definition 3.1 .

Let $t \in T$ and $w_{1}, w_{2} \in W^{\prime \prime}$ with $t w_{1}=w_{2}$. If $w_{1} \in X_{f, h}^{\prime}$ for some $(f, h) \in \Xi_{t}$, then $w_{1}$ and $w_{2}$ are connected by the path $w_{1}, h(1) w_{1}, \ldots, h(C) \ldots h(1) w_{1}=t w_{1}=w_{2}$ of length $C$ whose $j$ th edge is an $S_{f(j)}$-edge with both endpoints in $\mathcal{V}_{f(j)}^{\prime}$ for all $1 \leq j \leq C$. Thus we may assume that $w_{1} \in\left(W^{\prime \prime} \cap t^{-1} W^{\prime \prime}\right) \backslash \bigcup_{(f, h) \in \Xi_{t}} X_{f, h}^{\prime} \subseteq\left(X \backslash S W^{\prime}\right) \cup t^{-1}\left(X \backslash S W^{\prime}\right) \cup W_{t}^{\dagger}$. It follows that $\left(w_{1}, w_{2}\right)$ is an $S_{n+1}$-edge with both endpoints in $\mathcal{V}_{n+1}^{\prime}$. This verifies condition (iii) in Definition [3.1,

Using the above proposition we derive the following.

Proposition 3.5. The following conditions are equivalent:

(i) $G$ has property $S C$,

(ii) every free p.m.p. action $G \curvearrowright(X, \mu)$ has property $S C$,

(iii) there exists a nontrivial Bernoulli action of $G$ with property $S C$.

Proof. (i) $\Rightarrow($ ii $) \Rightarrow($ iii). Trivial. 
(iii) $\Rightarrow$ (i). Combine Proposition 3.4 with the theorem of Abért and Weiss that every free p.m.p. action of $G$ weakly contains every nontrivial Bernoulli action of $G$ [1].

We can now verify that the existence of the bound $C$ in Definition 3.1 is automatic when $\mathfrak{Y}$ consists of either a single free p.m.p. action or all free p.m.p. actions of a fixed $G$. It suffices to check the case of a single free p.m.p. action $G \curvearrowright(X, \mu)$ in view of the implication (iii) $\Rightarrow$ (i) in Proposition 3.5. Suppose then that the action $G \curvearrowright(X, \mu)$ satisfies the weaker formulation that omits the $C$ and suppose that we are given $S, T, n, S_{j}$ for $1 \leq j \leq n$, and $W$ and $\mathcal{V}_{j}$ for $1 \leq j \leq n$ satisfying the weaker condition with respect to the function $2 \Upsilon$. For each $g \in T$ and $C \in \mathbb{N}$ we define $W_{g, C}$ to be the set of all $w_{1} \in W \cap g^{-1} W$ such that $w_{1}$ and $g w_{1}$ can be connected by a path of length at most $C$ in which each edge is an $S_{j}$-edge with both endpoints in $\mathcal{V}_{j}$ for some $1 \leq j \leq n$. Then $W \cap g^{-1} W$ is the increasing union of the sets $W_{g, C}$ for $C \in \mathbb{N}$. Thus given $\tau>0$ we can find some $C \in \mathbb{N}$ such that $\mu\left(\left(W \cap g^{-1} W\right) \backslash W_{g, C}\right)<\tau$ for all $g \in T$. Set $W^{\dagger}=W \backslash \bigcup_{g \in T}\left(\left(W \cap g^{-1} W\right) \backslash W_{g, C}\right), S_{n+1}=T$, and $\mathcal{V}_{n+1}=T\left(W \backslash W^{\dagger}\right)$. Provided that $\tau$ is small enough so that $\Upsilon(T)|T|^{2} \tau \leq 1 / 2$, we then have

$$
\sum_{j=1}^{n+1} \Upsilon\left(S_{j}\right) \mu\left(\mathcal{V}_{j}\right) \leq \frac{1}{2}+\Upsilon\left(S_{n+1}\right) \mu\left(\mathcal{V}_{n+1}\right) \leq \frac{1}{2}+\Upsilon(T)|T|^{2} \tau \leq 1
$$

Let $g \in T$ and $w_{1}, w_{2} \in W$ with $g w_{1}=w_{2}$. If $w_{1} \in W^{\dagger}$ then $w_{1} \in W_{g, C}$, in which case $w_{1}$ and $w_{2}$ are connected by a path of length at most $C$ in which each edge is an $S_{j}$-edge with both endpoints in $\mathcal{V}_{j}$ for some $1 \leq j \leq n$. If on the other hand $w_{1} \notin W^{\dagger}$, then $\left(w_{1}, w_{2}\right)$ is an $S_{n+1}$-edge with both endpoints in $\mathcal{V}_{n+1}$.

3.2. The shrinking property. The shrinking property introduced here will be of technical value in the proofs of Proposition 3.16 and Lemma 3.26 (both via Proposition 3.15) as well as Lemma 3.30 and Proposition 3.32, For the purpose of these applications we provide a characterization of when it holds in Proposition 3.11. Notice that, within the chain of quantification, the position of the sets $S_{1}$ (acting as the generator of a graph in which the path in (iii) lives) and $S$ (expressing the "scale" at which the set $W$ is dense in $X$ ) is a reversal of what occurs in the definition of property SC. The proof of Proposition 3.16 gives an illustration of how this can be leveraged in direct conjunction with property SC.

Definition 3.6. We say that $G$ has the shrinking property if there is an $S_{1} \in \overline{\mathcal{F}}(G)$ such that for every $\varepsilon>0$ there is an $S \in \overline{\mathcal{F}}(G)$ so that for every $\delta>0$ there is a $C \in \mathbb{N}$ such that given any free p.m.p. action $G \curvearrowright(X, \mu)$ we can find Borel sets $Z \subseteq \mathcal{V} \subseteq X$ satisfying the following conditions:

(i) $S \mathcal{V}=X$,

(ii) $\mu(\mathcal{V}) \leq \varepsilon$ and $\mu(Z) \leq \delta$,

(iii) every point of $\mathcal{V}$ is connected to some point of $Z$ by an $S_{1}$-path of length at most $C$ whose points all belong to $\mathcal{V}$.

Lemma 3.7. Suppose that $s \in G$ generates an infinite subgroup $G_{1}$ such that $G / G_{1}$ is finite. Let $B$ be a subset of $G$ containing $e_{G}$ such that $G=\bigsqcup_{b \in B} G_{1} b$. For each $n \in \mathbb{N}$ set $F_{n}=\left\{s^{j}\right.$ : $-n \leq j \leq n\} B$. Then $\left\{F_{n}\right\}_{n \in \mathbb{N}}$ is a left Følner sequence for $G$. 
Proof. Since $G_{1}$ has finite index in $G$ and $G$ is finitely generated, $G_{1}$ has a subgroup $G_{0}$ such that $G_{0}$ is normal and has finite index in $G$. Then $G_{0}$ is generated by $s^{N}$ for some $N \in \mathbb{N}$.

For each $n \in \mathbb{N}$, put $A_{n}=\left\{s^{N j}:-n \leq j \leq n\right\} \in \mathcal{F}\left(G_{0}\right)$. For each $g \in G$, the map $x \mapsto g x g^{-1}$ is an automorphism of $G_{0} \cong \mathbb{Z}$ and hence either $g x g^{-1}=x$ for all $x \in G_{0}$ or $g x g^{-1}=x^{-1}$ for all $x \in G_{0}$, which implies that $g A_{n} g^{-1}=A_{n}$ for every $n \in \mathbb{N}$.

Let $K \in \mathcal{F}(G)$. Then we can find some $M \in \mathbb{N}$ such that $K F_{N} B \subseteq F_{M N}$. For any $n \in \mathbb{N}$ satisfying $n \geq N$, writing $n$ as $k N+m$ for some $0 \leq m \leq N-1$ and $k \in \mathbb{N}$ we have

$$
K F_{n} \subseteq K F_{N} A_{k} B=A_{k} K F_{N} B \subseteq A_{k} F_{M N}=F_{(M+k) N}
$$

and hence $\left|K F_{n}\right| \leq\left|F_{(M+k) N}\right|=\frac{2(M+k) N+1}{2 k N+1}\left|F_{k N}\right| \leq \frac{2(M+k) N+1}{2 k N+1}\left|F_{n}\right|$. As $n \rightarrow \infty$ we have $k \rightarrow \infty$, whence $\frac{2(M+k) N+1}{2 k N+1} \rightarrow 1$. Therefore $\left\{F_{n}\right\}_{n \in \mathbb{N}}$ is a left Følner sequence for $G$.

Lemma 3.8. Suppose that $G$ is locally virtually cyclic but neither locally finite nor virtually cyclic. Then $G$ has both property $S C$ and the shrinking property.

Proof. Since $G$ is not locally finite, it has a finitely generated infinite subgroup $G_{2}$. As $G$ is locally virtually cyclic, $G_{2}$ is virtually cyclic and hence contains a subgroup $G_{1}$ such that $G_{1}$ is isomorphic to $\mathbb{Z}$ and has finite index in $G_{2}$.

Take an increasing sequence $\left\{U_{n}\right\}_{n \in \mathbb{N}}$ of finite subsets of $G$ with union $G$. Let $n \geq 3$. Denote by $G_{n}$ the subgroup of $G$ generated by $G_{2}$ and $U_{n}$. Then $G_{n}$ is finitely generated, and hence has a finite-index cyclic subgroup $G_{n}^{\prime}$. Since $G_{1}$ is infinite and $G_{n}^{\prime}$ has finite index in $G_{n}$, the intersection $G_{1} \cap G_{n}^{\prime}$ must be nontrivial and hence have finite index in $G_{n}^{\prime}$. Then $G_{1} \cap G_{n}^{\prime}$ has finite index in $G_{n}$, whence $G_{1}$ has finite index in $G_{n}$.

Now we have that $\left\{G_{n}\right\}_{n \in \mathbb{N}}$ is an increasing sequence of proper subgroups of $G$ with union $G, G_{1}$ is isomorphic to $\mathbb{Z}$, and $G_{1}$ has finite index in $G_{n}$ for every $n \in \mathbb{N}$. Then $\left[G_{n}: G_{1}\right] \rightarrow \infty$ as $n \rightarrow \infty$. Take an $s \in G_{1}$ generating $G_{1}$.

Put $S_{1}=\left\{s, e_{G}, s^{-1}\right\} \in \overline{\mathcal{F}}(G)$. Let $\Upsilon$ be a function $\mathcal{F}(G) \rightarrow[0, \infty)$. Take $m \in \mathbb{N}$ such that $\Upsilon\left(S_{1}\right) \leq\left[G_{m}: G_{1}\right] / 6$. Since $G_{1}$ has finite index in $G_{m}, G_{1}$ has a subgroup $G_{0}$ such that $G_{0}$ is a finite-index normal subgroup of $G_{m}$. Then $G_{0}$ is generated by $s^{N}$ for some $N \in \mathbb{N}$.

For each $n \in \mathbb{N}$, put $A_{n}=\left\{s^{N j}:-n \leq j \leq n\right\} \in \mathcal{F}\left(G_{0}\right)$. For each $g \in G_{m}$, the map $x \mapsto g x g^{-1}$ is an automorphism of $G_{0} \cong \mathbb{Z}$ and hence either $g x g^{-1}=x$ for all $x \in G_{0}$ or $g x g^{-1}=x^{-1}$ for all $x \in G_{0}$, which implies that $g A_{n} g^{-1}=A_{n}$ for every $n \in \mathbb{N}$.

Take a $B \in \mathcal{F}\left(G_{m}\right)$ containing $e_{G}$ such that $G_{m}=\bigsqcup_{b \in B} G_{1} b$. For each $n \in \mathbb{N}$ put $K_{n}=\left\{s^{j}\right.$ : $-n \leq j \leq n\} \in \mathcal{F}\left(G_{1}\right)$. Also, put $S=\left(K_{N} B\right) \cup\left(K_{N} B\right)^{-1} \in \overline{\mathcal{F}}\left(G_{m}\right)$.

Let $T \in \overline{\mathcal{F}}(G)$. Take an $M \in \mathbb{N}$ bigger than $m$ such that $T \subseteq G_{M}$. Take a $D \in \mathcal{F}\left(G_{M}\right)$ containing $e_{G}$ such that $G_{M}=\bigsqcup_{d \in D} G_{m} d$. Put $S_{2}=D^{-1} D \in \overline{\mathcal{F}}\left(G_{M}\right)$ and $S_{3}=T \in \overline{\mathcal{F}}\left(G_{M}\right)$. Take $\tau>0$ with $\tau|T| \Upsilon(T) \leq 1 / 3$, and take $0<\eta<\min \{1 / 2, \tau /(4|S|+1)\}$.

For $F \in \mathcal{F}\left(G_{M}\right)$ write $\partial_{T} F$ for the set $\{g \in F: T g \nsubseteq F\}$. For each $n \in \mathbb{N}$ put $F_{n}=K_{n} B D \in$ $\mathcal{F}\left(G_{M}\right)$. From Lemma 3.7 we know that $\left\{F_{n N}\right\}_{n \in \mathbb{N}}$ is an increasing left Følner sequence for $G_{M}$. Then we can find some $n_{0} \in \mathbb{N}$ such that $\Upsilon\left(S_{2}\right) /\left(2 n_{0} N+1\right)<1 / 6$ and for any $n \geq n_{0}$ one has $\left|\partial_{T} F_{n N}\right| \leq \eta\left|F_{n N}\right|$. By the Ornstein-Weiss quasitower theorem [24, Theorem 4.44] there are some $l \in \mathbb{N}$ and $n_{0}<n_{1}<n_{2}<\cdots<n_{l}$ in $\mathbb{N}$ such that for any free p.m.p. action $G \curvearrowright(X, \mu)$ there are Borel sets $Z_{1}, \ldots, Z_{l} \subseteq X$ satisfying the following conditions: 
(i) for each $1 \leq j \leq l$ there is a set $F_{n_{j} N, x} \subseteq F_{n_{j} N}$ depending measurably on $x \in Z_{j}$ and satisfying $\left|F_{n_{j} N, x}\right| \geq(1-\eta /|T|)\left|F_{n_{j} N}\right|$ for each $x \in Z_{j}$ so that the sets $F_{n_{j} N, x} x$ for $x \in Z_{j}$ are pairwise disjoint,

(ii) $F_{n_{i} N} Z_{i} \cap F_{n_{j} N} Z_{j}=\emptyset$ for $i \neq j$,

(iii) $\mu\left(\bigcup_{j=1}^{l} F_{n_{j} N} Z_{j}\right) \geq 1-\eta$.

Put $C=2 n_{l} N+1$.

Now let $G \curvearrowright(X, \mu)$ be a free p.m.p. action. Then we have $Z_{1}, \ldots, Z_{l}$ as above. Put

$$
W^{\prime}=\bigcup_{j=1}^{l} \bigcup_{x \in Z_{j}}\left(K_{n_{j} N} D \cap\left(F_{n_{j} N, x} \backslash \partial_{T} F_{n_{j} N, x}\right)\right) x
$$

and $W=\left(X \backslash S W^{\prime}\right) \cup W^{\prime}$. Then $W^{\prime}$ and $W$ are Borel subsets of $X$. Clearly $S W=X$, verifying condition (ii) in Definition 3.1 .

Let $1 \leq j \leq l$. For each $x \in Z_{j}$ we have

$$
\partial_{T} F_{n_{j} N, x} \subseteq \partial_{T} F_{n_{j} N} \cup T^{-1}\left(F_{n_{j} N} \backslash F_{n_{j} N, x}\right)
$$

and hence

$$
\begin{aligned}
\left|K_{n_{j} N} D \backslash\left(F_{n_{j} N, x} \backslash \partial_{T} F_{n_{j} N, x}\right)\right| & \leq\left|F_{n_{j} N} \backslash\left(F_{n_{j} N, x} \backslash \partial_{T} F_{n_{j} N, x}\right)\right| \\
& =\left|\left(F_{n_{j} N} \backslash F_{n_{j} N, x}\right) \cup \partial_{T} F_{n_{j} N, x}\right| \\
& \leq\left|\partial_{T} F_{n_{j} N} \cup T^{-1}\left(F_{n_{j} N} \backslash F_{n_{j} N, x}\right)\right| \\
& \leq\left|\partial_{T} F_{n_{j} N}\right|+|T| \cdot\left|F_{n_{j} N} \backslash F_{n_{j} N, x}\right| \\
& \leq 2 \eta\left|F_{n_{j} N}\right| .
\end{aligned}
$$

Set

$$
\mathcal{V}_{1}=\bigcup_{j=1}^{l} K_{n_{j} N} D Z_{j} \subseteq X, \quad \mathcal{V}_{2}=\bigcup_{j=1}^{l} D Z_{j} \subseteq X, \quad \text { and } \quad \mathcal{V}_{3}=T\left(W \backslash W^{\prime}\right) \subseteq X .
$$

Then

$$
\begin{aligned}
\Upsilon\left(S_{1}\right) \mu\left(\mathcal{V}_{1}\right) & \leq \Upsilon\left(S_{1}\right) \sum_{j=1}^{l}\left|K_{n_{j} N} D\right| \mu\left(Z_{j}\right) \\
& =\frac{\Upsilon\left(S_{1}\right)}{\left[G_{m}: G_{1}\right]} \sum_{j=1}^{l}\left|K_{n_{j} N} B D\right| \mu\left(Z_{j}\right) \\
& =\frac{\Upsilon\left(S_{1}\right)}{\left[G_{m}: G_{1}\right]} \sum_{j=1}^{l}\left|F_{n_{j} N}\right| \mu\left(Z_{j}\right) \\
& \leq \frac{\Upsilon\left(S_{1}\right)}{\left[G_{m}: G_{1}\right](1-\eta)} \leq \frac{1}{6(1-\eta)} \leq \frac{1}{3},
\end{aligned}
$$

and

$$
\Upsilon\left(S_{2}\right) \mu\left(\mathcal{V}_{2}\right) \leq \Upsilon\left(S_{2}\right) \sum_{j=1}^{l}|D| \mu\left(Z_{j}\right)
$$




$$
\begin{aligned}
& \leq \frac{\Upsilon\left(S_{2}\right)}{\left|K_{n_{0} N}\right|} \sum_{j=1}^{l}\left|K_{n_{j} N} B D\right| \mu\left(Z_{j}\right) \\
& =\frac{\Upsilon\left(S_{2}\right)}{2 n_{0} N+1} \sum_{j=1}^{l}\left|F_{n_{j} N}\right| \mu\left(Z_{j}\right) \\
& \leq \frac{\Upsilon\left(S_{2}\right)}{\left(2 n_{0} N+1\right)(1-\eta)} \leq \frac{1}{6(1-\eta)} \leq \frac{1}{3} .
\end{aligned}
$$

For each $1 \leq j \leq l$, since $K_{N} B \subseteq G_{m}$ we have

$$
F_{n_{j} N}=K_{n_{j} N} B D=A_{n_{j}-1} K_{N} B D=K_{N} B A_{n_{j}-1} D \subseteq S A_{n_{j}-1} D \subseteq S K_{n_{j} N} D .
$$

Thus $S \mathcal{V}_{1} \supseteq \bigcup_{j=1}^{l} F_{n_{j} N} Z_{j}$. Therefore

$$
\begin{aligned}
\mu\left(W \backslash W^{\prime}\right) & \leq \mu\left(X \backslash S W^{\prime}\right) \\
& \leq \mu\left(X \backslash S \mathcal{V}_{1}\right)+|S| \mu\left(\mathcal{V}_{1} \backslash W^{\prime}\right) \\
& \leq \mu\left(X \backslash \bigcup_{j=1}^{l} F_{n_{j} N} Z_{j}\right)+|S| \mu\left(\bigcup_{j=1}^{l} \bigcup_{x \in Z_{j}}\left(K_{n_{j} N} D \backslash\left(F_{n_{j} N, x} \backslash \partial_{T} F_{n_{j} N, x}\right)\right) x\right) \\
& \leq \eta+2 \eta|S| \sum_{j=1}^{l}\left|F_{n_{j} N}\right| \mu\left(Z_{j}\right) \\
& \leq \eta+\frac{2 \eta|S|}{1-\eta} \leq \eta+4 \eta|S| \leq \tau,
\end{aligned}
$$

and hence

$$
\Upsilon\left(S_{3}\right) \mu\left(\mathcal{V}_{3}\right) \leq \Upsilon(T)|T| \mu\left(W \backslash W^{\prime}\right) \leq \tau \Upsilon(T)|T| \leq \frac{1}{3}
$$

Combining estimates we obtain

$$
\sum_{j=1}^{3} \Upsilon\left(S_{j}\right) \mu\left(\mathcal{V}_{j}\right) \leq 1,
$$

verifying condition (i) in Definition 3.1.

Next let $g \in T$ and $w_{1}, w_{2} \in W$ with $g w_{1}=w_{2}$. If $w_{1} \notin W^{\prime}$ or $w_{2} \notin W^{\prime}$, then $\left(w_{1}, w_{2}\right)$ is an $S_{3}$-edge with both endpoints in $\mathcal{V}_{3}$. Thus we may assume that $w_{1}, w_{2} \in W^{\prime}$. For each $i=1,2$, we have $w_{i}=h_{i} x_{i}$ for some $1 \leq j_{i} \leq l, x_{i} \in Z_{j_{i}}$ and $h_{i} \in K_{n_{j_{i} N}} D \cap\left(F_{n_{j_{i}} N, x_{i}} \backslash \partial_{T} F_{n_{j_{i}} N, x_{i}}\right)$. Write $h_{i}$ as $t_{i} d_{i}$ for some $t_{i} \in K_{n_{j_{i}} N}$ and $d_{i} \in D$. Then $w_{i}=t_{i} d_{i} x_{i}$ is connected to $d_{i} x_{i}$ by some $S_{1}$-path in $\mathcal{V}_{1}$ of length at most $n_{l} N$. Since $h_{1} \in F_{n_{j_{1}} N, x_{1}} \backslash \partial_{T} F_{n_{j_{1}} N, x_{1}}$, we have $w_{2}=g w_{1} \in F_{n_{j_{1}} N, x_{1}} x_{1}$, and hence $x_{1}=x_{2}$. Then $\left(d_{1} x_{1}, d_{2} x_{2}\right)$ is an $S_{2}$-edge with both endpoints in $\mathcal{V}_{2}$. Thus $w_{1}$ and $w_{2}$ are connected by a path of length at most $C$ in which each edge is an $S_{j}$-edge with both endpoints in $\mathcal{V}_{j}$ for some $1 \leq j \leq 3$, verifying condition (iii) in Definition 3.1. This proves that $G$ has property SC.

To establish the shrinking property, let $\varepsilon>0$ and consider the function $\Upsilon: \mathcal{F}(G) \rightarrow[0, \infty)$ which takes the constant value $2 / \varepsilon$. Then we have $S$ as above. Take $T=\left\{e_{G}\right\}$. Let $\delta>0$. In the above we can further require that $\eta \leq \min \{\varepsilon / 2, \delta / 2\}$ and $\Upsilon\left(S_{2}\right) /\left(2 n_{0} N+1\right) \leq \delta /(2 \varepsilon)$. Given a free p.m.p. action $G \curvearrowright(X, \mu)$, we have $\mathcal{V}_{j}$ for $1 \leq j \leq 3$ as above. Put $\mathcal{V}=\mathcal{V}_{1} \cup$ 
$\left(X \backslash \bigcup_{j=1}^{l} F_{n_{j} N} Z_{j}\right)$ and $Z=\mathcal{V}_{2} \cup\left(X \backslash \bigcup_{j=1}^{l} F_{n_{j} N} Z_{j}\right) \subseteq \mathcal{V}$. From $S \mathcal{V}_{1} \supseteq \bigcup_{j=1}^{l} F_{n_{j} N} Z_{j}$ we have $S \mathcal{V}=X$, verifying condition (i) in Definition 3.6. We have

$$
\mu(\mathcal{V}) \leq \mu\left(\mathcal{V}_{1}\right)+\mu\left(X \backslash \bigcup_{j=1}^{l} F_{n_{j} N} Z_{j}\right) \leq \frac{\varepsilon}{2} \Upsilon\left(S_{1}\right) \mu\left(\mathcal{V}_{1}\right)+\eta \leq \frac{\varepsilon}{2}+\frac{\varepsilon}{2}=\varepsilon,
$$

and

$$
\mu(Z) \leq \mu\left(\mathcal{V}_{2}\right)+\mu\left(X \backslash \bigcup_{j=1}^{l} F_{n_{j} N} Z_{j}\right) \leq \frac{\varepsilon}{2} \Upsilon\left(S_{2}\right) \mu\left(\mathcal{V}_{2}\right)+\eta \leq \frac{\varepsilon \Upsilon\left(S_{2}\right)}{2 n_{0} N+1}+\frac{\delta}{2} \leq \delta,
$$

verifying condition (ii) in Definition [3.6. Clearly every point of $\mathcal{V}$ is connected to some point of $Z$ through an $S_{1}$-path of length at most $n_{l} N \leq C$ whose points all belong to $\mathcal{V}$, verifying condition (iii) in Definition 3.6. Thus $G$ has the shrinking property.

For an action $G \curvearrowright X$ and a set $F \in \mathcal{F}(G)$ we say that a subset $W$ of $X$ is the base of an $F$-tower if the sets $t W$ for $t \in F$ are pairwise disjoint.

Lemma 3.9. Let $G \curvearrowright(X, \mu)$ be a free p.m.p. action. Let $F \in \overline{\mathcal{F}}(G)$. Let $Y$ be a Borel subset of $X$ and $E$ a Borel subset of $Y^{2}$ such that $E \supseteq \Delta_{Y}=\{(y, y): y \in Y\}, E^{*}=E$ where $E^{*}:=\{(y, x):(x, y) \in E\}$, and $E$ is contained in the union of the graphs of $g \in F$. Let $M_{1} \in \mathbb{N}$. Then there are Borel sets $W, \tilde{Y} \subseteq Y$ with $\mu(Y \backslash \tilde{Y})=0$ such that

(i) $W$ is $\left(E, M_{1}\right)$-separated in the sense that no distinct two points of $W$ are connected by an E-path of length at most $2 M_{1}$, and

(ii) every point in $\tilde{Y}$ is connected to some point in $W$ by an E-path of length at most $2 M_{1}$.

Proof. Note that if a subset $U$ of $Y$ is the base of an $F^{M_{1}}$-tower, then $U$ is $\left(E, M_{1}\right)$-separated. By [24, Proposition A.22] every Borel subset $U$ of $Y$ with $\mu(U)>0$ contains a Borel subset $U^{\prime}$ with $\mu\left(U^{\prime}\right)>0$ such that $U^{\prime}$ is the base of an $F^{M_{1}}$-tower and hence is $\left(E, M_{1}\right)$-separated.

If $\mu(Y)=0$, we may take $W=\tilde{Y}=\emptyset$. Thus we may assume that $\mu(Y)>0$. By the above there are $\left(E, M_{1}\right)$-separated Borel sets $W \subseteq Y$ with $\mu(W)>0$. Denote by $\eta_{1}$ the supremum of $\mu(W)$ over all such $W$. Take one such $W_{1}$ with $\mu\left(W_{1}\right) \geq \eta_{1} / 2$.

Arguing inductively, suppose that we have chosen Borel sets $W_{1}, \ldots, W_{n} \subseteq Y$ such that $W_{1}, \ldots, W_{n}$ are pairwise disjoint and $\bigcup_{j=1}^{n} W_{j}$ is $\left(E, M_{1}\right)$-separated. Denote by $V_{n}$ the set of points in $Y$ which can be connected to some point in $\bigcup_{j=1}^{n} W_{j}$ by an $E$-path of length at most $2 M_{1}$. Then $V_{n}$ is Borel. If $\mu\left(Y \backslash V_{n}\right)=0$, we can take $W=\bigcup_{j=1}^{n} W_{j}$ and $\tilde{Y}=V_{n}$. Otherwise, by the first paragraph of the proof there are $\left(E, M_{1}\right)$-separated Borel sets $Z \subseteq Y \backslash V_{n}$ with $\mu(Z)>0$. Denote by $\eta_{n+1}$ the supremum of $\mu(Z)$ over all such $Z$. Take an $\left(E, M_{1}\right)$-separated Borel set $W_{n+1} \subseteq Y \backslash V_{n}$ such that $\mu\left(W_{n+1}\right) \geq \eta_{n+1} / 2$. Then $W_{1}, \ldots, W_{n+1}$ are pairwise disjoint and $\bigcup_{j=1}^{n+1} W_{j}$ is $\left(E, M_{1}\right)$-separated.

Now we have constructed $\left\{W_{j}\right\}_{j \in \mathbb{N}}$. Then $\mu\left(W_{j}\right) \rightarrow 0$ as $j \rightarrow \infty$ and hence $\eta_{j} \rightarrow 0$ as $j \rightarrow \infty$. Set $W=\bigcup_{j \in \mathbb{N}} W_{j} \subseteq Y$. Then $W$ is Borel and $\left(E, M_{1}\right)$-separated. Denote by $\tilde{Y}$ the set of points in $Y$ which can be connected to some point in $W$ by an $E$-path of length at most $2 M_{1}$. Then $\tilde{Y}$ is Borel. If $\mu(Y \backslash \tilde{Y})>0$, by the first paragraph of the proof there is an $\left(E, M_{1}\right)$-separated Borel set $Z \subseteq Y \backslash \tilde{Y}$ with $\mu(Z)>0$, yielding $0<\mu(Z) \leq \eta_{n}$ for all $n$, which is a contradiction. Therefore $\mu(Y \backslash \tilde{Y})=0$. 
Lemma 3.10. Suppose that $G$ is finitely generated and not virtually cyclic. Let $A$ be a generating set for $G$ in $\overline{\mathcal{F}}(G)$. Then there is a constant $b>0$ such that given any $r, M \in \mathbb{N}$ and free p.m.p. action $G \curvearrowright(X, \mu)$ there exist Borel sets $Z \subseteq \mathcal{V} \subseteq X$ such that $A^{2 r} \mathcal{V}=X, \mu(\mathcal{V}) \leq b / r$, $\mu(Z) \leq 1 /(M+1)$, and every point of $\mathcal{V}$ is connected to some point of $Z$ by an $A$-path of length at most $2 M$ with all points in $\mathcal{V}$.

Proof. As $G$ is not virtually cyclic, we can find a $c>0$ such that $\left|A^{n}\right| \geq c n^{2}$ for all $n \in \mathbb{N}$ (Corollary 3.5 of [27]). Set $b=5 / c$. Let $r, M$, and $G \curvearrowright(X, \mu)$ be as in the lemma statement.

Applying Lemma 3.9 with $F$ taken to be $A, Y$ to be $X, E$ to be the union of the graphs of $g \in A$, and $M_{1}$ to be $r$, we find a Borel set $U \subseteq X$ such that $U$ is the base of an $A^{r}$-tower and $\mu\left(A^{2 r} U\right)=1$. Set $X^{\prime}=\bigcap_{g \in G} g\left(A^{2 r} U\right)$. Then $X^{\prime}$ is $G$-invariant and $\mu\left(X^{\prime}\right)=1$. Set $U^{\prime}=U \cap X^{\prime}$. Then $U^{\prime}$ is Borel. For each $x \in X^{\prime}$, we have $x \in A^{2 r} w$ for some $w \in U$. Then $w \in A^{2 r} x \subseteq X^{\prime}$ and hence $w \in U^{\prime}$. This shows that $X^{\prime}=A^{2 r} U^{\prime}$. Note also that

$$
\left|A^{r}\right| \mu\left(U^{\prime}\right)=\mu\left(A^{r} U^{\prime}\right) \leq 1
$$

whence

$$
\mu\left(U^{\prime}\right) \leq \frac{1}{\left|A^{r}\right|} \leq \frac{1}{c r^{2}}
$$

Let $w \in U^{\prime}$. Set $T_{w}=U^{\prime} \cap G w$. Since $X^{\prime}=A^{2 r} U^{\prime}$, we have $G w=A^{2 r} T_{w}$. In particular, $T_{w}$ is infinite. Consider the graph $\left(T_{w}, E_{w}^{\prime}\right)$ whose edges are those pairs of vertices which can be joined by an $A$-path of length at most $4 r+1$. We claim that $\left(T_{w}, E_{w}^{\prime}\right)$ is connected. It suffices to show that every $v \in T_{w}$ is connected to $w$ by some path in $\left(T_{w}, E_{w}^{\prime}\right)$. Take an $A$-path from $w$ to $v$. For each point $z$ in this path, we may connect $z$ to some $u_{z} \in T_{w}$ by an $A$-path $p_{z}$ of length at most $2 r$. Inserting $p_{z}$ and the reverse of $p_{z}$ at $z$, we find an $A$-path from $w$ to $v$ in which points of $T_{w}$ appear in every interval of length $4 r+1$. Thus $v$ is connected to $w$ by some path in $\left(T_{w}, E_{w}^{\prime}\right)$. This proves our claim.

Consider the graph $\left(U^{\prime}, E^{\prime}\right)$ whose edges are those pairs of vertices which can be joined by an $A$-path of length at most $4 r+1$. Note that $E^{\prime}$ is a Borel subset of $\left(U^{\prime}\right)^{2}$ and is contained in the union of the graphs of $g \in A^{4 r+1}$.

Applying Lemma 3.9 with $F=A^{4 r+1}, Y=U^{\prime}, E=E^{\prime}$, and $M_{1}=M$, we find an $\left(E^{\prime}, M\right)$ separated Borel subset $Z^{\prime}$ of $U^{\prime}$ such that $\mu\left(U^{\prime} \backslash W^{\prime}\right)=0$, where $W^{\prime}$ denotes the Borel set of all points in $U^{\prime}$ which can be connected to some point in $Z^{\prime}$ by an $E^{\prime}$-path of length at most $2 M$. For each $0 \leq j \leq 2 M$, denote by $W_{j}^{\prime}$ the set of points in $W^{\prime}$ which can be connected to some point in $Z^{\prime}$ by an $E^{\prime}$-path of length $j$, but cannot be connected to any point in $Z^{\prime}$ by an $E^{\prime}$-path of length less than $j$. Then the sets $Z^{\prime}=W_{0}^{\prime}, W_{1}^{\prime}, W_{2}^{\prime}, \ldots, W_{2 M}^{\prime}$ form a Borel partition of $W^{\prime}$.

Denote by $\Theta$ the set of finite sequences in $A$ with length at most $4 r+1$. Let $1 \leq j \leq 2 M$. Take a Borel map $f_{j}: W_{j}^{\prime} \rightarrow W_{j-1}^{\prime}$ such that $\left(v, f_{j}(v)\right) \in E^{\prime}$ for all $v \in W_{j}^{\prime}$. Also take a Borel map $h_{j}: W_{j}^{\prime} \rightarrow \Theta$ such that for any $v \in W_{j}^{\prime}$, say $h_{j}(v)=\left(g_{1}, \ldots, g_{l}\right)$ with $1 \leq l \leq 4 r+1$ and $g_{k} \in A$ for $1 \leq k \leq l$, one has $g_{l} g_{l-1} \ldots g_{1} v=f_{j}(v)$. Denote by $\mathcal{V}^{\prime}$ the union of $W^{\prime}$ and the set consisting of the points $g_{1} v, g_{2} g_{1} v, \ldots, g_{l-1} \ldots g_{1} v$ for all $1 \leq j \leq 2 M, v \in W_{j}^{\prime}$, and $h_{j}(v)=\left(g_{1}, \ldots, g_{l}\right)$. Also, denote by $\mathcal{E}$ the union of $\Delta_{\mathcal{V}^{\prime}}$ and the set consisting of all pairs of the form $\left(g_{k} g_{k-1} \ldots g_{1} v, g_{k-1} \ldots g_{1} v\right)$ or $\left(g_{k-1} \ldots g_{1} v, g_{k} g_{k-1} \ldots g_{1} v\right)$ for $1 \leq j \leq 2 M, v \in W_{j}^{\prime}$, 
$h_{j}(v)=\left(g_{1}, \ldots, g_{l}\right)$, and $1 \leq k \leq l$. Then $\mathcal{V}^{\prime}$ is Borel, and

$$
\mu\left(\mathcal{V}^{\prime}\right) \leq \sum_{j=0}^{2 M}(4 r+1) \mu\left(W_{j}^{\prime}\right)=(4 r+1) \mu\left(W^{\prime}\right) \leq 5 r \mu\left(W^{\prime}\right)=5 r \mu\left(U^{\prime}\right) \stackrel{\text { (1) }}{\leq} 5 r \cdot \frac{1}{c r^{2}}=\frac{b}{r} .
$$

Note that $\mathcal{E} \subseteq\left(\mathcal{V}^{\prime}\right)^{2}$ is Borel and contained in the union of the graphs of $g \in A$, and that $\mathcal{E} \supseteq \Delta_{\mathcal{V}^{\prime}}$ and $\mathcal{E}^{*}=\mathcal{E}$. Also note that every point of $\mathcal{V}^{\prime}$ is connected to some point of $Z^{\prime}$ by an $\mathcal{E}$-path of length at most $2 M(4 r+1)$.

Let $z \in Z^{\prime}$. Denote by $V_{z}^{\prime}$ the set of all $w \in U^{\prime}$ which can be connected to $z$ by an $E^{\prime}$-path of length at most $M$. Since $T_{z}$ is infinite, $T_{z} \neq V_{z}^{\prime}$. Because $\left(T_{z}, E_{z}^{\prime}\right)$ is connected, we conclude that $\left|V_{z}^{\prime}\right| \geq M+1$. Since $Z^{\prime}$ is $\left(E^{\prime}, M\right)$-separated, the sets $V_{z}^{\prime}$ for $z \in Z^{\prime}$ are pairwise disjoint, and hence for each $z \in Z^{\prime}$ the set $V_{z}^{\prime}$ is contained in the $\mathcal{E}$-connected component of $\mathcal{V}^{\prime}$ containing $z$. As every $\mathcal{E}$-connected component of $\mathcal{V}^{\prime}$ contains some $z \in Z^{\prime}$, it contains $V_{z}^{\prime}$ and hence has at least $M+1$ points.

Applying Lemma 3.9 with $F=A, Y=\mathcal{V}^{\prime}, E=\mathcal{E}$, and $M_{1}=M$, we find an $(\mathcal{E}, M)$-separated Borel subset $Z$ of $\mathcal{V}^{\prime}$ such that $\mu\left(\mathcal{V}^{\prime} \backslash \mathcal{V}\right)=0$, where $\mathcal{V}$ denotes the Borel set of all points in $\mathcal{V}^{\prime}$ which can be connected to some point in $Z$ by an $\mathcal{E}$-path of length at most $2 M$. Then every point in $\mathcal{V}$ can be connected to some point of $Z$ by an $A$-path of length at most $2 M$ with all points in $\mathcal{V}$. Note that $\mu(\mathcal{V})=\mu\left(\mathcal{V}^{\prime}\right) \leq b / r$.

Let $z \in Z$. Denote by $V_{z}$ the set of $w \in \mathcal{V}^{\prime}$ which can be connected to $z$ by an $\mathcal{E}$-path of length at most $M$. Since the $\mathcal{E}$-connected component of $\mathcal{V}^{\prime}$ containing $z$ has at least $M+1$ points, we have $\left|V_{z}\right| \geq M+1$. Note that $V_{z}=U_{z} z$ for some subset $U_{z}$ of $A^{M}$. Denote by $\mathcal{D}$ the set of all subsets of $A^{M}$ with cardinality at least $M+1$. The map $\psi$ from $Z$ to $\mathcal{D}$ sending $z$ to $U_{z}$ is Borel. Since the sets $V_{z}$ for $z \in Z$ are pairwise disjoint, we get

$$
\begin{aligned}
1 \geq \mu\left(\bigcup_{z \in Z} V_{z}\right)=\mu\left(\bigcup_{z \in Z} U_{z} z\right) & =\sum_{D \in \mathcal{D}} \mu\left(\bigcup_{z \in \psi^{-1}(D)} D z\right) \\
& =\sum_{D \in \mathcal{D}}|D| \mu\left(\psi^{-1}(D)\right) \\
& \geq(M+1) \sum_{D \in \mathcal{D}} \mu\left(\psi^{-1}(D)\right)=(M+1) \mu(Z),
\end{aligned}
$$

whence

$$
\mu(Z) \leq \frac{1}{M+1}
$$

Since $\mu\left(\mathcal{V}^{\prime} \backslash \mathcal{V}\right)=0$, we have

$$
\mu\left(A^{2 r} \mathcal{V}\right)=\mu\left(A^{2 r} \mathcal{V}^{\prime}\right) \geq \mu\left(A^{2 r} W^{\prime}\right)=\mu\left(A^{2 r} U^{\prime}\right)=\mu\left(X^{\prime}\right)=1,
$$

and hence $\mu\left(A^{2 r} \mathcal{V}\right)=1$.

Now put $\mathcal{V}^{b}=\mathcal{V} \cup\left(X \backslash A^{2 r} \mathcal{V}\right)$ and $Z^{b}=Z \cup\left(X \backslash A^{2 r} \mathcal{V}\right)$. Then $Z^{b} \subseteq \mathcal{V}^{b}, A^{2 r} \mathcal{V}^{b}=X$, $\mu\left(\mathcal{V}^{b}\right)=\mu(\mathcal{V}) \leq b / r, \mu\left(Z^{b}\right)=\mu(Z) \leq 1 /(M+1)$, and every point of $\mathcal{V}^{b}$ is connected to some point of $Z^{b}$ by an $A$-path of length at most $2 M$ with all points in $\mathcal{V}^{b}$.

Proposition 3.11. G has the shrinking property if and only if it is neither locally finite nor virtually cyclic. 
Proof. If $G$ is not locally virtually cyclic, then $G$ has a finitely generated subgroup $G_{1}$ which is not virtually cyclic. By Lemma 3.10 the group $G_{1}$ has the shrinking property, and hence so does $G$. If $G$ is locally virtually cyclic but neither locally finite nor virtually cyclic, then by Lemma 3.8 the group $G$ has the shrinking property. This proves the "if" part.

To prove the "only if" part, we assume that $G$ has the shrinking property and argue by contradiction that $G$ cannot be locally finite or virtually cyclic.

Suppose that $G$ is locally finite. Let $S_{1} \in \overline{\mathcal{F}}(G)$ witness the shrinking property for $G$. Denote by $G_{1}$ the finite subgroup of $G$ generated by $S_{1}$. Put $\varepsilon=1$. Then we have $S \in \overline{\mathcal{F}}(G)$ in Definition [3.6. Take $0<\delta<1 /\left|S G_{1}\right|$. Then we have $C$ in Definition 3.6, Let $G \curvearrowright(X, \mu)$ be a free p.m.p. action. Then we have Borel sets $Z \subseteq \mathcal{V} \subseteq X$ satisfying conditions (i)-(iii) in Definition [3.6. Note that $\mathcal{V} \subseteq G_{1} Z$ and hence $X=S \mathcal{V} \subseteq S G_{1} Z$. Thus $\delta \geq \mu(Z) \geq 1 /\left|S G_{1}\right|$, which is a contradiction.

Suppose now that $G$ is virtually cyclic but not locally finite. Then $G$ has a finite-index normal subgroup $G_{1}$ isomorphic to $\mathbb{Z}$. Take a generator $s$ for $G_{1}$. For each $n \in \mathbb{N}$ put $K_{n}:=\left\{s^{j}:-n \leq\right.$ $j \leq n\}$. Take a $B \in \mathcal{F}(G)$ containing $e_{G}$ such that $G=\bigsqcup_{b \in B} G_{1} b$. Let $S_{1} \in \overline{\mathcal{F}}(G)$ witness the shrinking property for $G$. Take $m \in \mathbb{N}$ such that $B S_{1} \subseteq K_{m} B$. Take $0<\varepsilon<1 /\left(8\left|K_{m} B\right|\right)$. Then we have $S \in \overline{\mathcal{F}}(G)$ in Definition [3.6. Take an $S^{\prime} \in \overline{\mathcal{F}}\left(G_{1}\right)$ such that $S^{\prime} B \supseteq S$. Then $S^{\prime} \subseteq K_{M}$ for some $M \in \mathbb{N}$. Take $R \in \mathbb{N}$ large enough so that $(2 R+2 M+1) /(2 R+1) \leq 2$ and take $0<\delta<1 /\left(2\left|S^{\prime} K_{R} B\right|\right)$. Then we have $C \in \mathbb{N}$ in Definition 3.6. Let $G \curvearrowright(X, \mu)$ be a free p.m.p. action. Then we have Borel sets $Z \subseteq \mathcal{V} \subseteq X$ satisfying conditions (i)-(iii) in Definition 3.6. Put $\mathcal{V}^{\prime}=B \mathcal{V}, \mathcal{V}^{\prime \prime}=K_{m} \mathcal{V}^{\prime}$, and $Z^{\prime}=B Z \subseteq \mathcal{V}^{\prime}$. Then $X=S \mathcal{V} \subseteq S^{\prime} B \mathcal{V}=S^{\prime} \mathcal{V}^{\prime}$ and hence $X=S^{\prime} \mathcal{V}^{\prime}$.

We claim that every point of $\mathcal{V}^{\prime}$ can be connected to some point of $Z^{\prime}$ by a $K_{1}$-path whose points all belong to $\mathcal{V}^{\prime \prime}$. Let $w^{\prime} \in \mathcal{V}^{\prime}$. Then $w^{\prime}=b w$ for some $w \in \mathcal{V}$ and $b \in B$. We can find some $1 \leq l \leq C$ and $g_{1}, \ldots, g_{l} \in S_{1}$ such that $g_{j} \ldots g_{1} w \in \mathcal{V}$ for all $1 \leq j \leq l$ and $g_{l} \ldots g_{1} w \in Z$. Put $b_{0}=b$. We define $b_{j} \in B$ and $h_{j} \in K_{m}$ for $1 \leq j \leq l$ inductively by $b_{j-1} g_{j}^{-1}=h_{j}^{-1} b_{j}$. For each $1 \leq j \leq l$, we have

$$
b_{j}^{-1} h_{j} \ldots h_{1} b w=g_{j} b_{j-1}^{-1} h_{j-1} \ldots h_{1} b w=\cdots=g_{j} g_{j-1} \ldots g_{1} w \in \mathcal{V},
$$

and hence $h_{j} \ldots h_{1} b w \in b_{j} \mathcal{V} \subseteq \mathcal{V}^{\prime}$. Furthermore, $h_{l} \ldots h_{1} b w=b_{l} g_{l} \ldots g_{1} w \in b_{l} Z \subseteq Z^{\prime}$. We can connect $h_{j} \ldots h_{1} b w$ and $h_{j-1} \ldots h_{1} b w$ by a $K_{1}$-path of length at most $m$ with all points in $\mathcal{V}^{\prime \prime}$. This proves our claim.

Put $U=X \backslash S^{\prime} K_{R} Z^{\prime}$. Let $u \in U$. Since $X=S^{\prime} \mathcal{V}^{\prime}$, we have $g u=w^{\prime}$ for some $g \in S^{\prime} \subseteq K_{M}$ and $w^{\prime} \in \mathcal{V}^{\prime}$. Then $w^{\prime} \notin K_{R} Z^{\prime}$. By the claim above we can find a $K_{1}$-path in $\mathcal{V}^{\prime \prime}$ from $w^{\prime}$ to some point $z^{\prime} \in Z^{\prime}$. It follows that either $s^{j} w^{\prime} \in \mathcal{V}^{\prime \prime}$ for all $0 \leq j \leq R$ or $s^{-j} w^{\prime} \in \mathcal{V}^{\prime \prime}$ for all $0 \leq j \leq R$. Therefore

$$
\frac{1}{2 R+1} \sum_{j=-R-M}^{R+M} \chi_{\mathcal{V}^{\prime \prime}}\left(s^{j} u\right) \geq \frac{1}{2 R+1} \sum_{j=-R}^{R} \chi_{\mathcal{V}^{\prime \prime}}\left(s^{j} w^{\prime}\right) \geq \frac{1}{2} .
$$

Thus

$$
\begin{aligned}
\int_{U} \frac{1}{2 R+1} \sum_{j=-R-M}^{R+M} \chi \mathcal{V}^{\prime \prime}\left(s^{j} u\right) d \mu(u) & \geq \int_{U} \frac{1}{2} d \mu(u) \\
& =\frac{1}{2} \mu(U)
\end{aligned}
$$




$$
\begin{aligned}
& \geq \frac{1}{2}\left(1-\left|S^{\prime} K_{R} B\right| \mu(Z)\right) \\
& \geq \frac{1}{2}\left(1-\left|S^{\prime} K_{R} B\right| \delta\right)>\frac{1}{4},
\end{aligned}
$$

while

$$
\begin{aligned}
\int_{U} \frac{1}{2 R+1} \sum_{j=-R-M}^{R+M} \chi \mathcal{V}^{\prime \prime}\left(s^{j} u\right) d \mu(u) & \leq \int_{X} \frac{1}{2 R+1} \sum_{j=-R-M}^{R+M} \chi \mathcal{V}^{\prime \prime}\left(s^{j} u\right) d \mu(u) \\
& =\frac{2 R+2 M+1}{2 R+1} \cdot \mu\left(\mathcal{V}^{\prime \prime}\right) \\
& \leq 2\left|K_{m} B\right| \mu(\mathcal{V}) \\
& \leq 2 \varepsilon\left|K_{m} B\right|<\frac{1}{4}
\end{aligned}
$$

a contradiction. This proves the "only if" part.

3.3. Variants of property SC. For the purposes of Section 3.5 it will be convenient to formulate the following variations on property SC. Proposition 3.15 establishes relationships between these two properties, property SC itself, and the shrinking property.

Definition 3.12. Let $\mathfrak{Y}$ be a class of free p.m.p. actions of a fixed $G$. We say that $\mathfrak{Y}$ has property $S C^{\prime}$ if for any function $\Upsilon: \mathcal{F}(G) \rightarrow[0, \infty)$ there is some $\eta>0$ such that for any $T \in \overline{\mathcal{F}}(G)$ there are $C, n \in \mathbb{N}$, and $S_{1}, \ldots, S_{n} \in \overline{\mathcal{F}}(G)$ so that for any $G \curvearrowright(X, \mu)$ in $\mathfrak{Y}$ and any Borel sets $W_{1}, W_{2} \subseteq X$ with $\mu\left(W_{1}\right), \mu\left(W_{2}\right) \leq \eta$, there are Borel sets $\mathcal{V}_{1}, \ldots, \mathcal{V}_{n} \subseteq X$ satisfying the following conditions:

(i) $\sum_{j=1}^{n} \Upsilon\left(S_{j}\right) \mu\left(\mathcal{V}_{j}\right) \leq 1$,

(ii) if $w_{1} \in W_{1}$ and $w_{2} \in W_{2}$ satisfy $g w_{1}=w_{2}$ for some $g \in T$ then $w_{1}$ and $w_{2}$ are connected by a path of length at most $C$ in which each edge is an $S_{j}$-edge with both endpoints in $\mathcal{V}_{j}$ for some $1 \leq j \leq n$.

We say that $\mathfrak{Y}$ has property $S C^{\prime \prime}$ if the above conditions hold without the bound $C$.

Lemma 3.13. Suppose that $G$ is locally finite. Then no free p.m.p. action $G \curvearrowright(X, \mu)$ has property $S C^{\prime \prime}$.

Proof. Let $G \curvearrowright(X, \mu)$ be a free p.m.p. action. Take a strictly increasing sequence $\left\{G_{k}\right\}_{k \in \mathbb{N}}$ of finite subgroups of $G$ such that $G=\bigcup_{k \in \mathbb{N}} G_{k}$. For each $F \in \mathcal{F}(G)$, denote by $\Phi(F)$ the smallest $k \in \mathbb{N}$ satisfying $F \subseteq G_{k}$. Define $\Upsilon: \mathcal{F}(G) \rightarrow[0, \infty)$ by $\Upsilon(F)=2\left|G_{\Phi(F)}\right|$.

Suppose that $G \curvearrowright(X, \mu)$ has property $\mathrm{SC}^{\prime \prime}$. Then there is an $\eta>0$ satisfying the conditions in Definition 3.12. Take an $m \in \mathbb{N}$ with $1 /\left|G_{m}\right|<\eta$. Put $T=G_{m+1} \in \overline{\mathcal{F}}(G)$. Then there are $n \in \mathbb{N}$ and $S_{1}, \ldots, S_{n} \in \overline{\mathcal{F}}(G)$ satisfying the conditions in Definition 3.12, We may assume that there is a $k$ such that $S_{1}, \ldots, S_{k} \subseteq G_{m}$ and none of $S_{k+1}, \ldots, S_{n}$ is contained in $G_{m}$.

As $G_{m+1}$ is finite we can find a Borel set $Y \subseteq X$ such that the sets $g Y$ for $g \in G_{m+1}$ form a partition of $X$ (see Example 6.1 and Proposition 6.4 in [21]). Choose an $h \in G_{m+1} \backslash G_{m}$. Put $W_{1}=Y$ and $W_{2}=h Y$, and note that $\mu\left(W_{1}\right)=\mu\left(W_{2}\right)=1 /\left|G_{m+1}\right|<\eta$. Then there are Borel sets $\mathcal{V}_{1}, \ldots, \mathcal{V}_{n} \subseteq X$ satisfying the conditions in Definition 3.12 , 
Let $y \in Y$. By condition (ii) in Definition 3.12, $y$ and hy are connected by a path each of whose edges is an $S_{j}$-edge with both endpoints in $\mathcal{V}_{j}$ for some $1 \leq j \leq n$. Since $h \notin G_{m}$, this implies that $G_{m} y \cap \bigcup_{j=k+1}^{n} \mathcal{V}_{j} \neq \emptyset$. We infer that $\mu\left(\bigcup_{j=k+1}^{n} \mathcal{V}_{j}\right) \geq \mu(Y)=1 /\left|G_{m+1}\right|$ and hence

$$
\begin{aligned}
1 \geq \sum_{j=1}^{n} \Upsilon\left(S_{j}\right) \mu\left(\mathcal{V}_{j}\right) & \geq \sum_{j=k+1}^{n} \Upsilon\left(S_{j}\right) \mu\left(\mathcal{V}_{j}\right) \\
& \geq 2\left|G_{m+1}\right| \sum_{j=k+1}^{n} \mu\left(\mathcal{V}_{j}\right) \geq 2\left|G_{m+1}\right| \mu\left(\bigcup_{j=k+1}^{n} \mathcal{V}_{j}\right) \geq 2,
\end{aligned}
$$

a contradiction. We conclude that $G \curvearrowright(X, \mu)$ does not have property $\mathrm{SC}^{\prime \prime}$.

Lemma 3.14. Suppose that $G$ is virtually cyclic. Then no free p.m.p. action $G \curvearrowright(X, \mu)$ has property $S C^{\prime \prime}$.

Proof. By assumption $G$ has a subgroup of finite index isomorphic to $\mathbb{Z}$. Then $G$ has a normal subgroup $G_{1}$ of finite index isomorphic to $\mathbb{Z}$. Take a generator $s$ for $G_{1}$. Take a finite subset $B$ of $G$ containing $e_{G}$ such that $G$ is the disjoint union of the sets $h G_{1}$ for $h \in B$. For each $m \in \mathbb{N}$ put $K_{m}=\left\{s^{k}:-m \leq k \leq m\right\}$. For each $F \in \mathcal{F}(G)$, denote by $\Phi(F)$ the smallest $m \in \mathbb{N}$ satisfying $F \subseteq B K_{m}$. Put $M=\Phi(B B)$. Define $\Upsilon: \mathcal{F}(G) \rightarrow[0, \infty)$ by $\Upsilon(F)=3\left|K_{M}\right| \cdot|B| \cdot\left|B K_{\Phi(F)}\right|$.

Let $G \curvearrowright(X, \mu)$ be a free p.m.p. action and suppose that it has property $\mathrm{SC}^{\prime \prime}$. Then there is an $\eta>0$ satisfying the conditions in Definition 3.12. Take an $m \in \mathbb{N}$ with $1 /(m+1)<\eta$. Put $T=\left\{s^{m}, e_{G}, s^{-m}\right\} \in \overline{\mathcal{F}}(G)$. Then there are $n \in \mathbb{N}$ and $S_{1}, \ldots, S_{n} \in \overline{\mathcal{F}}(G)$ satisfying the conditions in Definition 3.12 ,

Applying the Rokhlin lemma [24, Lemma 4.77] to $G_{1} \curvearrowright(X, \mu)$, we find a Borel set $Y \subseteq X$ such that the sets $s^{k} Y$ for $k=0,1, \ldots, m$ are pairwise disjoint and $\mu\left(\bigcup_{k=0}^{m} s^{k} Y\right)>1 / 2$. Put $W_{1}=Y$ and $W_{2}=s^{m} Y$. We have $\mu\left(W_{1}\right)=\mu\left(W_{2}\right)=\mu(Y) \leq 1 /(m+1)<\eta$. Then there are Borel sets $\mathcal{V}_{1}, \ldots, \mathcal{V}_{n} \subseteq X$ satisfying the conditions in Definition 3.12 .

Put $\mathcal{V}=\bigcup_{j=1}^{n} B K_{\Phi\left(S_{j}\right)} \mathcal{V}_{j}$. If $\left(v_{1}, v_{2}\right)$ is an $S_{j}$-edge with both $v_{1}$ and $v_{2}$ in $\mathcal{V}_{j}$ for some $1 \leq j \leq n$, then $v_{1}$ and $v_{2}$ are connected by a $\left(B \cup K_{1}\right)$-path in $\mathcal{V}$. From condition (ii) in Definition 3.12 we conclude that for each $y \in Y$ the points $y$ and $s^{m} y$ are connected by a $\left(B \cup K_{1}\right)$-path in $\mathcal{V}$. Put $\mathcal{V}^{\prime}=B^{-1} \mathcal{V}$ and $\mathcal{V}^{\prime \prime}=K_{M} \mathcal{V}^{\prime}$.

Let $x \in \mathcal{V}^{\prime}, h \in B$, and $l \in \mathbb{N}$ be such that $s^{k} h x \in \mathcal{V}$ for all $0 \leq k \leq l$. Then either $h^{-1} s^{i} h=s^{i}$ for all $i \in \mathbb{Z}$ or $h^{-1} s^{i} h=s^{-i}$ for all $i \in \mathbb{Z}$. If $h^{-1} s^{i} h=s^{i}$ for all $i \in \mathbb{Z}$, then $x$ is connected to $s^{l} h x$ by the path $x, s x, \ldots, s^{l} x, h s^{l} x$, which is the $K_{1}$-path $x, s x, \ldots, s^{l} x$ in $\mathcal{V}^{\prime}$ followed by the $B$-edge $\left(s^{l} x, h s^{l} x\right)$ with $s^{l} x \in \mathcal{V}^{\prime}$ and $h s^{l} x \in \mathcal{V}$, since $s^{k} x=h^{-1} s^{k} h x \in \mathcal{V}^{\prime}$ for all $0 \leq k \leq l$. If $h^{-1} s^{i} h=s^{-i}$ for all $i \in \mathbb{Z}$, then $x$ is connected to $s^{l} h x$ by the path $x, s^{-1} x, \ldots, s^{-l} x, h s^{-l} x$, which is the $K_{1}$-path $x, s^{-1} x, \ldots, s^{-l} x$ in $\mathcal{V}^{\prime}$ followed by the $B$-edge $\left(s^{-l} x, h s^{-l} x\right)$ with $s^{-l} x \in \mathcal{V}^{\prime}$ and $h s^{-l} x \in \mathcal{V}$, since $s^{-k} x=h^{-1} s^{k} h x \in \mathcal{V}^{\prime}$ for all $0 \leq k \leq l$.

Similarly, if $x \in \mathcal{V}^{\prime}, h \in B$, and $l \in \mathbb{N}$ are such that $s^{-k} h x \in \mathcal{V}$ for all $0 \leq k \leq l$ then $x$ is connected to $s^{-l} h x$ by a path which is a $K_{1}$-path in $\mathcal{V}^{\prime}$ followed by a $B$-edge of the form $\left(z, s^{-l} h x\right)$ with $z \in \mathcal{V}^{\prime}$ and $s^{-l} h x \in \mathcal{V}$.

Let $x \in \mathcal{V}^{\prime}$ and $h_{1}, h_{2} \in B$ be such that $h_{2} h_{1} x, h_{1} x \in \mathcal{V}$. Then $h_{2} h_{1}=h s^{k}$ for some $h \in B$ and $-M \leq k \leq M$. When $k \geq 0, x$ is connected to $h_{2} h_{1} x$ by the path $x, s x, \ldots, s^{k} x, h s^{k} x$, which is the $K_{1}$-path $x, s x, \ldots, s^{k} x$ in $\mathcal{V}^{\prime \prime}$ followed by the $B$-edge $\left(s^{k} x, h s^{k} x\right)$ with $s^{k} x \in \mathcal{V}^{\prime}$ and 
$h s^{k} x \in \mathcal{V}$. When $k \leq 0, x$ is connected to $h_{2} h_{1} x$ by the path $x, s^{-1} x, \ldots, s^{k} x, h s^{k} x$, which is the $K_{1}$-path $x, s^{-1} x, \ldots, s^{k} x$ in $\mathcal{V}^{\prime \prime}$ followed by the $B$-edge $\left(s^{k} x, h s^{k} x\right)$ with $s^{k} x \in \mathcal{V}^{\prime}$ and $h s^{k} x \in \mathcal{V}$.

From the above three paragraphs, we conclude that if $x \in \mathcal{V}$ is connected to $y \in \mathcal{V}$ by a $\left(B \cup K_{1}\right)$-path in $\mathcal{V}$, then $x$ is connected to $y$ by a path in $\mathcal{V}^{\prime \prime}$ that is a $K_{1}$-path followed by a $B$-edge.

For any $y \in Y$, since $y$ and $s^{m} y$ are connected by a $\left(B \cup K_{1}\right)$-path in $\mathcal{V}$ we infer that $y$ is connected to $s^{m} y$ by a $K_{1}$-path in $\mathcal{V}^{\prime \prime}$. Therefore $s^{k} y \in \mathcal{V}^{\prime \prime}$ for all $0 \leq k \leq m$. Consequently, $\mu\left(\mathcal{V}^{\prime \prime}\right) \geq \mu\left(\bigcup_{k=0}^{m} s^{k} Y\right) \geq 1 / 2$. On the other hand, we have

$$
\mu\left(\mathcal{V}^{\prime \prime}\right) \leq\left|K_{M}\right| \cdot|B| \mu(\mathcal{V}) \leq \sum_{j=1}^{n}\left|K_{M}\right| \cdot|B| \cdot\left|B K_{\Phi\left(S_{j}\right)}\right| \mu\left(\mathcal{V}_{j}\right)=\frac{1}{3} \sum_{j=1}^{n} \Upsilon\left(S_{j}\right) \mu\left(\mathcal{V}_{j}\right) \leq \frac{1}{3},
$$

a contradiction. We conclude that $G \curvearrowright(X, \mu)$ does not have property $\mathrm{SC}^{\prime \prime}$.

Proposition 3.15. For a fixed $G$, let $\mathfrak{Y}$ be a nonempty class of free p.m.p. actions $G \curvearrowright(X, \mu)$. Consider the following conditions:

(i) $\mathfrak{Y}$ has property $S C$,

(ii) $\mathfrak{Y}$ has property $S C^{\prime}$,

(iii) $\mathfrak{Y}$ has property $S C^{\prime \prime}$,

(iv) $G$ has the shrinking property.

Then $(i) \Leftrightarrow(i i) \Rightarrow($ iii $) \Rightarrow($ iv $)$. Moreover, if $\mathfrak{Y}$ consists of a single action or of all free p.m.p. actions of $G$ then $(i) \Leftrightarrow(i i) \Leftrightarrow(i i i) \Rightarrow(i v)$.

Proof. (i) $\Rightarrow$ (ii). Let $\Upsilon$ be a function $\mathcal{F}(G) \rightarrow[0, \infty)$. Then we have an $S \in \overline{\mathcal{F}}(G)$ witnessing property SC for $\mathfrak{Y}$ with respect to the function $2 \Upsilon$. Take an $\eta>0$ with $2 \eta|S| \Upsilon(S)<1 / 2$. Let $T_{1} \in \overline{\mathcal{F}}(G)$. Then we have $C, n, S_{1}, \ldots, S_{n}$ given by Definition 3.1 for $T:=S T_{1} S \in \overline{\mathcal{F}}(G)$. Put $S_{n+1}=S \in \overline{\mathcal{F}}(G)$. Let $G \curvearrowright(X, \mu)$ be an action in $\mathfrak{Y}$. Then we have $W, \mathcal{V}_{1}, \ldots, \mathcal{V}_{n}$ satisfying conditions (i)-(iii) in Definition 3.1. Let $W_{1}, W_{2}$ be Borel subsets of $X$ satisfying $\mu\left(W_{1}\right), \mu\left(W_{2}\right) \leq \eta$. Put

$$
\mathcal{V}_{n+1}=\left(S_{n+1}\left(W_{1} \cup W_{2}\right) \cap W\right) \cup\left(W_{1} \cup W_{2}\right) \subseteq S\left(W_{1} \cup W_{2}\right) .
$$

Then

and hence

$$
\mu\left(\mathcal{V}_{n+1}\right) \leq|S|\left(\mu\left(W_{1}\right)+\mu\left(W_{2}\right)\right) \leq 2 \eta|S|
$$

$$
\sum_{j=1}^{n+1} \Upsilon\left(S_{j}\right) \mu\left(\mathcal{V}_{j}\right) \leq \frac{1}{2}+\Upsilon(S) \mu\left(\mathcal{V}_{n+1}\right) \leq \frac{1}{2}+2 \eta|S| \Upsilon(S) \leq \frac{1}{2}+\frac{1}{2}=1,
$$

verifying condition (i) in Definition 3.12, Let $g \in T_{1}$ and $w_{1} \in W_{1}, w_{2} \in W_{2}$ with $g w_{1}=w_{2}$. For $i=1,2$ take an $s_{i} \in S$ such that $s_{i} w_{i} \in W$. Then $\left(w_{i}, s_{i} w_{i}\right)$ is an $S_{n+1}$-edge with both endpoints in $\mathcal{V}_{n+1}$. Note that $\left(s_{2} g s_{1}^{-1}\right)\left(s_{1} w_{1}\right)=s_{2} w_{2}$ and $s_{2} g s_{1}^{-1} \in T$. Thus $s_{1} w_{1}$ and $s_{2} w_{2}$ are connected by a path of length at most $C$ in which each edge is an $S_{j}$-edge with both endpoints in $\mathcal{V}_{j}$ for some $1 \leq j \leq n$. Then $w_{1}$ and $w_{2}$ are connected by a path of length at most $C+2$ in which each edge is an $S_{j}$-edge with both endpoints in $\mathcal{V}_{j}$ for some $1 \leq j \leq n+1$, verifying condition (ii) in Definition 3.12, Thus $\mathfrak{Y}$ has property $\mathrm{SC}^{\prime}$.

(ii) $\Rightarrow$ (iii). Trivial.

(iii) $\Rightarrow$ (iv). This follows from Lemmas 3.13 and 3.14 and Proposition 3.11 . 
(ii) $\Rightarrow$ (i). Since (ii) $\Rightarrow($ iii $) \Rightarrow($ iv $), G$ has the shrinking property. Let $S^{\sharp} \in \overline{\mathcal{F}}(G)$ witness the shrinking property for $G$. Let $\Upsilon$ be a function $\mathcal{F}(G) \rightarrow[0, \infty)$. Take an $\eta$ as given by property $\mathrm{SC}^{\prime}$ for $\mathfrak{Y}$ with respect to the function $2 \Upsilon$. Take an $\varepsilon^{\sharp}>0$ with $\varepsilon^{\sharp} \Upsilon\left(S^{\sharp}\right) \leq 1 / 2$. By the shrinking property for $G$ there is an $S \in \overline{\mathcal{F}}(G)$ so that for any $\delta>0$ there is a $C^{\sharp} \in \mathbb{N}$ such that given any free p.m.p. action $G \curvearrowright(X, \mu)$ we can find Borel sets $Z \subseteq \mathcal{V} \subseteq X$ satisfying the following conditions:

(a) $S \mathcal{V}=X$,

(b) $\mu(\mathcal{V}) \leq \varepsilon^{\sharp}$ and $\mu(Z) \leq \delta$,

(c) every point of $\mathcal{V}$ is connected to some point of $Z$ by an $S^{\sharp}$-path of length at most $C^{\sharp}$ with all points in $\mathcal{V}$.

Put $\delta=\eta$. Then we have $C^{\sharp}$ as above. Let $T \in \overline{\mathcal{F}}(G)$. Then we have $C, n, S_{1}, \ldots, S_{n}$ as given by property $\mathrm{SC}^{\prime}$ for $T_{1}:=\left(S^{\sharp}\right)^{C^{\sharp}} T\left(S^{\sharp}\right)^{C^{\sharp}} \in \overline{\mathcal{F}}(G)$. Put $S_{n+1}=S^{\sharp} \in \overline{\mathcal{F}}(G)$. Now let $G \curvearrowright(X, \mu)$ be an action in $\mathfrak{Y}$. Then we have $Z, \mathcal{V}$ satisfying the above conditions (a)-(c). Put $W_{1}=W_{2}=Z$. Then $\mu\left(W_{1}\right), \mu\left(W_{2}\right) \leq \delta=\eta$. Thus we have $\mathcal{V}_{1}, \ldots, \mathcal{V}_{n}$ satisfying the conditions in Definition 3.12, Put $W=\mathcal{V}$ and $\mathcal{V}_{n+1}=\mathcal{V} \subseteq X$. Then

$$
\sum_{j=1}^{n+1} \Upsilon\left(S_{j}\right) \mu\left(\mathcal{V}_{j}\right) \leq \frac{1}{2}+\Upsilon\left(S^{\sharp}\right) \mu(\mathcal{V}) \leq \frac{1}{2}+\Upsilon\left(S^{\sharp}\right) \varepsilon^{\sharp} \leq \frac{1}{2}+\frac{1}{2}=1,
$$

verifying condition (i) in Definition 3.1. Clearly $S W=S \mathcal{V}=X$, verifying condition (ii) in Definition 3.1. Let $g \in T$ and $w_{1}, w_{2} \in W$ with $g w_{1}=w_{2}$. For $i=1,2$, we can connect $w_{i}$ to a point $z_{i} \in Z$ by an $S_{n+1}$-path of length at most $C^{\sharp}$ with all points in $\mathcal{V}=\mathcal{V}_{n+1}$. Then $z_{i}=s_{i} w_{i}$ for some $s_{i} \in\left(S^{\sharp}\right)^{C^{\sharp}}$. Note that $\left(s_{2} g s_{1}^{-1}\right) z_{1}=z_{2}$ and $s_{2} g s_{1}^{-1} \in\left(S^{\sharp}\right)^{C^{\sharp}} T\left(S^{\sharp}\right)^{C^{\sharp}}=T_{1}$. Since $z_{i} \in W_{i}$, we can connect $z_{1}$ and $z_{2}$ by a path of length at most $C$ in which each edge is an $S_{j}$-edge with both endpoints in $\mathcal{V}_{j}$ for some $1 \leq j \leq n$. Then $w_{1}$ and $w_{2}$ are connected by a path of length at most $C+2 C^{\sharp}$ in which each edge is an $S_{j}$-edge with both endpoints in $\mathcal{V}_{j}$ for some $1 \leq j \leq n+1$, verifying condition (iii) in Definition 3.1. Thus $\mathfrak{Y}$ has property SC.

Now assume that $\mathfrak{Y}$ consists of a single action or of all free p.m.p. actions of $G$. We just need to verify (iii) $\Rightarrow(\mathrm{i})$. The argument in the above paragraph shows that $\mathfrak{Y}$ satisfies the definition of property SC without the bound $C$. From Remark 3.2 we conclude that $\mathfrak{Y}$ has property SC.

\subsection{Normal subgroups and property SC.}

Proposition 3.16. Suppose that $G$ has a normal subgroup $G^{b}$ with property $S C$. Then $G$ has property $S C$.

Proof. By Proposition 3.15 the group $G^{b}$ has the shrinking property. Let $S_{1} \in \overline{\mathcal{F}}\left(G^{b}\right)$ witness the shrinking property for $G^{b}$.

Let $\Upsilon$ be a function $\mathcal{F}(G) \rightarrow[0, \infty)$. Take $0<\varepsilon<1 /\left(3 \Upsilon\left(S_{1}\right)\right)$. Then there is an $S \in \overline{\mathcal{F}}\left(G^{b}\right)$ so that for any $\delta>0$ there is a $C_{1} \in \mathbb{N}$ such that given any free p.m.p. action $G^{b} \curvearrowright(X, \mu)$ we can find Borel sets $Z \subseteq \mathcal{V}_{1} \subseteq X$ satisfying the following conditions:

(i) $S V_{1}=X$,

(ii) $\mu\left(\mathcal{V}_{1}\right) \leq \varepsilon$ and $\mu(Z) \leq \delta$,

(iii) every point of $\mathcal{V}_{1}$ is connected to some point of $Z$ by an $S_{1}$-path of length at most $C_{1}$ with all points in $\mathcal{V}_{1}$. 
Using the function $3 \Upsilon$ in the definition of property SC for $G^{b}$, we find an $S^{b} \in \overline{\mathcal{F}}\left(G^{b}\right)$ such that for any $T^{b} \in \overline{\mathcal{F}}\left(G^{b}\right)$ there are $C^{b}, n^{b} \in \mathbb{N}$, and $S_{1}^{b}, \ldots, S_{n^{b}}^{b} \in \overline{\mathcal{F}}\left(G^{b}\right)$ such that for any free p.m.p. action $G \curvearrowright(X, \mu)$ there are Borel subsets $W^{b}$ and $\mathcal{V}_{k}^{b}$ of $X$ for $1 \leq k \leq n^{b}$ satisfying the following conditions:

(iv) $3 \sum_{k=1}^{n^{b}} \Upsilon\left(S_{k}^{b}\right) \mu\left(\mathcal{V}_{k}^{b}\right) \leq 1$,

(v) $S^{b} W^{b}=X$,

(vi) if $w_{1}, w_{2} \in W^{b}$ satisfy $g w_{1}=w_{2}$ for some $g \in T^{b}$ then $w_{1}$ and $w_{2}$ are connected by a path of length at most $C^{b}$ in which each edge is an $S_{k}^{b}$-edge with both endpoints in $\mathcal{V}_{k}^{b}$ for some $1 \leq k \leq n^{b}$.

Let $T \in \overline{\mathcal{F}}(G)$. Set

$$
S_{2}=S^{b} T \cup\left(S^{b} T\right)^{-1} \in \overline{\mathcal{F}}(G),
$$

and take $0<\delta<1 /\left(3 \Upsilon\left(S_{2}\right)\left|S_{2}\right|\right)$. Then we have $C_{1}$ as above. Set $T^{b}=\bigcup_{g \in T}\left(S^{b} S_{1}^{C_{1}} g S_{1}^{C_{1}} g^{-1} S^{b} \cup\right.$ $\left.S^{b} g S_{1}^{C_{1}} g^{-1} S_{1}^{C_{1}} S^{b}\right) \in \overline{\mathcal{F}}\left(G^{b}\right)$. Then we have $C^{b}, n^{b}$, and $S_{k}^{b}$ for $1 \leq k \leq n^{b}$ as above. Set

$$
C=2 C_{1}+2+C^{b} \in \mathbb{N} .
$$

Now let $G \curvearrowright(X, \mu)$ be a free p.m.p. action. Then we have Borel $Z \subseteq \mathcal{V}_{1} \subseteq X$ satisfying conditions (i)-(iii) above. Note that

$$
\Upsilon\left(S_{1}\right) \mu\left(\mathcal{V}_{1}\right) \leq \Upsilon\left(S_{1}\right) \varepsilon<\frac{1}{3} .
$$

We also have Borel sets $W^{b}$ and $\mathcal{V}_{k}^{b}$ for $1 \leq k \leq n^{b}$ as above, and

$$
\sum_{k=1}^{n^{b}} \Upsilon\left(S_{k}^{b}\right) \mu\left(\mathcal{V}_{k}^{b}\right) \leq \frac{1}{3}
$$

Set $\mathcal{V}_{2}=S_{2} Z$. Then

$$
\Upsilon\left(S_{2}\right) \mu\left(\mathcal{V}_{2}\right) \leq \Upsilon\left(S_{2}\right)\left|S_{2}\right| \mu(Z) \leq \Upsilon\left(S_{2}\right)\left|S_{2}\right| \delta<\frac{1}{3}
$$

Combining all of these bounds we obtain

$$
\Upsilon\left(S_{1}\right) \mu\left(\mathcal{V}_{1}\right)+\Upsilon\left(S_{2}\right) \mu\left(\mathcal{V}_{2}\right)+\sum_{k=1}^{n^{b}} \Upsilon\left(S_{k}^{b}\right) \mu\left(\mathcal{V}_{k}^{b}\right) \leq 1,
$$

which verifies condition (i) in Definition 3.1.

Set $W=\mathcal{V}_{1}$. Then $S W=S \mathcal{V}_{1}=X$, verifying condition (ii) in Definition 3.1.

Let $g \in T$ and $w_{1}, w_{2} \in W$ with $g w_{1}=w_{2}$. For $i=1,2$, we can connect $w_{i}$ to some $z_{i} \in Z$ by an $S_{1}$-path of length at most $C_{1}$ with all points in $\mathcal{V}_{1}$. Then $w_{i}=t_{i} z_{i}$ for some $t_{i} \in S_{1}^{C_{1}}$. Using the fact that $S^{b} W^{b}=X$ we have $g z_{1}=a_{1} u_{1}$ for some $u_{1} \in W^{b}$ and $a_{1} \in S^{b}$, and $z_{2}=a_{2} u_{2}$ for some $u_{2} \in W^{b}$ and $a_{2} \in S^{b}$. Note that $a_{1}^{-1} g$ and $a_{2}^{-1}$ are both in $S_{2}$. Since $\left(a_{1}^{-1} g\right) z_{1}=u_{1}$, the pair $\left(z_{1}, u_{1}\right)$ is an $S_{2}$-edge with both endpoints in $\mathcal{V}_{2}$. Also, since $a_{2}^{-1} z_{2}=u_{2}$, the pair $\left(z_{2}, u_{2}\right)$ is an $S_{2}$-edge with both endpoints in $\mathcal{V}_{2}$. Note that

$$
\left(a_{2}^{-1} t_{2}^{-1} g t_{1} g^{-1} a_{1}\right) u_{1}=a_{2}^{-1} t_{2}^{-1} g t_{1} z_{1}=a_{2}^{-1} t_{2}^{-1} g w_{1}=a_{2}^{-1} t_{2}^{-1} w_{2}=a_{2}^{-1} z_{2}=u_{2} .
$$


Since $a_{2}^{-1} t_{2}^{-1} g t_{1} g^{-1} a_{1} \in S^{b} S_{1}^{C_{1}} g S_{1}^{C_{1}} g^{-1} S^{b} \subseteq T^{b}$, this means that $u_{2} \in T^{b} u_{1}$. Then $u_{1}$ and $u_{2}$ are connected by a path of length at most $C^{b}$ in which each edge is an $S_{k}^{b}$-edge with both endpoints in $\mathcal{V}_{k}^{b}$ for some $1 \leq k \leq n^{b}$. Therefore $w_{1}$ and $w_{2}$ are connected by a path of length at most $2 C_{1}+2+C^{b}=C$ in which each edge is either an $S_{j}$-edge with both endpoints in $\mathcal{V}_{j}$ for some $1 \leq j \leq 2$ or an $S_{k}^{b}$-edge with both endpoints in $\mathcal{V}_{k}^{b}$ for some $1 \leq k \leq n^{b}$, which verifies condition (iii) in Definition 3.1 .

In preparation for the next section we record one application of Proposition 3.16. For this we need the following construction.

Let $G^{b}$ be a finite-index subgroup of $G$. Take a $B \in \mathcal{F}(G)$ such that $G=\bigsqcup_{b \in B} b G^{b}$. Let $G^{b} \curvearrowright(X, \mu)$ be a p.m.p. action. Put $Y=\bigsqcup_{b \in B} b X$ and write $\nu$ for the probability measure on $Y$ which for each $b \in B$ is $\frac{1}{\left[G: G^{b}\right]} \mu$ on $b X$ under the natural identification of $b X$ and $X$. For every $g \in G$ and $b \in B$ we have $g b=b^{\prime} h$ for unique $b^{\prime} \in B$ and $h \in G^{b}$, using which we set

$$
g(b x)=b^{\prime}(h x)
$$

for all $x \in X$. It is easily checked that this defines a p.m.p. action $G \curvearrowright(Y, \nu)$. Furthermore, if $G^{b} \curvearrowright(X, \mu)$ is free then so is $G \curvearrowright(Y, \nu)$.

Proposition 3.17. Let $G^{b}$ be a finite-index subgroup of $G$. Then $G$ has property $S C$ if and only if $G^{b}$ does.

Proof. Assume that $G$ has property SC. Take $B \in \mathcal{F}(G)$ as above containing $e_{G}$. Let $\Upsilon^{b}$ be a function $\mathcal{F}\left(G^{b}\right) \rightarrow[0, \infty)$. For each $F \in \mathcal{F}(G)$, denote by $\varphi(F)$ the smallest element of $\mathcal{F}\left(G^{b}\right)$ satisfying $F \subseteq B \varphi(F)$, and put

$$
\Upsilon(F)=\left[G: G^{b}\right] \Upsilon^{b}\left(\varphi(F B) \cup(\varphi(F B))^{-1}\right) \geq 0 .
$$

Then we have $S \in \overline{\mathcal{F}}(G)$ witnessing property SC for $G$. Put $S^{b}=\varphi(S B) \cup(\varphi(S B))^{-1} \in \overline{\mathcal{F}}\left(G^{b}\right)$. Let $T^{b} \in \overline{\mathcal{F}}\left(G^{b}\right)$. Put $T=B T^{b} B^{-1} \in \overline{\mathcal{F}}(G)$. Then there are $C, n \in \mathbb{N}$ and $S_{1}, \ldots, S_{n} \in \overline{\mathcal{F}}(G)$ satisfying the conditions in Definition 3.1. Put $C^{b}=C, n^{b}=n$, and $S_{j}^{b}=\varphi\left(S_{j} B\right) \cup\left(\varphi\left(S_{j} B\right)\right)^{-1} \in$ $\overline{\mathcal{F}}\left(G^{b}\right)$. Let $G^{b} \curvearrowright(X, \mu)$ be a free p.m.p. action. Define $(Y, \nu)$ as above. Then we have the free action $G \curvearrowright(Y, \nu)$ as above. By property SC there exist Borel subsets $W$ and $\mathcal{V}_{j}$ of $Y$ for $1 \leq j \leq n$ satisfying the following conditions:

(i) $\sum_{j=1}^{n} \Upsilon\left(S_{j}\right) \nu\left(\mathcal{V}_{j}\right) \leq 1$,

(ii) $S W=Y$,

(iii) if $w_{1}, w_{2} \in W$ satisfy $g w_{1}=w_{2}$ for some $g \in T$ then $w_{1}$ and $w_{2}$ are connected by a path of length at most $C$ in which each edge is an $S_{j}$-edge with both endpoints in $V_{j}$ for some $1 \leq j \leq n$.

We can write $W$ as $\bigsqcup_{b \in B} b W_{b}$ for some Borel sets $W_{b} \subseteq X$. Put $W^{b}=\bigcup_{b \in B} W_{b} \subseteq X$. Similarly, for each $1 \leq j \leq n$ we can write $\mathcal{V}_{j}$ as $\bigsqcup_{b \in B} b \mathcal{V}_{j, b}$ for some Borel sets $\mathcal{V}_{j, b} \subseteq X$, and we put $\mathcal{V}_{j}^{b}=\bigcup_{b \in B} \mathcal{V}_{j, b} \subseteq X$. We then have

$$
\sum_{j=1}^{n^{\mathrm{b}}} \Upsilon^{\mathrm{b}}\left(S_{j}^{\mathrm{b}}\right) \mu\left(\mathcal{V}_{j}^{\mathrm{b}}\right)=\frac{1}{\left[G: G^{\mathrm{b}}\right]} \sum_{j=1}^{n} \Upsilon\left(S_{j}\right) \mu\left(\mathcal{V}_{j}^{\mathrm{b}}\right) \leq \sum_{j=1}^{n} \Upsilon\left(S_{j}\right) \nu\left(\mathcal{V}_{j}\right) \leq 1,
$$


verifying condition (i) in Definition 3.1. Clearly $S^{b} W^{b}=X$, verifying condition (ii) in Definition 3.1 .

Let $g \in T^{b}$ and $w_{1}, w_{2} \in W^{b}$ with $g w_{1}=w_{2}$. Then there are $b_{1}, b_{2} \in B$ such that $b_{1} w_{1}, b_{2} w_{2} \in$ $W$. Note that $\left(b_{2} g b_{1}^{-1}\right) b_{1} w_{1}=b_{2} w_{2}$, and $b_{2} g b_{1}^{-1} \in T$. Then there are $1 \leq l \leq C$ and $1 \leq$ $j_{1}, \ldots, j_{l} \leq n$, and $g_{i} \in S_{j_{i}}$ for $1 \leq i \leq l$ such that $g_{i-1} \ldots g_{1} b_{1} w_{1}, g_{i} \ldots g_{1} b_{1} w_{1} \in \mathcal{V}_{j_{i}}$ for all $1 \leq i \leq l$, and $g_{l} \ldots g_{1} b_{1} w_{1}=b_{2} w_{2}$. Recursively define $h_{i} \in G^{b}$ and $d_{i} \in B$ for $i=1, \ldots, l$ by $g_{i} d_{i-1}=d_{i} h_{i}$ and $d_{0}=b_{1}$. Then $h_{i} \in S_{j_{i}}^{b}$ and $g_{i} \ldots g_{1} b_{1} w_{1}=d_{i} h_{i} \ldots h_{1} w_{1}$ for all $1 \leq i \leq l$. Thus $h_{i-1} \ldots h_{1} w_{1}, h_{i} \ldots h_{1} w_{1} \in \mathcal{V}_{j_{i}}^{b}$ for all $1 \leq j \leq l$. Also, from $b_{2} w_{2}=g_{l} \ldots g_{1} b_{1} w_{1}=d_{l} h_{l} \ldots h_{1} w_{1}$ we get $w_{2}=h_{l} \ldots h_{1} w_{1}$. Thus $w_{1}$ and $w_{2}$ are connected by a path of length at most $C^{b}$ in which each edge is an $S_{j}^{b}$-edge with both endpoints in $\mathcal{V}_{j}^{b}$ for some $1 \leq j \leq n^{b}$, verifying condition (iii) in Definition 3.1. Therefore $G^{b}$ has property SC. This proves the "only if" part.

Suppose now that $G^{b}$ has property SC. Since $G^{b}$ has finite index in $G$, we can find a finiteindex normal subgroup $G^{\prime}$ of $G$ such that $G^{\prime} \subseteq G^{b}$. By the "only if" part, $G^{\prime}$ has property SC. By Proposition 3.16, the group $G$ has property SC. This establishes the "if" part.

\subsection{Groups without property SC.}

Lemma 3.18. Let $\Gamma$ be a (not necessarily infinite) countable group. Let $\mathfrak{Y}_{G * \Gamma}$ be a class of free p.m.p. actions of $G * \Gamma$. Denote by $\mathfrak{Y}_{G}$ the class of restriction actions $G \curvearrowright(X, \mu)$ for

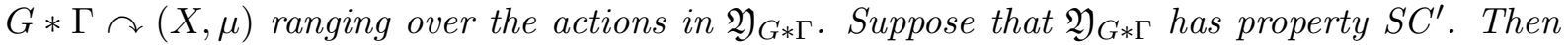
$\mathfrak{Y}_{G}$ has property $S C^{\prime}$.

Proof. We may assume that $\Gamma$ is nontrivial. For any $A \in \overline{\mathcal{F}}(G), B \in \overline{\mathcal{F}}(\Gamma)$, and $k \in \mathbb{N}$ we denote by $A *_{k} B$ the subset of $G * \Gamma$ consisting of all elements of the form $a_{k} b_{k} a_{k-1} b_{k-1} \ldots a_{1} b_{1}$ for $a_{1}, \ldots, a_{k} \in A$ and $b_{1}, \ldots, b_{k} \in B$. For each $F \in \mathcal{F}(G * \Gamma)$ we take some $k \in \mathbb{N}, A \in \overline{\mathcal{F}}(G)$, and $B \in \overline{\mathcal{F}}(\Gamma)$ such that $F \subseteq A *_{k} B$, and we put $\Phi(F)=A *_{k} B \in \mathcal{F}(G * \Gamma)$.

Let $\Upsilon_{G}: \mathcal{F}(G) \rightarrow[0, \infty)$ be a function. Define $\Upsilon_{G * \Gamma}: \mathcal{F}(G * \Gamma) \rightarrow[0, \infty)$ by $\Upsilon_{G * \Gamma}(F)=$ $|\Phi(F)| \Upsilon_{G}(A)$, where $\Phi(F)=A *_{k} B$.

Let $\eta_{G * \Gamma}>0$ witness property $\mathrm{SC}^{\prime}$ for $\mathfrak{Y}_{G * \Gamma}$. Put $\eta_{G}=\eta_{G * \Gamma}>0$.

Let $T_{G} \in \overline{\mathcal{F}}(G)$. Put $T_{G * \Gamma}=T_{G} \in \overline{\mathcal{F}}(G * \Gamma)$. Then we have $n, C_{G * \Gamma} \in \mathbb{N}$, and $S_{G * \Gamma, 1}, \ldots, S_{G * \Gamma, n} \in$ $\overline{\mathcal{F}}(G * \Gamma)$ witnessing property $\mathrm{SC}^{\prime}$ for $\mathfrak{Y}_{G * \Gamma}$. For $1 \leq j \leq n$ express $\Phi\left(S_{G * \Gamma, j}\right)$ as $A_{j} *_{k_{j}} B_{j}$ as per its definition. Put $S_{G, j}=A_{j} \in \overline{\mathcal{F}}(G)$ for $1 \leq j \leq n$. Also, put $C_{G}=2 C_{G * \Gamma} \max _{1 \leq j \leq n} k_{j} \in \mathbb{N}$.

Let $G * \Gamma \curvearrowright(X, \mu)$ be an action in $\mathfrak{Y}_{G * \Gamma}$. Let $W_{1}$ and $W_{2}$ be Borel subsets of $X$ with $\mu\left(W_{1}\right), \mu\left(W_{2}\right) \leq \eta_{G}=\eta_{G * \Gamma}$. Then there are Borel sets $\mathcal{V}_{G * \Gamma, 1}, \ldots, \mathcal{V}_{G * \Gamma, n} \subseteq X$ satisfying the following conditions:

(i) $\sum_{j=1}^{n} \mu\left(\mathcal{V}_{G * \Gamma, j}\right) \Upsilon_{G * \Gamma}\left(S_{G * \Gamma, j}\right) \leq 1$,

(ii) if $w_{1} \in W_{1}$ and $w_{2} \in W_{2}$ satisfy $g w_{1}=w_{2}$ for some $g \in T_{G * \Gamma}$ then $w_{1}$ and $w_{2}$ are connected by a path of length at most $C_{G * \Gamma}$ in which each edge is an $S_{G * \Gamma, j}$-edge with both endpoints in $\mathcal{V}_{G * \Gamma, j}$ for some $1 \leq j \leq n$.

For each $1 \leq j \leq n$ put $\mathcal{V}_{G, j}=\Phi\left(S_{G * \Gamma, j}\right) \mathcal{V}_{G * \Gamma, j} \subseteq X$. Then

$$
\sum_{j=1}^{n} \mu\left(\mathcal{V}_{G, j}\right) \Upsilon_{G}\left(S_{G, j}\right) \leq \sum_{j=1}^{n} \mu\left(\mathcal{V}_{G * \Gamma, j}\right)\left|\Phi\left(S_{G * \Gamma, j}\right)\right| \Upsilon_{G}\left(S_{G, j}\right)
$$




$$
=\sum_{j=1}^{n} \mu\left(\mathcal{V}_{G * \Gamma, j}\right) \Upsilon_{G * \Gamma}\left(S_{G * \Gamma, j}\right) \leq 1,
$$

verifying condition (i) in Definition 3.12 ,

For each $1 \leq j \leq n$, if $h \in S_{G * \Gamma, j}$ and $x, y \in \mathcal{V}_{G * \Gamma, j}$ with $h x=y$ then clearly $x$ and $y$ are connected by an $\left(A_{j} \cup B_{j}\right)$-path in $\mathcal{V}_{G, j}$ of length at most $2 k_{j}$.

Let $g \in T_{G}=T_{G * \Gamma}$ and $w_{1} \in W_{1}, w_{2} \in W_{2}$ with $g w_{1}=w_{2}$. Then $w_{1}$ and $w_{2}$ are connected by a path of length at most $C_{G * \Gamma}$ in which each edge is an $S_{G * \Gamma, j}$-edge with both endpoints in $\mathcal{V}_{G * \Gamma, j}$ for some $1 \leq j \leq n$. From the above paragraph we conclude that $w_{1}$ and $w_{2}$ are connected by a path of length at most $C_{G}$ in which each edge is either an $A_{j}$-edge or a $B_{j}$-edge with both endpoints in $\mathcal{V}_{G, j}$ for some $1 \leq j \leq n$. We may assume that $w_{1} \neq w_{2}$. Removing cycles in this path, we may assume that it contains no cycles. Since $g \in G$ and the action $G * \Gamma \curvearrowright(X, \mu)$ is free, we see that no $B_{j}$-edge for any $1 \leq j \leq n$ appears in this path. Thus each edge of this path is an $S_{G, j}$-edge with both endpoints in $\mathcal{V}_{G, j}$ for some $1 \leq j \leq n$. This verifies condition (ii) in Definition 3.12.

By combining Proposition 3.15 and Lemmas 3.13, 3.14, and 3.18 we obtain the following proposition, which, with a boost from Proposition 3.17, then yields Theorem 3.20 .

Proposition 3.19. Suppose that $G$ is either locally finite or virtually cyclic, and let $\Gamma$ be a (not necessarily infinite) countable group. Then no free p.m.p. action $G * \Gamma \curvearrowright(X, \mu)$ has property $S C$.

Theorem 3.20. Suppose that $G$ is either locally finite or virtually free. Then $G$ does not have property $S C$.

We will see later in Proposition 3.28 that if $G$ is amenable then it has property SC if and only if it is neither virtually cyclic nor locally finite.

3.6. Groups with property SC. The following notion of w-normality was formulated by Popa for the purpose of expressing his cocycle superrigidity theorem in [31] and will be similarly convenient in our setting.

Definition 3.21. A subgroup $G_{0}$ of $G$ is w-normal in $G$ if there are a countable ordinal $\gamma$ and a subgroup $G_{\lambda}$ of $G$ for each ordinal $0 \leq \lambda \leq \gamma$ satisfying the following conditions:

(i) for any $\lambda<\lambda^{\prime} \leq \gamma$ one has $G_{\lambda} \subseteq G_{\lambda^{\prime}}$,

(ii) $G=G_{\gamma}$,

(iii) for each $\lambda<\gamma, G_{\lambda}$ is normal in $G_{\lambda+1}$,

(iv) for each limit ordinal $\lambda^{\prime} \leq \gamma, G_{\lambda^{\prime}}=\bigcup_{\lambda<\lambda^{\prime}} G_{\lambda}$.

Our goal is to prove, via several lemmas culminating in Theorem [3.29, that if $G$ contains an amenable w-normal subgroup which is neither locally finite nor virtually cyclic then $G$ has property SC. This will involve an elaboration of the graph-theoretic arguments from Section 8.1 of 2 .

Let $S$ be a symmetric finite subset of $G$. By an $S$-path in $G$ we mean a finite tuple $\left(g_{0}, g_{1}, \ldots, g_{n}\right)$ of elements of $G$ such that $g_{i-1} g_{i}^{-1} \in S$ for all $i=1, \ldots, n$, in which case we call $n$ the length of the path and say that the path connects $g_{0}$ and $g_{n}$ (its endpoints). We say that a set $K \subseteq G$ is $S$-connected if every pair of distinct elements of $K$ is connected by an $S$-path. 
Given an $r \in \mathbb{N}$, a set $E \subseteq G$ is said to be $(S, r)$-separated if for all distinct $f, g \in E$ one has $S^{r} f \cap S^{r} g=\emptyset$. Given a set $W \subseteq G$ and an $r \in \mathbb{N}$, a set $E \subseteq W$ is said to be $(S, r)$-spanning for $W$ if every $f \in W$ is connected to some $g \in E$ by an $S$-path of length at most $r$. We also simply say $S$-spanning when $r=1$. This should not be confused with the graph-theoretic notion of spanning tree, which we also use below.

For finite sets $F, K \subseteq G$ and $\delta>0$, we say that $K$ is $(F, \delta)$-invariant if $|F K \backslash K|<\delta|K|$. Note that, given an $r \in \mathbb{N}$, if $F$ contains $e_{G}$ and satisfies $|F|>1$ and $K$ is $\left(F, \delta^{\prime}\right)$-invariant where $\delta^{\prime}=\delta(1-|F|) /\left(1-|F|^{r}\right)$ then $K$ is $\left(F^{r}, \delta\right)$-invariant, for using the fact that $F$ contains $e_{G}$ we have

$$
\left|F^{r} K \backslash K\right|=\sum_{j=1}^{r}\left|F^{j} K \backslash F^{j-1} K\right| \leq \sum_{j=1}^{r}\left|F^{j-1}\right| F K \backslash K|<\delta| K \mid .
$$

The following is a variation on Lemma 8.3 of [2]. For an $\varepsilon \geq 0$ and a collection $\mathscr{T}$ of finite subsets of $G$, we say a finite set $K \subseteq G$ is tiled to within $\varepsilon$ by $\mathscr{T}$ if the members of $\mathscr{T}$ are pairwise disjoint subsets of $K$ and $\left|\bigsqcup_{T \in \mathscr{T}} T\right| \geq(1-\varepsilon)|K|$.

Lemma 3.22. Suppose that $G$ is finitely generated and let $S$ be a finite symmetric generating set for $G$ containing $e_{G}$. Let $\varepsilon>0$. Let $F$ be a finite subset of $G$ and $\delta>0$. Then there exists $a \zeta>0$ such that every $(S, \zeta)$-invariant nonempty finite subset of $G$ is tiled to within $\varepsilon$ by a collection of $S^{2}$-connected $(F, \delta)$-invariant finite subsets of $G$.

Proof. Take an $r \in \mathbb{N}$ such that $F \subseteq S^{r}$. Set $\zeta=\min \left\{\varepsilon^{2},\left(\delta(1-|S|) /\left(1-|S|^{r}\right)\right)^{2}\right\}$. Let $K$ be an $(S, \zeta)$-invariant nonempty finite subset of $G$. Consider the partition $K_{1} \sqcup \cdots \sqcup K_{n}$ of $K$ into maximal $S^{2}$-connected subsets. Then $S K_{i} \cap S K_{j}=\emptyset$ for $i \neq j$, and so

$$
\sum_{i=1}^{n} \frac{\left|K_{i}\right|}{|K|} \cdot \frac{\left|S K_{i} \backslash K_{i}\right|}{\left|K_{i}\right|}=\frac{|S K \backslash K|}{|K|}<\zeta .
$$

Write $I$ for the set of all $i \in\{1, \ldots, n\}$ such that $\left|S K_{i} \backslash K_{i}\right| /\left|K_{i}\right|<\sqrt{\zeta}$. Then by (2) we must have $\sum_{i \in I}\left|K_{i}\right| \geq(1-\sqrt{\zeta})|K|$, which shows, since $\sqrt{\zeta} \leq \varepsilon$, that the collection $\left\{K_{i}\right\}_{i \in I}$ tiles $K$ to within $\varepsilon$. Moreover, since $\sqrt{\zeta} \leq \delta(1-|S|) /\left(1-|S|^{r}\right)$ and $F \subseteq S^{r}$ we infer from the observation before the lemma that for each $i \in I$ the set $K_{i}$ is $(F, \delta)$-invariant, completing the proof.

We next state a version of the Ornstein-Weiss tiling theorem that demands connectedness of the tiles. It follows from one of the usual forms of the Ornstein-Weiss tiling theorem (Theorem 4.46 of [24]) and Lemma 3.22.

Lemma 3.23. Suppose that $G$ is amenable and finitely generated. Let $S$ be a finite symmetric generating set for $G$ containing $e_{G}$. Let $E$ be a finite subset of $G$ and $\delta>0$. Then there are $(E, \delta)$-invariant $S^{2}$-connected sets $F_{1}, \ldots, F_{m} \in \mathcal{F}(G)$ such that for any free p.m.p. action $G \curvearrowright(X, \mu)$ we can find Borel sets $Z_{1}, \ldots, Z_{m} \subseteq X$ such that the collection $\left\{F_{k} z: 1 \leq k \leq\right.$ $m$ and $\left.z \in Z_{k}\right\}$ is disjoint and its union has $\mu$-measure at least $1-\delta$.

The following is essentially Lemma 8.5 of [2].

Lemma 3.24. Suppose that $G$ is finitely generated and not virtually cyclic, and let $A$ be a generating set for $G$ in $\overline{\mathcal{F}}(G)$. Then there is a constant $b>0$ such that given any $r \in \mathbb{N}$ and $A$-connected finite set $F \subseteq G$ satisfying $\left|A^{r} F\right| \leq 2|F|$ there exists an A-connected set $T \subseteq A^{r} F$ such that $T \cap F$ is $(A, 2 r)$-spanning for $F$ and $|T| \leq b|F| / r$. 
Proof. As $G$ is not virtually cyclic, we can find a $c>0$ such that $\left|A^{n}\right| \geq c n^{2}$ for all $n \in \mathbb{N}$ (Corollary 3.5 of [27]). Let $r$ and $F$ be as in the lemma statement.

Take a maximal $(A, r)$-separated subset $V$ of $F$. Then we have

$$
|V|\left|A^{r}\right|=\left|\bigsqcup_{g \in V} A^{r} g\right| \leq\left|A^{r} F\right| \leq 2|F|
$$

whence

$$
|V| \leq \frac{2|F|}{\left|A^{r}\right|} \leq \frac{2}{c r^{2}}|F|
$$

The set $V$ is $(A, 2 r)$-spanning for $F$ by maximality. Consider the graph $(V, E)$ whose edges are those pairs of vertices which can be joined by an $A$-path within $A^{r} F$ of length at most $4 r+1$. Since $F$ is $A$-connected, for all $v_{1}, v_{2} \in V$ there is an $A$-path in $F$ connecting $v_{1}$ to $v_{2}$. For each point $z$ in this path, we may connect $z$ to some $v_{z} \in V$ by an $A$-path $p_{z}$ in $A^{r} F$ of length at most $2 r$ (using the $(A, 2 r)$-spanningness of $V$ in $F$ and the fact that an $A$-path of length at most $2 r$ with both endpoints in $F$ must be entirely contained in $A^{r} F$ ) we can construct an $A$-path in $A^{r} F$ from $v_{1}$ to $v_{2}$ in which points of $V$ appear in every interval of length $4 r+1$, by inserting $p_{z}$ and the reverse of $p_{z}$ at $z$. This shows that the graph $(V, E)$ is connected.

Applying the standard procedure for producing a spanning tree, we start with $(V, E)$ and then recursively construct a sequence of graphs with vertex set $V$ by removing one edge at each stage so as to destroy some cycle in the graph at that stage, until there are no more cycles to destroy and we arrive at a spanning tree $\left(V, E^{\prime}\right)$. Then $\left(V, E^{\prime}\right)$ is an $A^{4 r+1}$-tree in $F$ which is $(A, 2 r)$-spanning for $F$.

For each pair $(v, w)$ in $E^{\prime}$, choose an $A$-path in $A^{r} F$ joining $v$ to $w$ of length at most $4 r+1$. Denote by $T$ the collection of all vertices which appear in one of these paths. Note that $T$ is an $A$-connected set in $A^{r} F$ such that $T \cap F$ is $(A, 2 r)$-spanning for $F$. Moreover, using (3) we have

$$
|T| \leq|V|+4 r\left|E^{\prime}\right| \leq(4 r+1)|V| \leq 5 r \cdot \frac{2}{c r^{2}}|F|=\frac{10}{c r}|F| .
$$

We can therefore take $b=10 / c$.

If we take $G^{b}=G$ in Proposition 3.16, then the proof there actually shows the following.

Lemma 3.25. Let $G_{0}$ be a subgroup of $G$ which has the shrinking property. Then in the definition of property $S C$ for a class $\mathfrak{Y}$ of free p.m.p. actions of $G$ (Definition 3.1) it is possible, for each $\Upsilon$, to choose $S$ to be a subset of $G_{0}$ depending only on $\left.\Upsilon\right|_{\mathcal{F}\left(G_{0}\right)}$.

Lemma 3.26. Let $\gamma$ be a countable ordinal, and suppose that for each ordinal $\lambda<\gamma$ there is a subgroup $G_{\lambda}$ of $G$ so that the following conditions hold:

(i) for any $\lambda<\lambda^{\prime}<\alpha$ one has $G_{\lambda} \subseteq G_{\lambda^{\prime}}$,

(ii) $G_{\lambda}$ has property $S C$ for every $\lambda<\gamma$.

Then $\bigcup_{\lambda<\gamma} G_{\lambda}$ has property $S C$.

Proof. By Proposition 3.15 we know that $G_{0}$ has the shrinking property. Let $\Upsilon$ be a function $\mathcal{F}\left(\bigcup_{\lambda<\gamma} G_{\lambda}\right) \rightarrow[0, \infty)$. By Lemma 3.25 there is some $S \in \overline{\mathcal{F}}\left(G_{0}\right)$ witnessing property SC for $G_{\lambda}$ with respect to $\left.\Upsilon\right|_{\mathcal{F}\left(G_{\lambda}\right)}$ for all $\lambda<\gamma$. Let $T \in \overline{\mathcal{F}}\left(\bigcup_{\lambda<\gamma} G_{\lambda}\right)$. Then $T \in \overline{\mathcal{F}}\left(G_{\lambda}\right)$ for some $\lambda<\gamma$. Then we have $C, n \in \mathbb{N}$ and $S_{1}, \ldots, S_{n} \in \overline{\mathcal{F}}\left(G_{\lambda}\right)$ satisfying the conditions in Definition 3.1. 
Lemma 3.27. Suppose that $G$ is amenable and not locally virtually cyclic. Then $G$ has property $S C$.

Proof. We consider first the case $G$ is finitely generated. Then $G$ is not virtually cyclic. By Proposition 3.15 it suffices to show that $G$ has property $\mathrm{SC}^{\prime}$.

Take an $S_{0} \in \overline{\mathcal{F}}(G)$ generating $G$. Set $S_{1}=S_{0}^{2} \in \overline{\mathcal{F}}(G)$. Let $b>0$ be as given by Lemma 3.24 with respect to the generating set $S_{1}$ for $G$.

Let $\Upsilon$ be a function $\mathcal{F}(G) \rightarrow[0, \infty)$. Choose an $r \in \mathbb{N}$ large enough so that

$$
3 b \Upsilon\left(S_{1}\right) \leq r
$$

Set $S_{2}=S_{1}^{2 r} \in \overline{\mathcal{F}}(G)$. Take $\eta>0$ such that $2 \eta\left|S_{2}\right| \Upsilon\left(S_{2}\right)<1 / 3$.

Let $T \in \overline{\mathcal{F}}(G)$. Put $n=3$ and $S_{3}=T \in \overline{\mathcal{F}}(G)$. Take $0<\delta<1$ such that $2 \delta\left|S_{3}\right| \Upsilon\left(S_{3}\right)<1 / 3$ and put $\zeta=\delta /\left(1+\left|S_{2}\right| \cdot\left|S_{2} T S_{2}\right|\right)>0$. By Lemma 3.23 there are $m \in \mathbb{N}$ and $\left(S_{2} T S_{2}, \zeta\right)$-invariant $S_{1}$-connected sets $F_{1}, \ldots, F_{m} \in \mathcal{F}(G)$ such that for any free p.m.p. action $G \curvearrowright(X, \mu)$ we can find Borel sets $Z_{1}, \ldots, Z_{m} \subseteq X$ such that the collection $\left\{F_{k} z: 1 \leq k \leq m\right.$ and $\left.z \in Z_{k}\right\}$ is disjoint and its union has $\mu$-measure at least $1-\zeta$. By our choice of $b$ via Lemma 3.24 , for each $k=1, \ldots, m$ we can find an $S_{1}$-connected set $T_{k}^{*} \subseteq S_{1}^{r} F_{k}$ such that $T_{k}^{\dagger}:=T_{k}^{*} \cap F_{k}$ is $\left(S_{1}, 2 r\right)$-spanning for $F_{k}$ and $\left|T_{k}^{*}\right| \leq b\left|F_{k}\right| / r$. Put $C=2+\max _{1 \leq k \leq m}\left|T_{k}^{*}\right| \in \mathbb{N}$. For each $1 \leq k \leq m$ denote by $F_{k}^{\prime}$ the set of all $g \in F_{k}$ satisfying $S_{2} T S_{2} g \subseteq F_{k}$, and note that since $F_{k}$ is $\left(S_{2} T S_{2}, \zeta\right)$-invariant we have $\left|F_{k} \backslash F_{k}^{\prime}\right| \leq\left|S_{2} T S_{2}\right| \zeta\left|F_{k}\right|$.

Now let $G \curvearrowright(X, \mu)$ be a free p.m.p. action, and let $W_{1}, W_{2}$ be Borel subsets of $X$ with $\mu\left(W_{1}\right), \mu\left(W_{2}\right) \leq \eta$. Take $Z_{1}, \ldots, Z_{m}$ as above.

For each $i=1,2$ put $W_{i}^{\prime}=W_{i} \cap S_{2} \bigcup_{k=1}^{m}\left(F_{k}^{\prime} \cap T_{k}^{\dagger}\right) Z_{k} \subseteq W_{i}$ and observe that

$$
\begin{aligned}
\mu\left(W_{i} \backslash W_{i}^{\prime}\right) & \leq \mu\left(X \backslash S_{2} \bigcup_{k=1}^{m}\left(F_{k}^{\prime} \cap T_{k}^{\dagger}\right) Z_{k}\right) \\
& \leq \mu\left(X \backslash \bigcup_{k=1}^{m} F_{k} Z_{k}\right)+\sum_{k=1}^{m} \mu\left(F_{k} Z_{k} \backslash S_{2}\left(F_{k}^{\prime} \cap T_{k}^{\dagger}\right) Z_{k}\right) \\
& \leq \zeta+\sum_{k=1}^{m} \mu\left(Z_{k}\right)\left|F_{k} \backslash S_{2}\left(F_{k}^{\prime} \cap T_{k}^{\dagger}\right)\right| \\
& \leq \zeta+\sum_{k=1}^{m} \mu\left(Z_{k}\right)\left|S_{2}\right| \cdot\left|T_{k}^{\dagger} \backslash F_{k}^{\prime}\right| \quad\left(\text { since } F_{k} \subseteq S_{2} T_{k}^{\dagger}\right) \\
& \leq \zeta+\left|S_{2} T S_{2}\right| \zeta \sum_{k=1}^{m} \mu\left(Z_{k}\right)\left|S_{2}\right| \cdot\left|F_{k}\right| \\
& \leq \zeta+\left|S_{2} T S_{2}\right| \cdot\left|S_{2}\right| \zeta=\delta .
\end{aligned}
$$

Set

$$
\mathcal{V}_{1}=\bigcup_{k=1}^{m} T_{k}^{*} Z_{k}, \quad \mathcal{V}_{2}=S_{2}\left(W_{1} \cup W_{2}\right), \quad \mathcal{V}_{3}=S_{3}\left(\left(W_{1} \backslash W_{1}^{\prime}\right) \cup\left(W_{2} \backslash W_{2}^{\prime}\right)\right)
$$


Then

$$
\Upsilon\left(S_{1}\right) \mu\left(\mathcal{V}_{1}\right) \leq \Upsilon\left(S_{1}\right) \sum_{k=1}^{m} \mu\left(Z_{k}\right)\left|T_{k}^{*}\right| \leq \Upsilon\left(S_{1}\right) \sum_{k=1}^{m} \mu\left(Z_{k}\right) \frac{b\left|F_{k}\right|}{r} \leq \Upsilon\left(S_{1}\right) \frac{b}{r} \stackrel{\text { (4) }}{\leq} \frac{1}{3}
$$

and

$$
\Upsilon\left(S_{2}\right) \mu\left(\mathcal{V}_{2}\right) \leq \Upsilon\left(S_{2}\right)\left|S_{2}\right|\left(\mu\left(W_{1}\right)+\mu\left(W_{2}\right)\right) \leq 2 \eta\left|S_{2}\right| \Upsilon\left(S_{2}\right) \leq \frac{1}{3}
$$

and

$$
\Upsilon\left(S_{3}\right) \mu\left(\mathcal{V}_{3}\right) \leq \Upsilon\left(S_{3}\right)\left|S_{3}\right|\left(\mu\left(W_{1} \backslash W_{1}^{\prime}\right)+\mu\left(W_{2} \backslash W_{2}^{\prime}\right)\right) \leq 2 \delta\left|S_{3}\right| \Upsilon\left(S_{3}\right)<\frac{1}{3}
$$

so that

$$
\sum_{j=1}^{3} \Upsilon\left(S_{j}\right) \mu\left(\mathcal{V}_{j}\right) \leq 1
$$

which verifies condition (i) in Definition 3.12 .

Let $g \in T$ and $w_{1} \in W_{1}, w_{2} \in W_{2}$ with $g w_{1}=w_{2}$. If $w_{1} \notin W_{1}^{\prime}$ or $w_{2} \notin W_{2}^{\prime}$, then $\left(w_{1}, w_{2}\right)$ is an $S_{3}$-edge with both endpoints in $\mathcal{V}_{3}$. Thus we may assume that $w_{i} \in W_{i}^{\prime}$ for $i=1,2$. For $i=1,2$, we have $w_{i}=s_{i} t_{i} z_{i}$ for some $s_{i} \in S_{2}, 1 \leq k_{i} \leq m, t_{i} \in F_{k_{i}}^{\prime} \cap T_{k_{i}}^{\dagger}$, and $z_{i} \in Z_{k_{i}}$. Then $\left(w_{i}, t_{i} z_{i}\right)$ is an $S_{2}$-edge with both endpoints in $\mathcal{V}_{2}$. Note that $s_{2}^{-1} g s_{1} \in S_{2} T S_{2}$, and hence $\left(s_{2}^{-1} g s_{1}\right) t_{1} \in F_{k_{1}}$. Since

$$
t_{2} z_{2}=s_{2}^{-1} w_{2}=s_{2}^{-1} g w_{1}=\left(s_{2}^{-1} g s_{1}\right) t_{1} z_{1},
$$

we get that $k_{1}=k_{2}, t_{2}=\left(s_{2}^{-1} g s_{1}\right) t_{1}$, and $z_{2}=z_{1}$. Then $t_{1} z_{1}$ and $t_{2} z_{2}$ are connected by an $S_{1}$-path of length at most $\left|T_{k_{1}}^{*}\right|$ with all points in $\mathcal{V}_{1}$. Therefore $w_{1}$ and $w_{2}$ are connected by a path of length at most $C$ in which each edge is an $S_{j}$-edge with both endpoints in $\mathcal{V}_{j}$ for some $1 \leq j \leq 3$, verifying condition (ii) in Definition 3.12 , Therefore $G$ has property $\mathrm{SC}^{\prime}$, and hence has property SC.

Now consider the case $G$ is not finitely generated. By hypothesis we can find an increasing sequence $G_{0} \subseteq G_{1} \subseteq \ldots$ of finitely generated subgroups of $G$ with union $G$ such that $G_{0}$ is not virtually cyclic. Suppose for a given $n \in \mathbb{N}$ that $G_{n}$ is virtually cyclic. Then $G_{n}$ has a finite-index subgroup $G_{n}^{\prime}$ isomorphic to $\mathbb{Z}$. Since $G_{0}$ is infinite, $G_{0} \cap G_{n}^{\prime}$ is nontrivial. Then $G_{0} \cap G_{n}^{\prime}$ has finite index in $G_{n}^{\prime}$, and hence has finite index in $G_{n}$. Thus $G_{0} \cap G_{n}^{\prime}$ has finite index in $G_{0}$. This shows that $G_{0}$ is virtually cyclic, a contradiction. Therefore for each $n \in \mathbb{N}$ the group $G_{n}$ is not virtually cyclic. Since subgroups of amenable groups are amenable, each $G_{n}$ is amenable. From the finitely generated case of the lemma we conclude that each $G_{n}$ has property SC. Then from Lemma 3.26 we get that $G$ has property SC.

The following we obtain from Theorem 3.20 and Lemmas 3.8 and 3.27

Proposition 3.28. An amenable $G$ has property $S C$ if and only if it is neither locally finite nor virtually cyclic.

From Proposition 3.28 and Lemma 3.26 we finally get:

Theorem 3.29. Suppose that $G$ has a w-normal subgroup $G_{0}$ which is amenable but neither locally finite nor virtually cyclic. Then $G$ has property $S C$. 


\subsection{Product groups.}

Lemma 3.30. For every $\varepsilon>0$ there is an $S \in \overline{\mathcal{F}}(G)$ such that for every free p.m.p. action $G \curvearrowright(X, \mu)$ there is a Borel set $\mathcal{V} \subseteq X$ with $S \mathcal{V}=X$ and $\mu(\mathcal{V}) \leq \varepsilon$.

Proof. By Proposition 3.11, $G$ either has the shrinking property or is amenable. If $G$ has the shrinking property the lemma is clear, while if $G$ is amenable then the lemma follows from the Ornstein-Weiss quasitower theorem [24, Theorem 4.46].

For two countably infinite groups $G$ and $\Gamma$, we say that $G \times \Gamma$ has property $S C$ for product actions if the class $\mathfrak{Y}_{\text {prod }}$ has property SC, where $\mathfrak{Y}_{\text {prod }}$ consists of all p.m.p. actions of the form $G \times \Gamma \curvearrowright(X \times Y, \mu)$ where $G \curvearrowright X$ and $G \curvearrowright Y$ are free actions on standard Borel spaces and $G \times \Gamma \curvearrowright X \times Y$ is the product action, with $\mu$ not necessarily being a product measure.

Lemma 3.31. Let $G$ and $\Gamma$ be countably infinite groups. Suppose that $G$ has the shrinking property. Then $G \times \Gamma$ has property $S C$ for product actions.

Proof. We have $S_{1, G} \in \overline{\mathcal{F}}(G)$ witnessing the shrinking property for $G$. Put $S_{1}=S_{1, G} \times\left\{e_{\Gamma}\right\} \in$ $\overline{\mathcal{F}}(G \times \Gamma)$. Let $\Upsilon$ be a function $\mathcal{F}(G \times \Gamma) \rightarrow[0, \infty)$. Take $\varepsilon>0$ such that $\varepsilon \Upsilon\left(S_{1}\right) \leq 1 / 3$.

By our choice of $S_{1, G}$, there is an $S_{G} \in \overline{\mathcal{F}}(G)$ such that for any $\delta>0$ there is a $C_{1} \in \mathbb{N}$ so that for any free p.m.p. action $G \curvearrowright\left(X, \mu_{X}\right)$ we can find Borel sets $Z_{X} \subseteq \mathcal{V}_{X} \subseteq X$ satisfying the following conditions:

(i) $S_{G} \mathcal{V}_{X}=X$

(ii) $\mu_{X}\left(\mathcal{V}_{X}\right) \leq \varepsilon$ and $\mu_{X}\left(Z_{X}\right) \leq \delta$,

(iii) every point of $\mathcal{V}_{X}$ is connected to some point of $Z_{X}$ by an $S_{1, G^{-}}$path of length at most $C_{1}$ with all points in $\mathcal{V}_{X}$.

Put $S=S_{G} \times\left\{e_{\Gamma}\right\} \in \overline{\mathcal{F}}(G \times \Gamma)$.

Let $T \in \overline{\mathcal{F}}(G \times \Gamma)$. Take $T_{G} \in \overline{\mathcal{F}}(G)$ and $T_{\Gamma} \in \overline{\mathcal{F}}(\Gamma)$ such that $T \subseteq T_{G} \times T_{\Gamma}$. Put $S_{2}=$ $\left(T_{G} \cup S_{1, G}\right) \times\left\{e_{\Gamma}\right\} \in \overline{\mathcal{F}}(G \times \Gamma)$. Take an $\eta>0$ such that $\eta \Upsilon\left(S_{2}\right) \leq 1 / 3$. By Lemma 3.30 there is an $S_{\Gamma} \in \overline{\mathcal{F}}(\Gamma)$ such that for any free p.m.p. action $\Gamma \curvearrowright\left(Y, \mu_{Y}\right)$ there is a Borel set $\mathcal{V}_{Y} \subseteq Y$ so that $S_{\Gamma} \mathcal{V}_{Y}=Y$ and $\mu_{Y}\left(\mathcal{V}_{Y}\right) \leq \eta$.

Put $S_{3}=\left\{e_{G}\right\} \times\left(S_{\Gamma} T_{\Gamma} S_{\Gamma}\right) \in \overline{\mathcal{F}}(G \times \Gamma)$. Pick a $\delta>0$ such that $\delta \Upsilon\left(S_{3}\right) \leq 1 / 3$. Then we have $C_{1}$ as above. Put $C=4 C_{1}+3 \in \mathbb{N}$.

Let $G$ and $\Gamma$ act freely on standard Borel spaces $X$ and $Y$, respectively. Let $\mu$ be a $(G \times \Gamma)$ invariant Borel probability measure on $X \times Y$. Denote by $\mu_{X}$ and $\mu_{Y}$ the push-forward of $\mu$ under the projections $X \times Y \rightarrow X$ and $X \times Y \rightarrow Y$ respectively. Then we have $Z_{X}, \mathcal{V}_{X}$ and $\mathcal{V}_{Y}$ as above.

Put $\mathcal{V}_{1}=\mathcal{V}_{X} \times Y, \mathcal{V}_{2}=X \times \mathcal{V}_{Y}$, and $\mathcal{V}_{3}=Z_{X} \times Y$. Then

$$
\sum_{j=1}^{3} \Upsilon\left(S_{j}\right) \mu\left(\mathcal{V}_{j}\right)=\Upsilon\left(S_{1}\right) \mu_{X}\left(\mathcal{V}_{X}\right)+\Upsilon\left(S_{2}\right) \mu_{Y}\left(\mathcal{V}_{Y}\right)+\Upsilon\left(S_{3}\right) \mu_{X}\left(Z_{X}\right) \leq 1,
$$

verifying condition (i) in Definition 3.1 .

Put $W=\mathcal{V}_{X} \times Y$. Then $S W=X \times Y$, verifying condition (ii) in Definition 3.1 .

Let $t=\left(t_{G}, t_{\Gamma}\right) \in T$ and $w_{1}, w_{2} \in W$ with $t w_{1}=w_{2}$. Say, $w_{i}=\left(x_{i}, y_{i}\right)$ for $i=1,2$. Then $x_{i}$ is connected to some $z_{i} \in Z_{X}$ by an $S_{1, G}$-path of length at most $C_{1}$ with all points in $\mathcal{V}_{X}$. Put $w_{i}^{\prime}=\left(z_{i}, y_{i}\right)$. Then $w_{i}$ is connected to $w_{i}^{\prime}$ by an $S_{1}$-path of length at most $C_{1}$ with all points 
in $\mathcal{V}_{1}$. We have $s y_{1} \in \mathcal{V}_{Y}$ for some $s \in S_{\Gamma}$. Put $w_{1}^{\prime \prime}=\left(z_{1}, s y_{1}\right)$ and $w_{2}^{\prime \prime}=\left(z_{2}, s y_{1}\right)$. Note that $s y_{1}=s t_{\Gamma}^{-1} y_{2} \in S_{\Gamma} T_{\Gamma} S_{\Gamma} y_{2}$. Thus $\left(w_{i}^{\prime}, w_{i}^{\prime \prime}\right)$ is an $S_{3}$-edge with both endpoints in $\mathcal{V}_{3}$ for $i=1,2$. Also note that $z_{2} \in S_{1, G}^{C_{1}} x_{2} \subseteq S_{1, G}^{C_{1}} T_{G} x_{1} \subseteq S_{1, G}^{C_{1}} T_{G} S_{1, G}^{C_{1}} z_{1}$. Thus $w_{1}^{\prime \prime}$ and $w_{2}^{\prime \prime}$ are connected by an $S_{2}$-path of length at most $2 C_{1}+1$ with all points in $\mathcal{V}_{2}$. We conclude that $w_{1}$ and $w_{2}$ are connected by a path of length at most $4 C_{1}+3$ in which each edge is an $S_{j}$-edge with both endpoints in $\mathcal{V}_{j}$ for some $1 \leq j \leq 3$, verifying condition (iii) in Definition 3.1 ,

Gaboriau showed that if $R$ and $S$ are countable aperiodic Borel equivalence relations on standard Borel spaces $X$ and $Y$, respectively, and $\mu$ is an $(R \times S)$-invariant Borel probability measure on $X \times Y$, then the cost of $R \times S$ on $(X \times Y, \mu)$ is equal to 1 [15] [21, Theorem 24.9]. The following result is an analogue of Gaboriau's theorem.

Proposition 3.32. Let $G$ and $\Gamma$ be countably infinite groups. Then $G \times \Gamma$ has property $S C$ for product actions if and only if at least one of $G$ and $\Gamma$ is not locally finite.

Proof. If at least one of $G$ and $\Gamma$, say $G$, has the shrinking property, then $G \times \Gamma$ has property SC for product actions by Lemma 3.31 and $G$ is not locally finite by Proposition 3.11. Thus we may assume that neither $G$ nor $\Gamma$ has the shrinking property. By Proposition 3.11, each of $G$ and $\Gamma$ is either locally finite or virtually cyclic. If at least one of $G$ and $\Gamma$ is not locally finite, then $G \times \Gamma$ is neither locally finite nor virtually cyclic, and so $G \times \Gamma$, being amenable, has property SC by Proposition 3.28, If on the other hand $G$ and $\Gamma$ are both locally finite then $G \times \Gamma$ is locally finite, which implies by Theorem 3.20 that $G \times \Gamma$ has no free p.m.p. actions with property $\mathrm{SC}$ and hence does not itself have property $\mathrm{SC}$ for product actions.

\section{Measure entropy and Shannon orbit equivalence}

We devote ourselves in this section to the proof of the following theorem, which together with Theorem 3.29 yields Theorem A.

Theorem 4.1. Let $G$ and $H$ be countably infinite groups and let $G \curvearrowright(X, \mu)$ and $H \curvearrowright(Y, \nu)$ be free p.m.p. actions which are Shannon orbit equivalent. Suppose that $G \curvearrowright(X, \mu)$ has property $S C$. Then

$$
h_{\nu}(H \curvearrowright Y) \geq h_{\mu}(G \curvearrowright X) .
$$

For the purpose of proving the theorem we may assume, by conjugating the $H$-action by a Shannon orbit equivalence, that $(X, \mu)=(Y, \nu)$ and that the identity map from $X$ to itself provides a Shannon orbit equivalence between the two actions. As usual denote the associated cocycles $G \times X \rightarrow H$ and $H \times X \rightarrow G$ by $\kappa$ and $\lambda$, respectively.

For each $g \in G$ we write $\mathscr{P}_{g}$ for the countable Borel partition of $X$ consisting of the sets $X_{g, t}=\{x \in X: g x=t x\}$ for $t \in H$, and likewise for $t \in H$ we write $\mathscr{P}_{t}$ for the countable Borel partition of $X$ consisting of the sets $X_{g, t}$ for $g \in G$. For every $F$ in $\mathcal{F}(G)$ or $\mathcal{F}(H)$, set ${ }_{F} \mathscr{P}=\bigvee_{g \in F} \mathscr{P}_{g}$. Then $H_{\mu}\left({ }_{F} \mathscr{P}\right)<\infty$. For every $F \in \mathcal{F}(G)$ and $L \in \mathcal{F}(H)$, denote by ${ }_{F, L} \overline{\mathscr{P}}$ the finite set consisting of all $P \in{ }_{F} \mathscr{P}$ satisfying $\kappa(g, P) \in L$ for all $g \in F$ and set $X_{F, L}=\bigcup_{F, L} \overline{\mathscr{P}}$. Denote by ${ }_{F, L} \mathscr{P}$ the finite partition ${ }_{F, L} \overline{\mathscr{P}} \cup\left\{X \backslash X_{F, L}\right\}$ of $X$. Similarly, denote by ${ }_{L, F} \overline{\mathscr{P}}$ the finite set consisting of all $P \in{ }_{L} \mathscr{P}$ satisfying $\lambda(t, P) \in F$ for all $t \in L$, and set $X_{L, F}=\bigcup_{L, F} \overline{\mathscr{P}}$ and ${ }_{L, F} \mathscr{P}={ }_{L, F} \overline{\mathscr{P}} \cup\left\{X \backslash X_{L, F}\right\}$. 
Given a finite disjoint collection $\mathscr{C}$ of Borel subsets of $X$ and a nonempty finite set $V$, we define on the set of all maps with domain some collection of subsets of $X$ containing $\mathscr{C}$ and codomain $\mathbb{P}_{V}$ the pseudometric

$$
\rho_{\mathscr{C}}(\varphi, \psi)=\sum_{A \in \mathscr{C}} \mathrm{m}(\varphi(A) \Delta \psi(A))
$$

Lemma 4.2. Let $L \in \overline{\mathcal{F}}(H)$ and $0<\tau<1$. Take an $F^{\natural} \in \overline{\mathcal{F}}(G)$ such that $\mu\left(X_{L^{2}, F^{\natural}}\right) \geq 1-\tau / 30$, and take an $F \in \overline{\mathcal{F}}(G)$ such that $F^{\natural} \subseteq F$ and $\mu\left(X_{L^{2}, F}\right) \geq 1-\tau /\left(30\left|F^{\natural}\right|\right)$. Let $0<\tau^{\prime} \leq \tau /\left(60|F|^{2}\right)$. Let $\pi: G \rightarrow \operatorname{Sym}(V)$ be an $\left(F, \tau^{\prime}\right)$-approximation for $G$. Let $\varphi \in \operatorname{Hom}_{\mu}\left(L_{L^{2}, F} \mathscr{P}, F, \tau^{\prime}, \pi\right)$. Take $\sigma^{\prime}: L^{2} \rightarrow V^{V}$ such that

$$
\sigma_{t}^{\prime} v=\pi_{\lambda(t, A)} v
$$

for all $t \in L^{2}, A \in{ }_{L^{2}, F} \overline{\mathscr{P}}$ and $v \in \varphi(A)$. Then there is an $(L, \tau)$-approximation $\sigma: H \rightarrow$ $\operatorname{Sym}(V)$ for $H$ such that $\rho_{\text {Hamm }}\left(\sigma_{t}, \sigma_{t}^{\prime}\right) \leq \tau / 5$ for all $t \in L^{2}$.

Proof. Denote by $V_{F}$ the set of all $v \in V$ satisfying $\pi_{g} \pi_{h} v=\pi_{g h} v$ for all $g, h \in F$ and $\pi_{g} v \neq \pi_{h} v$ for all distinct $g, h \in F$. Then

Set

$$
\mathrm{m}\left(V \backslash V_{F}\right) \leq 2|F|^{2} \tau^{\prime} \leq \frac{\tau}{30}
$$

$$
V^{\prime}=\bigcup_{g \in F} \bigcup_{B \in_{L^{2}, F} \overline{\mathscr{P}}}\left(\varphi\left(g^{-1} B\right) \Delta \pi_{g^{-1}} \varphi(B)\right) \subseteq V .
$$

Then

Set $V^{*}=V_{F} \backslash V^{\prime}$. Then

$$
\mathrm{m}\left(V^{\prime}\right) \leq|F| \tau^{\prime} \leq \frac{\tau}{60}
$$

$$
\mathrm{m}\left(V^{*}\right) \geq \mathrm{m}\left(V_{F}\right)-\mathrm{m}\left(V^{\prime}\right) \geq 1-\frac{\tau}{30}-\frac{\tau}{60}=1-\frac{\tau}{20} .
$$

For each $t \in L^{2}$ set $V_{t}^{\sharp}=\bigsqcup_{A, B \in_{L^{2}, F} \overline{\mathscr{P}}} \varphi\left(A \cap \lambda(t, A)^{-1} B\right)$. Then

$$
\begin{aligned}
\mathrm{m}\left(V_{t}^{\sharp}\right) & \geq \mu\left(\bigsqcup_{A, B \in_{L^{2}, F} \overline{\mathscr{P}}}\left(A \cap \lambda(t, A)^{-1} B\right)\right)-\tau^{\prime} \\
& \geq \mu\left(\bigsqcup_{A \in_{L^{2}, F^{\natural}} \overline{\mathscr{P}}} \bigsqcup_{B \in_{L^{2}, F} \overline{\mathscr{P}}}\left(A \cap \lambda(t, A)^{-1} B\right)\right)-\tau^{\prime} \\
& \geq \mu\left(X_{L^{2}, F^{\natural}}\right)-\left|F^{\natural}\right|\left(1-\mu\left(X_{L^{2}, F}\right)\right)-\tau^{\prime} \\
& \geq 1-\frac{\tau}{30}-\frac{\tau}{30}-\frac{\tau}{60}=1-\frac{\tau}{12} .
\end{aligned}
$$

Let $s, t \in L^{2}$ with $s t \in L^{2}$. Let $v \in V^{*} \cap V_{t}^{\sharp}$. Then $v \in \varphi\left(A \cap \lambda(t, A)^{-1} B\right)=\varphi(A) \cap \varphi\left(g^{-1} B\right)$ for some $A, B \in{ }_{L^{2}, F} \overline{\mathscr{P}}$, where $g=\lambda(t, A) \in F$. Since $v \notin V^{\prime}$, we have $v \in \varphi(A) \cap \pi_{g^{-1}} \varphi(B)$. Using the fact that $v \in V_{F}$, we get $v \in \varphi(A) \cap \pi_{g}^{-1}(\varphi(B))$. Put $h=\lambda(s, B) \in F$. Note that $\sigma_{t}^{\prime} v=\pi_{g} v \in \varphi(B)$. Thus

$$
\sigma_{s}^{\prime} \sigma_{t}^{\prime} v=\sigma_{s}^{\prime} \pi_{g} v=\pi_{h} \pi_{g} v=\pi_{h g} v
$$


For every $x \in A \cap g^{-1} B$ we have $t x=g x \in B$ and hence

$$
\lambda(s t, x)=\lambda(s, t x) \lambda(t, x)=h g .
$$

Since $s t \in L^{2}$ and $A \in{ }_{L^{2}, F} \overline{\mathscr{P}}$, we have $h g=\lambda(s t, A) \in F$. Thus $\sigma_{s t}^{\prime} w=\pi_{h g} w$ for all $w \in \varphi\left(A \cap g^{-1} B\right)$, and so

$$
\sigma_{s}^{\prime} \sigma_{t}^{\prime} v=\pi_{h g} v=\sigma_{s t}^{\prime} v
$$

We conclude that

$$
\rho_{\text {Hamm }}\left(\sigma_{s}^{\prime} \sigma_{t}^{\prime}, \sigma_{s t}^{\prime}\right) \leq 1-\mathrm{m}\left(V^{*} \cap V_{t}^{\sharp}\right) \leq \frac{\tau}{20}+\frac{\tau}{12}=\frac{2 \tau}{15} .
$$

Note that $\sigma_{e_{H}}^{\prime}=\pi_{e_{G}}$ on $\varphi\left(X_{L^{2}, F}\right)$. Since $\pi$ is an $\left(F, \tau^{\prime}\right)$-approximation for $G$ we have $\rho_{\mathrm{Hamm}}\left(\pi_{e_{G}}, \mathrm{id}_{V}\right)=\rho_{\mathrm{Hamm}}\left(\pi_{e_{G}} \pi_{e_{G}}, \pi_{e_{G}}\right) \leq \tau^{\prime}$ and hence

$$
\begin{aligned}
\rho_{\mathrm{Hamm}}\left(\sigma_{e_{H}}^{\prime}, \operatorname{id}_{V}\right) & \leq \rho_{\mathrm{Hamm}}\left(\sigma_{e_{H}}^{\prime}, \pi_{e_{G}}\right)+\rho_{\mathrm{Hamm}}\left(\pi_{e_{G}}, \operatorname{id}_{V}\right) \\
& \leq 1-\operatorname{m}\left(\varphi\left(X_{L^{2}, F}\right)\right)+\tau^{\prime} \\
& \leq 1-\mu\left(X_{L^{2}, F}\right)+2 \tau^{\prime} \\
& \leq \frac{\tau}{30}+\frac{\tau}{30}=\frac{\tau}{15} .
\end{aligned}
$$

For each $t \in L^{2}$ pick a $\sigma_{t} \in \operatorname{Sym}(V)$ such that $\sigma_{t} v=\sigma_{t}^{\prime} v$ for all $v \in V$ satisfying $\sigma_{t^{-1}}^{\prime} \sigma_{t}^{\prime} v=v$. For each $t \in L^{2}$, taking $s=t^{-1}$ in (5) we conclude that

$$
\begin{aligned}
\rho_{\text {Hamm }}\left(\sigma_{t}, \sigma_{t}^{\prime}\right) & \leq \rho_{\text {Hamm }}\left(\sigma_{t^{-1}}^{\prime} \sigma_{t}^{\prime}, \operatorname{id}_{V}\right) \\
& \leq \rho_{\text {Hamm }}\left(\sigma_{t^{-1}}^{\prime} \sigma_{t}^{\prime}, \sigma_{e_{H}}^{\prime}\right)+\rho_{\text {Hamm }}\left(\sigma_{e_{H}}^{\prime}, \operatorname{id}_{V}\right) \\
& \leq \frac{2 \tau}{15}+\frac{\tau}{15}=\frac{\tau}{5} .
\end{aligned}
$$

For any $s, t \in L$ we then have

$$
\begin{aligned}
\rho_{\text {Hamm }}\left(\sigma_{s} \sigma_{t}, \sigma_{s t}\right) & \leq \rho_{\text {Hamm }}\left(\sigma_{s}, \sigma_{s}^{\prime}\right)+\rho_{\text {Hamm }}\left(\sigma_{t}, \sigma_{t}^{\prime}\right)+\rho_{\text {Hamm }}\left(\sigma_{s}^{\prime} \sigma_{t}^{\prime}, \sigma_{s t}^{\prime}\right)+\rho_{\text {Hamm }}\left(\sigma_{s t}, \sigma_{s t}^{\prime}\right) \\
& \leq \frac{\tau}{5}+\frac{\tau}{5}+\frac{2 \tau}{15}+\frac{\tau}{5}<\tau .
\end{aligned}
$$

Let $s, t \in L^{2}$ be distinct. Let $A \in{ }_{L^{2}, F} \overline{\mathscr{P}}$. Say, $g=\lambda(t, A) \in F$ and $h=\lambda(s, A) \in F$. Take $x \in A$. Then $g x=t x \neq s x=h x$, and hence $g \neq h$. Thus for any $v \in \varphi(A) \cap V_{F}$ we have $\sigma_{t}^{\prime} v=\pi_{g} v \neq \pi_{h} v=\sigma_{s}^{\prime} v$. This shows that $\sigma_{t}^{\prime} v \neq \sigma_{s}^{\prime} v$ for all $v \in V_{F} \cap \varphi\left(X_{L^{2}, F}\right)$. Therefore

$$
\begin{aligned}
\rho_{\text {Hamm }}\left(\sigma_{s}, \sigma_{t}\right) & \geq \rho_{\text {Hamm }}\left(\sigma_{s}^{\prime}, \sigma_{t}^{\prime}\right)-\rho_{\text {Hamm }}\left(\sigma_{s}, \sigma_{s}^{\prime}\right)-\rho_{\text {Hamm }}\left(\sigma_{t}, \sigma_{t}^{\prime}\right) \\
& \geq \mathrm{m}\left(V_{F} \cap \varphi\left(X_{L^{2}, F}\right)\right)-\frac{\tau}{5}-\frac{\tau}{5} \\
& \geq \mathrm{m}\left(\varphi\left(X_{L^{2}, F}\right)\right)-\frac{\tau}{30}-\frac{2 \tau}{5} \\
& \geq \mu\left(X_{L^{2}, F}\right)-\tau^{\prime}-\frac{13 \tau}{30} \\
& \geq 1-\frac{\tau}{30}-\frac{\tau}{60}-\frac{13 \tau}{30}>1-\tau .
\end{aligned}
$$


Lemma 4.3. Let $\mathscr{C}$ be a finite Borel partition of $X, L \in \overline{\mathcal{F}}(H)$, and $0<\tau<1$. Take an $F \in \overline{\mathcal{F}}(G)$ such that $\mu\left(X_{L^{2}, F}\right) \geq 1-\tau / 30$. Let $0<\tau^{\prime} \leq \tau /\left(60|F|^{2}\right)$. Let $\pi: G \rightarrow \operatorname{Sym}(V)$ be a sofic approximation for $G$. Let $\varphi \in \operatorname{Hom}_{\mu}\left(\mathscr{C}_{L} \vee{ }_{L^{2}, F} \mathscr{P}, F, \tau^{\prime}, \pi\right)$. Take a $\sigma^{\prime}: L^{2} \rightarrow V^{V}$ such that

$$
\sigma_{t}^{\prime} v=\pi_{\lambda(t, A)} v
$$

for all $t \in L^{2}, A \in{ }_{L^{2}, F} \overline{\mathscr{P}}$ and $v \in \varphi(A)$. Let $\sigma: H \rightarrow \operatorname{Sym}(V)$ be a sofic approximation for $H$ such that $\rho_{\text {Hamm }}\left(\sigma_{t}, \sigma_{t}^{\prime}\right) \leq \tau / 5$ for all $t \in L^{2}$. Then the restriction of $\varphi$ to $\operatorname{alg}\left(\mathscr{C}_{L}\right)$ lies in $\operatorname{Hom}_{\mu}(\mathscr{C}, L, \tau, \sigma)$.

Proof. We have

$$
\sum_{A \in \mathscr{C}_{L}}|\mathrm{~m}(\varphi(A))-\mu(A)| \leq \sum_{A \in\left(\mathscr{C}_{L} \vee_{L^{2}, F} \mathscr{P}\right)_{F}}|\mathrm{~m}(\varphi(A))-\mu(A)| \leq \tau^{\prime} \leq \tau,
$$

while for each $t \in L$ we have

$$
\begin{aligned}
& \sum_{A \in \mathscr{C}} \mathrm{m}(\varphi(\left.t A) \Delta \sigma_{t} \varphi(A)\right) \\
& \leq \sum_{A \in \mathscr{C}} \mathrm{m}\left(\varphi(t A) \Delta \sigma_{t}^{\prime} \varphi(A)\right)+\sum_{A \in \mathscr{C}} \mathrm{m}\left(\sigma_{t}^{\prime} \varphi(A) \Delta \sigma_{t} \varphi(A)\right) \\
& \leq \sum_{A \in \mathscr{C}} \sum_{B \in_{L^{2}, F} \overline{\mathscr{P}}} \mathrm{m}\left(\varphi(t(A \cap B)) \Delta \sigma_{t}^{\prime} \varphi(A \cap B)\right) \\
&+\sum_{A \in \mathscr{C}} \mathrm{m}\left(\varphi\left(t\left(A \backslash X_{L^{2}, F}\right)\right) \Delta \sigma_{t}^{\prime} \varphi\left(A \backslash X_{L^{2}, F}\right)\right)+2 \rho_{\text {Hamm }}\left(\sigma_{t}^{\prime}, \sigma_{t}\right) \\
& \leq \sum_{A \in \mathscr{C}} \sum_{B \in_{L^{2}, F} \bar{P}} \mathrm{~m}\left(\varphi(\lambda(t, B)(A \cap B)) \Delta \pi_{\lambda(t, B)} \varphi(A \cap B)\right) \\
&\left.\quad+\mathrm{m}_{(\varphi}\left(t\left(X \backslash X_{L^{2}, F}\right)\right)\right)+\mathrm{m}\left(\varphi\left(X \backslash X_{L^{2}, F}\right)\right)+\frac{2 \tau}{5} \\
& \leq \sum_{g \in F} \sum_{A \in \mathscr{C}} \sum_{B \in_{L^{2}, F}} \mathrm{~m}\left(\varphi(g(A \cap B)) \Delta \pi_{g} \varphi(A \cap B)\right) \\
& \quad+\mu\left(t\left(X \backslash X_{L^{2}, F}\right)\right)+\mu\left(X \backslash X_{L^{2}, F}\right)+2 \tau^{\prime}+\frac{2 \tau}{5} \\
& \leq|F| \tau^{\prime}+\frac{\tau}{15}+\frac{\tau}{30}+\frac{2 \tau}{5} \leq \frac{\tau}{60}+\frac{\tau}{2}<\tau .
\end{aligned}
$$

For a finite family $\mathscr{C}$ of Borel subsets of $X$, a finite Borel partition $\mathscr{P}$ of $X$, and $\tau \geq 0$ we write $\mathscr{C} \stackrel{\tau}{\subseteq} \operatorname{alg}(\mathscr{P})$ if there is a $B \in \operatorname{alg}(\mathscr{P})$ such that $\mu(B) \geq 1-\tau$ and $A \cap B \in \operatorname{alg}(\mathscr{P})$ for every $A \in \mathscr{C}$.

Lemma 4.4. Let $\mathscr{C}$ be a finite Borel partition of $X$. Let $F \in \mathcal{F}(G)$ and $L \in \mathcal{F}(H)$. Then $\mathscr{C}_{F} \stackrel{\tau}{\subseteq} \operatorname{alg}\left(\left(\mathscr{C} \vee_{F, L} \mathscr{P}\right)_{L}\right)$ for $\tau=\mu\left(X \backslash X_{F, L}\right)|F|$.

Proof. Put $B=\bigcap_{g \in F} g X_{F, L} \in \operatorname{alg}\left(\left(\mathscr{C} \vee_{F, L} \mathscr{P}\right)_{F}\right)$. Then $\mu(X \backslash B) \leq \tau$. 
Let $A \in\left(\mathscr{C} \vee_{F, L} \mathscr{P}\right)_{F}$. Then $A=\bigcap_{g \in F} g A_{g}$ for some $A_{g} \in \mathscr{C} \vee_{F, L} \mathscr{P}$. If $A_{g} \not \subset X_{F, L}$ for some $g \in F$, then $A \cap B=\emptyset$. If $A_{g} \subseteq X_{F, L}$ for all $g \in F$, then $A \subseteq B$ and $A=\bigcap_{g \in F} g A_{g}=$ $\bigcap_{g \in F} \kappa\left(g, A_{g}\right) A_{g} \in \operatorname{alg}\left(\left(\mathscr{C} \vee_{F, L} \mathscr{P}\right)_{L}\right)$. Thus

$$
\left(\mathscr{C} \vee_{F, L} \mathscr{P}\right)_{F} \stackrel{\tau}{\subseteq} \operatorname{alg}\left(\left(\mathscr{C} \vee_{F, L} \mathscr{P}\right)_{L}\right)
$$

Since $\mathscr{C}_{F} \subseteq \operatorname{alg}\left(\left(\mathscr{C} \vee_{F, L} \mathscr{P}\right)_{F}\right)$, we conclude that $\mathscr{C}_{F} \subseteq \stackrel{\tau}{\subseteq} \operatorname{alg}\left(\left(\mathscr{C} \vee_{F, L} \mathscr{P}\right)_{L}\right)$.

Lemma 4.5. Let $\mathscr{C}$ be a finite Borel partition of $X$. Let $S \in \overline{\mathcal{F}}(G)$ and let $W$ be a Borel subset of $X$ such that $S W=X$. Let $L$ be a set in $\mathcal{F}(H)$ containing $e_{H}$. Set $\mathscr{C}^{\prime}=\{W, X \backslash W\}$ and $\mathscr{C}^{\prime \prime}=\mathscr{C} \vee \mathscr{C}^{\prime} \vee{ }_{S^{2}, L} \mathscr{P}$. Then there are a finite Borel partition $\mathscr{Q}$ of $W$ contained in $\operatorname{alg}\left(\left(\mathscr{C} \vee \mathscr{C}^{\prime}\right)_{S^{2}}\right)$ and a map $\Theta: \mathscr{Q} \rightarrow \mathcal{F}(S)$ such that $e_{G} \in \Theta(B)$ for every $B \in \mathscr{Q}$ and the sets $g B$ for $B \in \mathscr{Q}$ and $g \in \Theta(B)$ form a partition $\mathscr{R}$ of $X$ finer than $\mathscr{C}$. Furthermore, for any such $\mathscr{Q}$ and $\mathscr{R}$ and any sofic approximations $\pi: G \rightarrow \operatorname{Sym}(V), \sigma: H \rightarrow \operatorname{Sym}(V)$, any $\delta, \delta^{\prime}>0$, any $\varphi, \psi \in \operatorname{Hom}_{\mu}\left(\mathscr{R}, S, \delta^{\prime}, \pi\right)$, and any $\tilde{\varphi}, \tilde{\psi} \in \operatorname{Hom}_{\mu}\left(\mathscr{R} \vee \mathscr{C}^{\prime \prime}, L, \delta, \sigma\right)$ satisfying

(i) $\tilde{\varphi}(B)=\varphi(B)$ and $\tilde{\psi}(B)=\psi(B)$ for all $B \in \mathscr{Q}$,

(ii) $\varphi(W)=\psi(W)$

one has

$$
\rho_{\mathscr{C}}(\varphi, \psi) \leq 2\left(\delta+\delta^{\prime}\right)|S|+2 \delta|S| \cdot|L|+2|S|^{3} \mu\left(X \backslash X_{S^{2}, L}\right)+|S| \cdot|L| \rho_{\mathscr{C} \vee_{S^{2}, L} \mathscr{P}}(\tilde{\varphi}, \tilde{\psi}) .
$$

Proof. We prove the existence of $\mathscr{Q}$ first. Note that $\left(\mathscr{C}^{\prime}\right)_{S}$ is the partition of $X$ generated by $g W$ for $g \in S$. Since $S W=X$, every member of $\left(\mathscr{C}^{\prime}\right)_{S}$ is contained in $g W$ for some $g \in S$. Each member $A$ of $\mathscr{C} \vee\left(\mathscr{C}^{\prime}\right)_{S}$ is contained in some member of $\left(\mathscr{C}^{\prime}\right)_{S}$, and hence is contained in $g_{A}^{-1} W$ for some $g_{A} \in S$. We shall choose $g_{A}=e_{G}$ when $A \subseteq W$. Denote by $\mathscr{Q}$ the partition of $W$ generated by $g_{A} A$ for $A \in \mathscr{C} \vee\left(\mathscr{C}^{\prime}\right)_{S}$. Then $\mathscr{Q} \subseteq \operatorname{alg}\left(\left(\mathscr{C} \vee\left(\mathscr{C}^{\prime}\right)_{S}\right)_{S}\right)$. Note that if $A$ is a member of $\mathscr{C} \vee\left(\mathscr{C}^{\prime}\right)_{S}$ then it can be written as $g_{A}^{-1}\left(g_{A} A\right)$ and hence is the disjoint union of sets of the form $g_{A}^{-1} B$ with $B$ belonging to $\mathscr{Q}$. Thus we can find a map $\Theta: \mathscr{Q} \rightarrow \mathcal{F}(S)$ such that $e_{G} \in \Theta(B)$ for every $B \in \mathscr{Q}$ and such that the sets $g B$ for $B \in \mathscr{Q}$ and $g \in \Theta(B)$ form a partition $\mathscr{R}$ of $X$ finer than $\mathscr{C} \vee\left(\mathscr{C}^{\prime}\right)_{S}$. Note that

$$
\left(\mathscr{C} \vee\left(\mathscr{C}^{\prime}\right)_{S}\right)_{S} \preceq\left(\left(\mathscr{C} \vee \mathscr{C}^{\prime}\right)_{S}\right)_{S}=\left(\mathscr{C} \vee \mathscr{C}^{\prime}\right)_{S^{2}}
$$

Thus

$$
\mathscr{Q} \subseteq \operatorname{alg}\left(\left(\mathscr{C} \vee\left(\mathscr{C}^{\prime}\right)_{S}\right)_{S}\right) \subseteq \operatorname{alg}\left(\left(\mathscr{C} \vee \mathscr{C}^{\prime}\right)_{S^{2}}\right) .
$$

Now let $\mathscr{Q}, \mathscr{R}, \pi, \sigma, \delta, \delta^{\prime}, \varphi, \psi, \tilde{\varphi}, \tilde{\psi}$ be as in the lemma statement. For any partitions $\mathscr{C}_{1}$ and $\mathscr{C}_{2}$ of $X$ coarser than $\left(\mathscr{R} \vee \mathscr{C}^{\prime \prime}\right)_{L}$, we have

$$
\begin{aligned}
\rho_{\mathscr{C}_{1} \vee \mathscr{C}_{2}}(\tilde{\varphi}, \tilde{\psi})= & \sum_{A_{1} \in \mathscr{C}_{1}, A_{2} \in \mathscr{C}_{2}} \mathrm{~m}\left(\left(\tilde{\varphi}\left(A_{1}\right) \cap \tilde{\varphi}\left(A_{2}\right)\right) \Delta\left(\tilde{\psi}\left(A_{1}\right) \cap \tilde{\psi}\left(A_{2}\right)\right)\right) \\
\leq & \sum_{A_{1} \in \mathscr{C}_{1}, A_{2} \in \mathscr{C}_{2}} \mathrm{~m}\left(\left(\tilde{\varphi}\left(A_{1}\right) \cap \tilde{\varphi}\left(A_{2}\right)\right) \Delta\left(\tilde{\psi}\left(A_{1}\right) \cap \tilde{\varphi}\left(A_{2}\right)\right)\right. \\
& \quad+\sum_{A_{1} \in \mathscr{C}_{1}, A_{2} \in \mathscr{C}_{2}} \mathrm{~m}\left(\left(\tilde{\psi}\left(A_{1}\right) \cap \tilde{\varphi}\left(A_{2}\right)\right) \Delta\left(\tilde{\psi}\left(A_{1}\right) \cap \tilde{\psi}\left(A_{2}\right)\right)\right) \\
& =\rho_{\mathscr{C}_{1}}(\tilde{\varphi}, \tilde{\psi})+\rho_{\mathscr{C}_{2}}(\tilde{\varphi}, \tilde{\psi}) .
\end{aligned}
$$


For each $t \in L$, we have

$$
\begin{aligned}
& \rho_{t \mathscr{C}^{\prime \prime}}(\tilde{\varphi}, \tilde{\psi})=\rho_{\mathscr{C}}{ }^{\prime \prime}(\tilde{\varphi} \circ t, \tilde{\psi} \circ t) \\
& \leq \rho_{\mathscr{C}}^{\prime \prime}\left(\tilde{\varphi} \circ t, \sigma_{t} \circ \tilde{\varphi}\right)+\rho_{\mathscr{C}}{ }^{\prime \prime}\left(\sigma_{t} \circ \tilde{\varphi}, \sigma_{t} \circ \tilde{\psi}\right)+\rho_{\mathscr{C}}{ }^{\prime \prime}\left(\sigma_{t} \circ \tilde{\psi}, \tilde{\psi} \circ t\right) \\
& \leq 2 \delta+\rho_{\mathscr{C}}{ }^{\prime \prime}(\tilde{\varphi}, \tilde{\psi})
\end{aligned}
$$

By Lemma 4.4 we have $\left(\mathscr{C} \vee \mathscr{C}^{\prime}\right)_{S^{2}} \stackrel{\tau}{\subseteq} \operatorname{alg}\left(\left(\mathscr{C}^{\prime \prime}\right)_{L}\right)$ for $\tau=\mu\left(X \backslash X_{S^{2}, L}\right)\left|S^{2}\right|$. Then $\mathscr{Q} \stackrel{\tau}{\subseteq} \operatorname{alg}\left(\left(\mathscr{C}^{\prime \prime}\right)_{L}\right)$. Thus

$$
\begin{aligned}
\rho_{\mathscr{Q}}(\tilde{\varphi}, \tilde{\psi}) & \leq \rho_{\left(\mathscr{C}^{\prime \prime}\right)}(\tilde{\varphi}, \tilde{\psi})+2 \tau+2 \delta \\
& \leq \sum_{t \in L} \rho_{t \mathscr{C}^{\prime \prime}}(\tilde{\varphi}, \tilde{\psi})+2 \tau+2 \delta \\
& \stackrel{(6)}{\leq} 2 \delta|L|+|L| \rho_{\mathscr{C}}{ }^{\prime \prime}(\tilde{\varphi}, \tilde{\psi})+2 \tau+2 \delta \\
& \leq 2 \delta|L|+|L| \rho_{\mathscr{C} \vee_{S^{2}, L} \mathscr{P}}(\tilde{\varphi}, \tilde{\psi})+|L| \rho_{\mathscr{C}^{\prime}}(\tilde{\varphi}, \tilde{\psi})+2 \tau+2 \delta \\
& =2 \delta|L|+|L| \rho_{\mathscr{C} \vee_{S^{2}, L} \mathscr{P}}(\tilde{\varphi}, \tilde{\psi})+2 \tau+2 \delta .
\end{aligned}
$$

As in (므), for each $g \in S$ we have

$$
\rho_{g \mathscr{Q}}(\varphi, \psi) \leq 2 \delta^{\prime}+\rho_{\mathscr{Q}}(\varphi, \psi)
$$

Thus

$$
\begin{aligned}
\rho_{\mathscr{C}}(\varphi, \psi) & \leq \rho_{\mathscr{R}}(\varphi, \psi) \\
& \leq \sum_{g \in S} \rho_{g \mathscr{Q}}(\varphi, \psi) \\
& \leq 2 \delta^{\prime}|S|+|S| \rho_{\mathscr{Q}}(\varphi, \psi) \\
& =2 \delta^{\prime}|S|+|S| \rho_{\mathscr{Q}}(\tilde{\varphi}, \tilde{\psi}) \\
& \leq 2 \delta^{\prime}|S|+2 \delta|S| \cdot|L|+|S| \cdot|L| \rho_{\mathscr{C} \vee_{S^{2}, L} \mathscr{P}}(\tilde{\varphi}, \tilde{\psi})+2|S| \tau+2|S| \delta \\
& \leq 2\left(\delta+\delta^{\prime}\right)|S|+2 \delta|S| \cdot|L|+2|S|^{3} \mu\left(X \backslash X_{S^{2}, L}\right)+|S| \cdot|L| \rho_{\mathscr{C} \vee_{S^{2}, L} \mathscr{P}}(\tilde{\varphi}, \tilde{\psi}) .
\end{aligned}
$$

Lemma 4.6. Let $S$ be a set in $\mathcal{F}(G)$ containing $e_{G}$ and let $W$ be a Borel subset of $X$ such that $S W=X$. Let $\mathscr{Q}$ be a finite Borel partition of $W$ and $\Theta$ a function $\mathscr{Q} \rightarrow \mathcal{F}(S)$ such that $e_{G} \in \Theta(B)$ for every $B \in \mathscr{Q}$ and such that the sets $g B$ for $B \in \mathscr{Q}$ and $g \in \Theta(B)$ form a partition of $X$. Also, let $\mathscr{D}$ be a finite Borel partition of $X$. Then there is a finite Borel partition $\mathscr{Q}_{1}$ of $W$ satisfying the following conditions:

(i) $\mathscr{Q} \preceq \mathscr{Q}_{1}$,

(ii) defining $\Theta(D)=\Theta(B)$ for $D \in \mathscr{Q}_{1}$ and $B \in \mathscr{Q}$ satisfying $D \subseteq B$ and denoting by $\mathscr{R}_{1}$ the partition of $X$ consisting of $g D$ for $D \in \mathscr{Q}_{1}$ and $g \in \Theta(D)$, one has $\mathscr{D} \preceq \mathscr{R}_{1}$.

Proof. Denote by $\mathscr{Q}_{1}$ the partition of $W$ generated by the sets $B \cap g^{-1} D$ for $B \in \mathscr{Q}, g \in \Theta(B)$, and $D \in \mathscr{D}$. It is easily checked that $\mathscr{Q}_{1}$ satisfies the conditions.

Lemma 4.7. Let $\mathscr{C}$ be a Borel finite partition of $X, L \in \overline{\mathcal{F}}(H)$, and $0<\delta<1$. Let $S \in$ $\overline{\mathcal{F}}(G)$ and let $W$ be a Borel subset of $X$ such that $S W=X$. Take an $L^{\bullet} \in \overline{\mathcal{F}}(H)$ such that 
$\mu\left(X \backslash X_{S, L} \bullet\right) \leq \delta /(20|S|)$ and an $\bar{L} \in \overline{\mathcal{F}}(H)$ containing $L^{\bullet}$. Let $\mathscr{Q}_{1} \preceq \mathscr{Q}_{2}$ be finite Borel partitions of $W$ and let $\Theta: \mathscr{Q}_{1} \cup \mathscr{Q}_{2} \rightarrow \mathcal{F}(S)$ be such that

(i) $e_{G} \in \Theta\left(B_{2}\right)=\Theta\left(B_{1}\right)$ for all $B_{2} \in \mathscr{Q}_{2}$ and $B_{1} \in \mathscr{Q}_{1}$ with $B_{2} \subseteq B_{1}$,

(ii) for $j=1,2$ the sets $g B$ for $B \in \mathscr{Q}_{j}$ and $g \in \Theta(B)$ form a partition $\mathscr{R}_{j}$ of $X$,

(iii) $\mathscr{R}_{1} \succeq \mathscr{D}:=\mathscr{C}_{L} \vee_{S, \bar{L}} \mathscr{P}$, and

(iv) $\mathscr{R}_{2} \succeq\left(\mathscr{R}_{1}\right)_{L \bar{L}}$.

Denote by $\mathscr{Q}_{1}^{\prime}$ the set of all $B \in \mathscr{Q}_{1}$ satisfying $B \subseteq X_{S, L} \bullet$ and by $\mathscr{Q}_{2}^{\prime}$ the set of all $B \in \mathscr{Q}_{2}$ satisfying $B \subseteq X_{S, \bar{L}}$. Denote by $\Lambda$ the set consisting of all $(B, g)$ for $B \in \mathscr{Q}_{2}^{\prime}$ and $g \in \Theta(B) \backslash\left\{e_{G}\right\}$. Take $0<\delta^{\prime} \leq \delta /(20|S|)$. Let $\mathscr{A}$ be a finite Borel partition of $X$ refining $\mathscr{R}_{2}$. Let $V$ be a nonempty finite set and $\varphi$ a homomorphism $\operatorname{alg}(\mathscr{A}) \rightarrow \mathbb{P}_{V}$ such that $\sum_{A \in \mathscr{A}}|\mathrm{m}(\varphi(A))-\mu(A)| \leq \delta^{\prime}$. Let $0<\bar{\tau} \leq \delta /\left(20\left|L^{\bullet}\right|\right)$. Let $\sigma: H \rightarrow \operatorname{Sym}(V)$ be an $\left(L \cup L^{\bullet}, \bar{\tau}\right)$-approximation for $H$. Define a map $\tilde{\varphi}^{\prime}: \mathscr{R}_{2} \rightarrow \mathbb{P}_{V}$ by $\tilde{\varphi}^{\prime}(B)=\varphi(B)$ for all $B \in \mathscr{Q}_{2}, \tilde{\varphi}^{\prime}(g B)=\emptyset$ for all $B \in \mathscr{Q}_{2} \backslash \mathscr{Q}_{2}^{\prime}$ and $g \in \Theta(B) \backslash\left\{e_{G}\right\}$, and

$$
\tilde{\varphi}^{\prime}(g B)=\sigma_{\kappa(g, B)} \varphi(B)
$$

for all $(B, g) \in \Lambda$, and extend $\tilde{\varphi}^{\prime}$ to a map alg $\left(\mathscr{R}_{2}\right) \rightarrow \mathbb{P}_{V}$ by setting $\tilde{\varphi}^{\prime}(D)=\bigcup_{A \in \mathscr{R}_{2}, A \subseteq D} \tilde{\varphi}^{\prime}(A)$ for $D \in \operatorname{alg}\left(\mathscr{R}_{2}\right)$. Suppose that

$$
\sum_{(B, g) \in \Lambda} \mathrm{m}\left(\tilde{\varphi}^{\prime}(g B) \cap \varphi(W)\right) \leq \frac{\delta}{40},
$$

$$
\sum_{(B, g),\left(B^{\prime}, g^{\prime}\right) \in \Lambda,(B, g) \neq\left(B^{\prime}, g^{\prime}\right)} \mathrm{m}\left(\tilde{\varphi}^{\prime}(g B) \cap \tilde{\varphi}^{\prime}\left(g^{\prime} B^{\prime}\right)\right) \leq \frac{\delta}{40},
$$

and

$$
\sum_{B \in \mathscr{Q}_{1}^{\prime}} \sum_{t \in L L} \mathrm{~m}\left(\tilde{\varphi}^{\prime}(t B) \Delta \sigma_{t} \varphi(B)\right) \leq \frac{\delta}{20} .
$$

Then there is a homomorphism $\tilde{\varphi}: \operatorname{alg}\left(\mathscr{R}_{2}\right) \rightarrow \mathbb{P}_{V}$ such that $\tilde{\varphi}(B)=\varphi(B)$ for every $B \in \mathscr{Q}_{2}$ and $\sum_{A \in \mathscr{R}_{2}} \mathrm{~m}\left(\tilde{\varphi}(A) \Delta \tilde{\varphi}^{\prime}(A)\right) \leq \delta / 5$. Furthermore, the restriction of any such $\tilde{\varphi}$ to $\operatorname{alg}\left(\mathscr{C}_{L}\right)$ lies in $\operatorname{Hom}_{\mu}(\mathscr{C}, L, \delta, \sigma)$.

Proof. Note that

$$
\begin{aligned}
\mu\left(X \backslash\left(W \cup \bigcup_{(B, g) \in \Lambda} g B\right)\right) & =\mu\left(\bigcup_{B \in \mathscr{Q}_{2} \backslash \mathscr{Q}_{2}^{\prime}} \bigcup_{g \in \Theta(B) \backslash\left\{e_{G}\right\}} g B\right) \\
& \leq(|S|-1) \mu\left(X \backslash X_{S, L} \bullet\right) \leq \frac{\delta}{20} .
\end{aligned}
$$

We prove the existence of $\tilde{\varphi}$ first. If $W=X$, then we may take $\tilde{\varphi}(A)=\varphi(A)$ for all $A \in \operatorname{alg}\left(\mathscr{R}_{2}\right)$. Thus we may assume that $W \neq X$. Consider the case that $\Lambda$ is nonempty. List the elements of $\Lambda$ as $\left(B_{1}, g_{1}\right), \ldots,\left(B_{|\Lambda|}, g_{|\Lambda|}\right)$. We set $\tilde{\varphi}(B)=\varphi(B)$ for all $B \in \mathscr{Q}_{2}, \tilde{\varphi}(g B)=\emptyset$ for all $B \in \mathscr{Q}_{2} \backslash \mathscr{Q}_{2}^{\prime}$ and $g \in \Theta(B) \backslash\left\{e_{G}\right\}$,

$$
\tilde{\varphi}\left(g_{k} B_{k}\right)=\tilde{\varphi}^{\prime}\left(g_{k} B_{k}\right) \backslash\left(\varphi(W) \cup \bigcup_{j=1}^{k-1} \tilde{\varphi}^{\prime}\left(g_{j} B_{j}\right)\right)
$$


for all $1 \leq k<|\Lambda|$, and

$$
\tilde{\varphi}\left(g_{|\Lambda|} B_{|\Lambda|}\right)=V \backslash\left(\varphi(W) \cup \bigcup_{j=1}^{|\Lambda|-1} \tilde{\varphi}^{\prime}\left(g_{j} B_{j}\right)\right) .
$$

Then the sets $\tilde{\varphi}(A)$ for $A \in \mathscr{R}_{2}$ form a partition of $V$, and hence $\tilde{\varphi}$ extends uniquely to a homomorphism $\operatorname{alg}\left(\mathscr{R}_{2}\right) \rightarrow \mathbb{P}_{V}$. We have

$$
\begin{aligned}
\mathrm{m}(\varphi(W))+\sum_{j=1}^{|\Lambda|} \mathrm{m}\left(\tilde{\varphi}^{\prime}\left(g_{j} B_{j}\right)\right) & =\mathrm{m}(\varphi(W))+\sum_{j=1}^{|\Lambda|} \mathrm{m}\left(\varphi\left(B_{j}\right)\right) \\
& =\mathrm{m}(\varphi(W))+\sum_{g \in S \backslash\left\{e_{G}\right\}} \sum_{1 \leq j \leq|\Lambda|, g_{j}=g} \mathrm{~m}\left(\varphi\left(B_{j}\right)\right) \\
& =\mathrm{m}(\varphi(W))+\sum_{g \in S \backslash\left\{e_{G}\right\}} \mathrm{m}\left(\varphi\left(\bigcup_{1 \leq j \leq|\Lambda|, g_{j}=g} B_{j}\right)\right) \\
& \geq \mu(W)-\delta^{\prime}+\sum_{g \in S \backslash\left\{e_{G}\right\}}\left(\mu\left(\bigcup_{1 \leq j \leq|\Lambda|, g_{j}=g} B_{j}\right)-\delta^{\prime}\right) \\
& =\mu\left(W \cup \bigcup_{(B, g) \in \Lambda} g B\right)-|S| \delta^{\prime} \\
& \stackrel{(10)}{\geq} 1-\frac{\delta}{20}-|S| \delta^{\prime} \geq 1-\frac{\delta}{10}
\end{aligned}
$$

and hence

$$
\begin{aligned}
\mathrm{m}(V \backslash(\varphi(W) & \left.\left.\cup \bigcup_{j=1}^{|\Lambda|} \tilde{\varphi}^{\prime}\left(g_{j} B_{j}\right)\right)\right) \\
& =1-\mathrm{m}\left(\varphi(W) \cup \bigcup_{j=1}^{|\Lambda|} \tilde{\varphi}^{\prime}\left(g_{j} B_{j}\right)\right) \\
& \leq \frac{\delta}{10}+\mathrm{m}(\varphi(W))+\sum_{j=1}^{|\Lambda|} \mathrm{m}\left(\tilde{\varphi}^{\prime}\left(g_{j} B_{j}\right)\right)-\mathrm{m}\left(\varphi(W) \cup \bigcup_{j=1}^{|\Lambda|} \tilde{\varphi}^{\prime}\left(g_{j} B_{j}\right)\right) \\
& =\frac{\delta}{10}+\sum_{k=1}^{|\Lambda|} \mathrm{m}\left(\tilde{\varphi}^{\prime}\left(g_{k} B_{k}\right) \cap\left(\varphi(W) \cup \bigcup_{j=1}^{k-1} \tilde{\varphi}^{\prime}\left(g_{j} B_{j}\right)\right)\right) .
\end{aligned}
$$

Therefore

$$
\begin{aligned}
\sum_{A \in \mathscr{R}_{2}} \mathrm{~m}\left(\tilde{\varphi}(A) \Delta \tilde{\varphi}^{\prime}(A)\right) & =\sum_{k=1}^{|\Lambda|} \mathrm{m}\left(\tilde{\varphi}\left(g_{k} B_{k}\right) \Delta \tilde{\varphi}^{\prime}\left(g_{k} B_{k}\right)\right) \\
& =\mathrm{m}\left(V \backslash\left(\varphi(W) \cup \bigcup_{j=1}^{|\Lambda|} \tilde{\varphi}^{\prime}\left(g_{j} B_{j}\right)\right)\right)
\end{aligned}
$$




$$
\begin{aligned}
& +\sum_{k=1}^{|\Lambda|} \mathrm{m}\left(\tilde{\varphi}^{\prime}\left(g_{k} B_{k}\right) \cap\left(\varphi(W) \cup \bigcup_{j=1}^{k-1} \tilde{\varphi}^{\prime}\left(g_{j} B_{j}\right)\right)\right) \\
& \leq \frac{\delta}{10}+2 \sum_{k=1}^{|\Lambda|} \mathrm{m}\left(\tilde{\varphi}^{\prime}\left(g_{k} B_{k}\right) \cap\left(\varphi(W) \cup \bigcup_{j=1}^{k-1} \tilde{\varphi}^{\prime}\left(g_{j} B_{j}\right)\right)\right) \\
& \leq \frac{\delta}{10}+2 \sum_{k=1}^{|\Lambda|} \mathrm{m}\left(\tilde{\varphi}^{\prime}\left(g_{k} B_{k}\right) \cap \varphi(W)\right) \\
& +2 \sum_{1 \leq j<k \leq|\Lambda|} \mathrm{m}\left(\tilde{\varphi}^{\prime}\left(g_{k} B_{k}\right) \cap \tilde{\varphi}^{\prime}\left(g_{j} B_{j}\right)\right) \\
& \stackrel{\text { (77), (8) }}{\leq} \frac{\delta}{10}+\frac{\delta}{20}+\frac{\delta}{20}=\frac{\delta}{5} \text {. }
\end{aligned}
$$

This proves the existence of $\tilde{\varphi}$ when $\Lambda$ is nonempty. Next consider the case that $W \neq X$ and $\Lambda=\emptyset$. Choose a $B_{0} \in \mathscr{Q}_{2} \backslash \mathscr{Q}_{2}^{\prime}$ and a $g_{0} \in \Phi(B) \backslash\left\{e_{G}\right\}$. Set $\tilde{\varphi}(B)=\varphi(B)$ for all $B \in \mathscr{Q}_{2}$, $\tilde{\varphi}\left(g_{0} B_{0}\right)=V \backslash \varphi(W)$, and $\tilde{\varphi}(g B)=\emptyset$ for all $B \in \mathscr{Q}_{2} \backslash \mathscr{Q}_{2}^{\prime}$ and $g \in \Phi(B) \backslash\left\{e_{G}\right\}$ satisfying $(B, g) \neq\left(B_{0}, g_{0}\right)$. Then the sets $\tilde{\varphi}(A)$ for $A \in \mathscr{R}_{2}$ form a partition of $V$, and hence $\tilde{\varphi}$ extends uniquely to a homomorphism $\operatorname{alg}\left(\mathscr{R}_{2}\right) \rightarrow \mathbb{P}_{V}$. We have

$$
\begin{aligned}
\sum_{A \in \mathscr{R}_{2}} \mathrm{~m}\left(\tilde{\varphi}(A) \Delta \tilde{\varphi}^{\prime}(A)\right) & =\mathrm{m}\left(\tilde{\varphi}\left(g_{0} B_{0}\right)\right) \\
& =\mathrm{m}(V \backslash \varphi(W)) \\
& \leq \mu(X \backslash W)+\delta^{\prime} \\
& \stackrel{(10)}{\leq} \frac{\delta}{20}+\frac{\delta}{20}<\frac{\delta}{5} .
\end{aligned}
$$

This proves the existence of $\tilde{\varphi}$.

Now let $\tilde{\varphi}$ be any homomorphism $\operatorname{alg}\left(\mathscr{R}_{2}\right) \rightarrow \mathbb{P}_{V}$ satisfying $\sum_{A \in \mathscr{R}_{2}} \mathrm{~m}\left(\tilde{\varphi}(A) \Delta \tilde{\varphi}^{\prime}(A)\right) \leq \delta / 5$. We have

$$
\begin{aligned}
\sum_{A \in \mathscr{R}_{2}} \mid & \mathrm{m}\left(\tilde{\varphi}^{\prime}(A)\right)-\mu(A) \mid \\
= & \sum_{B \in \mathscr{Q}_{2}, g \in \Theta(B)}\left|\mathrm{m}\left(\tilde{\varphi}^{\prime}(g B)\right)-\mu(g B)\right| \\
& \leq \sum_{B \in \mathscr{Q}_{2}}|\mathrm{~m}(\varphi(B))-\mu(B)|+\sum_{(B, g) \in \Lambda}|\mathrm{m}(\varphi(B))-\mu(B)|+\sum_{\substack{B \in \mathscr{Q}_{2} \backslash \mathscr{Q}_{2}^{\prime}, g \in \Theta(B) \backslash\left\{e_{G}\right\}}} \mu(B) \\
& \leq \delta^{\prime}+\sum_{g \in S \backslash\left\{e_{G}\right\}} \sum_{1 \leq j \leq|\Lambda|, g_{j}=g}\left|\mathrm{~m}\left(\varphi\left(B_{j}\right)\right)-\mu\left(B_{j}\right)\right|+(|S|-1) \mu\left(X \backslash X_{S, L}\right) \\
& \leq|S| \delta^{\prime}+\frac{\delta}{20} \leq \frac{\delta}{20}+\frac{\delta}{20}=\frac{\delta}{10},
\end{aligned}
$$


and hence

$$
\begin{aligned}
\sum_{A \in \mathscr{C}_{L}}|\mathrm{~m}(\tilde{\varphi}(A))-\mu(A)| & \leq \sum_{A \in \mathscr{R}_{2}}|\mathrm{~m}(\tilde{\varphi}(A))-\mu(A)| \\
& \leq \sum_{A \in \mathscr{R}_{2}}\left|\mathrm{~m}(\tilde{\varphi}(A))-\mathrm{m}\left(\tilde{\varphi}^{\prime}(A)\right)\right|+\sum_{A \in \mathscr{R}_{2}}\left|\mathrm{~m}\left(\tilde{\varphi}^{\prime}(A)\right)-\mu(A)\right| \\
& \stackrel{(111)}{\leq} \sum_{A \in \mathscr{R}_{2}} \mathrm{~m}\left(\tilde{\varphi}(A) \Delta \tilde{\varphi}^{\prime}(A)\right)+\frac{\delta}{10} \leq \frac{\delta}{5}+\frac{\delta}{10}<\delta .
\end{aligned}
$$

Let $t \in L$. Since $\mathscr{R}_{1}$ refines $\mathscr{C}_{L}$, it refines $\mathscr{C}$ and $t \mathscr{C}$. Thus

$$
\sum_{A \in \mathscr{C}} \mathrm{m}\left(\tilde{\varphi}(A) \Delta \tilde{\varphi}^{\prime}(A)\right) \leq \sum_{A \in \mathscr{R}_{2}} \mathrm{~m}\left(\tilde{\varphi}(A) \Delta \tilde{\varphi}^{\prime}(A)\right) \leq \frac{\delta}{5}
$$

and

$$
\sum_{A \in \mathscr{C}} \mathrm{m}\left(\tilde{\varphi}(t A) \Delta \tilde{\varphi}^{\prime}(t A)\right) \leq \sum_{A \in \mathscr{R}_{2}} \mathrm{~m}\left(\tilde{\varphi}(A) \Delta \tilde{\varphi}^{\prime}(A)\right) \leq \frac{\delta}{5} .
$$

For every $B \in \mathscr{Q}_{1}^{\prime}$ and $g \in \Theta(B) \backslash\left\{e_{G}\right\}$, we have

$$
\begin{aligned}
\tilde{\varphi}^{\prime}(g B)=\tilde{\varphi}^{\prime}\left(\bigcup_{B^{\prime} \in \mathscr{Q}_{2}^{\prime}, B^{\prime} \subseteq B} g B^{\prime}\right) & =\bigcup_{B^{\prime} \in \mathscr{Q}_{2}^{\prime}, B^{\prime} \subseteq B} \tilde{\varphi}^{\prime}\left(g B^{\prime}\right) \\
& =\bigcup_{B^{\prime} \in \mathscr{Q}_{2}^{\prime}, B^{\prime} \subseteq B} \sigma_{\kappa(g, B)} \varphi\left(B^{\prime}\right) \\
& =\sigma_{\kappa(g, B)} \varphi(B) .
\end{aligned}
$$

Note that

$$
\begin{aligned}
& \sum_{\substack{B \in \mathscr{Q}_{1} \backslash \mathscr{Q}_{1}^{\prime}, g \in \Theta(B)}} \mathrm{m}\left(\tilde{\varphi}^{\prime}(\operatorname{tg} B) \Delta \sigma_{t} \tilde{\varphi}^{\prime}(g B)\right) \\
& \leq \sum_{\substack{B \in \mathscr{Q}_{1} \backslash \mathscr{Q}_{1}^{\prime}, g \in \Theta(B)}} \mathrm{m}\left(\tilde{\varphi}^{\prime}(\operatorname{tg} B)\right)+\sum_{\substack{B \in \mathscr{Q}_{1} \backslash \mathscr{Q}_{1}^{\prime} \\
\mathrm{m}}} \mathrm{m}(\varphi(B))+\sum_{\substack{B \in \mathscr{Q}_{1} \backslash \mathscr{Q}_{1}^{\prime}, B \subseteq X_{S, \bar{L}}, g \in \Theta(B) \backslash\left\{e_{G}\right\}}} \mathrm{m}\left(\sigma_{\kappa(g, B)} \varphi(B)\right)
\end{aligned}
$$

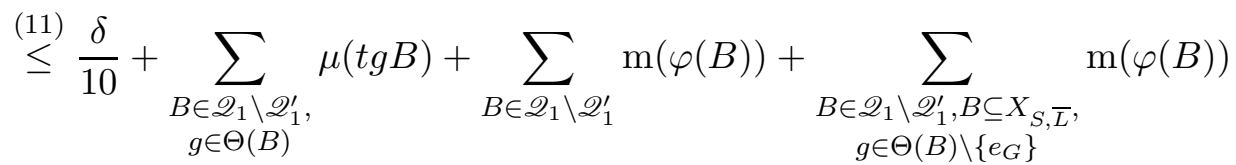

$$
\begin{aligned}
& \leq \frac{\delta}{10}+|S| \mu\left(X \backslash X_{S, L} \bullet\right)+|S| \mathrm{m}\left(\varphi\left(X \backslash X_{S, L} \bullet\right)\right) \\
& \leq \frac{\delta}{10}+|S| \mu\left(X \backslash X_{S, L} \bullet\right)+|S| \mu\left(X \backslash X_{S, L} \bullet\right)+|S| \delta^{\prime} \\
& \leq \frac{\delta}{10}+\frac{\delta}{20}+\frac{\delta}{20}+\frac{\delta}{20}=\frac{\delta}{4} \text {. }
\end{aligned}
$$


Thus

$$
\begin{aligned}
& \sum_{A \in \mathscr{C}} \mathrm{m}\left(\tilde{\varphi}^{\prime}(t A) \Delta \sigma_{t} \tilde{\varphi}^{\prime}(A)\right) \\
& =\sum_{A \in \mathscr{C}} \mathrm{m}\left(\left(\bigcup_{D \in \mathscr{R}_{1}, D \subseteq A} \tilde{\varphi}^{\prime}(t D)\right) \Delta\left(\bigcup_{D \in \mathscr{R}_{1}, D \subseteq A} \sigma_{t} \tilde{\varphi}^{\prime}(D)\right)\right) \\
& \leq \sum_{A \in \mathscr{C}} \sum_{D \in \mathscr{R}_{1}, D \subseteq A} \mathrm{~m}\left(\tilde{\varphi}^{\prime}(t D) \Delta \sigma_{t} \tilde{\varphi}^{\prime}(D)\right) \\
& =\sum_{D \in \mathscr{R}_{1}} \mathrm{~m}\left(\tilde{\varphi}^{\prime}(t D) \Delta \sigma_{t} \tilde{\varphi}^{\prime}(D)\right) \\
& =\sum_{B \in \mathscr{Q}_{1}, g \in \Theta(B)} \mathrm{m}\left(\tilde{\varphi}^{\prime}(t g B) \Delta \sigma_{t} \tilde{\varphi}^{\prime}(g B)\right) \\
& =\sum_{B \in \mathscr{Q}_{1}^{\prime}, g \in \Theta(B)} \mathrm{m}\left(\tilde{\varphi}^{\prime}(t \kappa(g, B) B) \Delta \sigma_{t} \tilde{\varphi}^{\prime}(g B)\right)+\sum_{\substack{B \in \mathscr{Q}_{1} \backslash \mathscr{Q}_{1}^{\prime}, g \in \Theta(B)}} \mathrm{m}\left(\tilde{\varphi}^{\prime}(t g B) \Delta \sigma_{t} \tilde{\varphi}^{\prime}(g B)\right) \\
& \stackrel{\text { (15) }}{\leq} \sum_{B \in \mathscr{Q}_{1}^{\prime}, g \in \Theta(B)} \mathrm{m}\left(\tilde{\varphi}^{\prime}(t \kappa(g, B) B) \Delta \sigma_{t \kappa(g, B)} \varphi(B)\right) \\
& +\sum_{B \in \mathscr{Q}_{1}^{\prime}, g \in \Theta(B)} \mathrm{m}\left(\sigma_{t \kappa(g, B)} \varphi(B) \Delta \sigma_{t} \sigma_{\kappa(g, B)} \varphi(B)\right) \\
& +\sum_{B \in \mathscr{Q}_{1}^{\prime}, g \in \Theta(B)} \mathrm{m}\left(\sigma_{t} \sigma_{\kappa(g, B)} \varphi(B) \Delta \sigma_{t} \tilde{\varphi}^{\prime}(g B)\right)+\frac{\delta}{4} \\
& \stackrel{\text { (14) }}{\leq} \sum_{B \in \mathscr{Q}_{1}^{\prime}, t_{1} \in L L} \operatorname{m}\left(\tilde{\varphi}^{\prime}\left(t_{1} B\right) \Delta \sigma_{t_{1}} \varphi(B)\right)+2 \sum_{t_{1} \in L} \rho_{\text {Hamm }}\left(\sigma_{t t_{1}}, \sigma_{t} \sigma_{t_{1}}\right) \\
& +\sum_{B \in \mathscr{Q}_{1}^{\prime}} \mathrm{m}\left(\sigma_{t} \sigma_{e_{H}} \varphi(B) \Delta \sigma_{t} \varphi(B)\right)+\frac{\delta}{4} \\
& \stackrel{\text { (9) }}{\leq} \frac{\delta}{20}+2 \bar{\tau}\left|L^{\bullet}\right|+\sum_{B \in \mathscr{Q}_{1}^{\prime}} \mathrm{m}\left(\sigma_{e_{H}} \varphi(B) \Delta \varphi(B)\right)+\frac{\delta}{4} \\
& \leq \frac{\delta}{20}+2 \bar{\tau}\left|L^{\bullet}\right|+2 \rho_{\mathrm{Hamm}}\left(\sigma_{e_{H}}, \mathrm{id}_{V}\right)+\frac{\delta}{4} \\
& =\frac{\delta}{20}+2 \bar{\tau}\left|L^{\bullet}\right|+2 \rho_{\mathrm{Hamm}}\left(\sigma_{e_{H}} \sigma_{e_{H}}, \sigma_{e_{H}}\right)+\frac{\delta}{4} \\
& \leq \frac{\delta}{20}+4 \bar{\tau}\left|L^{\bullet}\right|+\frac{\delta}{4} \leq \frac{\delta}{20}+\frac{\delta}{5}+\frac{\delta}{4}=\frac{\delta}{2},
\end{aligned}
$$

and hence

$$
\sum_{A \in \mathscr{C}} \mathrm{m}\left(\tilde{\varphi}(t A) \Delta \sigma_{t} \tilde{\varphi}(A)\right) \leq \sum_{A \in \mathscr{C}} \mathrm{m}\left(\tilde{\varphi}(t A) \Delta \tilde{\varphi}^{\prime}(t A)\right)+\sum_{A \in \mathscr{C}} \mathrm{m}\left(\tilde{\varphi}^{\prime}(t A) \Delta \sigma_{t} \tilde{\varphi}^{\prime}(A)\right)
$$




$$
\begin{aligned}
&+\sum_{A \in \mathscr{C}} \mathrm{m}\left(\sigma_{t} \tilde{\varphi}^{\prime}(A) \Delta \sigma_{t} \tilde{\varphi}(A)\right) \\
& \stackrel{(13)}{\leq} \frac{\delta}{5}+\frac{\delta}{2}+\sum_{A \in \mathscr{C}} \mathrm{m}\left(\tilde{\varphi}^{\prime}(A) \Delta \tilde{\varphi}(A)\right) \\
& \stackrel{(12)}{\leq} \frac{\delta}{5}+\frac{\delta}{2}+\frac{\delta}{5}<\delta .
\end{aligned}
$$

Therefore the restriction of $\tilde{\varphi}$ to $\operatorname{alg}\left(\mathscr{C}_{L}\right)$ lies in $\operatorname{Hom}_{\mu}(\mathscr{C}, L, \delta, \sigma)$.

Lemma 4.8. Let $\mathscr{C}$ be a Borel finite partition of $X, L \in \overline{\mathcal{F}}(H)$, and $0<\delta<1$. Let $S \in \overline{\mathcal{F}}(G)$ and $L^{\bullet}, \bar{L} \in \overline{\mathcal{F}}(H)$ be such that $L^{\bullet} \subseteq \bar{L}$ and

$$
\mu\left(X \backslash X_{S, \bar{L}}\right) \leq \gamma:=\delta /\left(200|S| \cdot\left|L L^{\bullet}\right|\right) .
$$

Set $L^{\sharp}:=\bar{L} L \bar{L} \in \overline{\mathcal{F}}(H)$ and let $T \in \overline{\mathcal{F}}(G)$ be such that

$$
\mu\left(X \backslash X_{L^{\sharp}, T}\right) \leq \kappa:=\delta /\left(10^{4}|\bar{L}|^{2} \cdot\left|L L^{\bullet}\right|\right) .
$$

Let $C \in \mathbb{N}$ and $S_{1}, \ldots, S_{n} \in \overline{\mathcal{F}}(G)$, and let $W, \mathcal{V}_{1}, \ldots, \mathcal{V}_{n}$ be Borel subsets of $X$ such that $S W=X$, and for every $g \in T$ and $w \in W \cap g^{-1} W$ the points $w$ and $g w$ are connected by $a$ path of length at most $C$ in which each edge is an $S_{j}$-edge with both endpoints in $\mathcal{V}_{j}$ for some $1 \leq j \leq n$. Let $L^{\dagger} \in \overline{\mathcal{F}}(H)$ be such that

$$
\mu\left(X \backslash X_{\bigcup_{j=1}^{n} S_{j}, L^{\dagger}}\right) \leq \zeta:=\kappa /\left(100\left|\bigcup_{j=1}^{n} S_{j}\right|^{C}\right) .
$$

Let $0 \leq \bar{\tau} \leq \kappa /\left(10 C\left|\bigcup_{j=1}^{n} S_{j}\right|^{C} \cdot\left|L^{\dagger}\right|^{3 C}\right)$. Let $F \in \overline{\mathcal{F}}(G)$ be such that $T \cup\left(\bigcup_{j=1}^{n} S_{j}\right)^{C} \subseteq F$

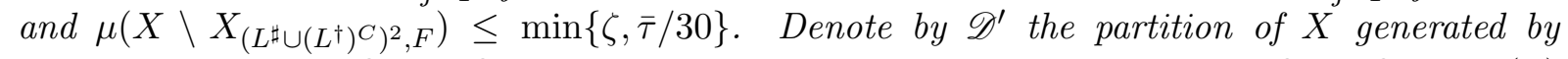
$W, \mathcal{V}_{1}, \ldots, \mathcal{V}_{n}$. Let $\mathscr{Q}_{1} \preceq \mathscr{Q}_{2}$ be finite Borel partitions of $W$ and let $\Theta: \mathscr{Q}_{1} \cup \mathscr{Q}_{2} \rightarrow \mathcal{F}(S)$ be such that

(i) $e_{G} \in \Theta\left(B_{2}\right)=\Theta\left(B_{1}\right)$ for all $B_{2} \in \mathscr{Q}_{2}$ and $B_{1} \in \mathscr{Q}_{1}$ with $B_{2} \subseteq B_{1}$,

(ii) for $i=1,2$ the sets $g B$ for $B \in \mathscr{Q}_{i}$ and $g \in \Theta(B)$ form a partition $\mathscr{R}_{i}$ of $X$,

(iii) $\mathscr{R}_{1} \succeq \mathscr{D}:=\mathscr{C}_{L} \vee\left(\mathscr{D}^{\prime}\right)_{T} \vee\left(\bigvee_{j=1}^{n} S_{j}, L^{\dagger} \mathscr{P}\right) \vee \bigcup_{j=1}^{n} S_{j}, L^{\dagger} \mathscr{P} \vee_{L^{\sharp}, T} \mathscr{P}$, and

(iv) $\mathscr{R}_{2} \succeq\left(\mathscr{R}_{1}\right)_{L \bar{L}}$.

Denote by $\mathscr{Q}_{1}^{\prime}$ the set of all $B \in \mathscr{Q}_{1}$ satisfying $B \subseteq X_{S, L} \bullet$, and denote by $\mathscr{Q}_{2}^{\prime}$ the set of all $B \in \mathscr{Q}_{2}$ satisfying $B \subseteq X_{S, \bar{L}}$. Denote by $\Lambda$ the set consisting of all $(B, g)$ for $B \in \mathscr{Q}_{2}^{\prime}$ and $g \in \Theta(B) \backslash\left\{e_{G}\right\}$. Let $0<\tau^{\prime} \leq \min \left\{\kappa /\left(100|F|^{3}\right), \bar{\tau} /\left(60|F|^{2}\right)\right\}$ and $0<\delta^{\prime} \leq \min \left\{\tau^{\prime}, \kappa /(10 n|F|), \delta /\left(50\left|L L^{\bullet}\right|(|S|+\right.\right.$ $1))\}$. Let $\mathscr{A}$ be a finite Borel partition of $X$ refining $\left(\mathscr{R}_{2}\right)_{\left(L^{\sharp} \cup\left(L^{\dagger}\right)^{C}\right)^{2}} \vee_{\left(L^{\sharp} \cup\left(L^{\dagger}\right)^{C}\right)^{2}, F} \mathscr{P} \vee \mathscr{D}_{\left(\bigcup_{j=1}^{n} S_{j}\right)^{C}}$. Let $\pi: G \rightarrow \operatorname{Sym}(V)$ be an $\left(F, \tau^{\prime}\right)$-approximation for $G$ and $\varphi, \varphi_{0} \in \operatorname{Hom}_{\mu}\left(\mathscr{A}, F, \delta^{\prime}, \pi\right)$. Let $\sigma: H \rightarrow \operatorname{Sym}(V)$ be an $\left(L^{\sharp} \cup\left(L^{\dagger}\right)^{C}, \bar{\tau}\right)$-approximation for $H$ such that $\rho_{\mathrm{Hamm}}\left(\sigma_{t}, \sigma_{t}^{\prime}\right) \leq \bar{\tau} / 5$ for all $t \in\left(L^{\sharp} \cup\left(L^{\dagger}\right)^{C}\right)^{2}$, where $\sigma_{t}^{\prime} \in V^{V}$ for $t \in\left(L^{\sharp} \cup\left(L^{\dagger}\right)^{C}\right)^{2}$ satisfies

$$
\sigma_{t}^{\prime} v=\pi_{\lambda(t, A)} v
$$

for all $A \in{ }_{\left(L^{\sharp} \cup\left(L^{\dagger}\right)^{C}\right)^{2}, F} \overline{\mathscr{P}}$ and $v \in \varphi_{0}(A)$. Define $\tilde{\varphi}^{\prime}: \mathscr{R}_{2} \rightarrow \mathbb{P}_{V}$ by $\tilde{\varphi}^{\prime}(B)=\varphi(B)$ for all $B \in \mathscr{Q}_{2}, \tilde{\varphi}^{\prime}(g B)=\emptyset$ for all $B \in \mathscr{Q}_{2} \backslash \mathscr{Q}_{2}^{\prime}$ and $g \in \Theta(B) \backslash\left\{e_{G}\right\}$, and

$$
\tilde{\varphi}^{\prime}(g B)=\sigma_{\kappa(g, B)} \varphi(B)
$$


for all $(B, g) \in \Lambda$, and extend $\tilde{\varphi}^{\prime}$ to a map $\operatorname{alg}\left(\mathscr{R}_{2}\right) \rightarrow \mathbb{P}_{V}$ by setting $\tilde{\varphi}^{\prime}(D)=\bigcup_{A \in \mathscr{R}_{2}, A \subseteq D} \tilde{\varphi}^{\prime}(A)$ for $D \in \operatorname{alg}\left(\mathscr{R}_{2}\right)$. Assume that $\varphi(W)=\varphi_{0}(W)$ and $\varphi\left(\mathcal{V}_{j} \cap D\right)=\varphi_{0}\left(\mathcal{V}_{j} \cap D\right)$ for all $1 \leq j \leq n$ and $D \in{ }_{S_{j}, L^{\dagger}} \mathscr{P}$. Then (7), (8), and (9) hold.

Proof. Denote by $V_{1}$ the set of all $v \in V$ satisfying $\pi_{g_{1} g_{2}} v \neq \pi_{g_{1}} \pi_{g_{2}} v$ for some $g_{1}, g_{2} \in\left(\bigcup_{j=1}^{n} S_{j}\right)^{C}$. Then $\mathrm{m}\left(V_{1}\right) \leq|F|^{2} \tau^{\prime} \leq \bar{\tau} / 60$. Denote by $V_{2}$ the set of all $v \in V$ satisfying $\sigma_{t}^{\prime} v \neq \sigma_{t} v$ for some $t \in L^{\dagger}$. Then $\mathrm{m}\left(V_{2}\right) \leq\left|L^{\dagger}\right| \bar{\tau} / 5$. Set $V_{3}=\bigcup_{g \in\left(\bigcup_{j=1}^{n} S_{j}\right)^{C}} \pi_{g}\left(V_{1} \cup V_{2}\right)$. Then

$$
\mathrm{m}\left(V_{3}\right) \leq\left|\bigcup_{j=1}^{n} S_{j}\right|^{C}\left(\mathrm{~m}\left(V_{1}\right)+\mathrm{m}\left(V_{2}\right)\right) \leq 2\left|\bigcup_{j=1}^{n} S_{j}\right|^{C} \cdot \frac{\left|L^{\dagger}\right| \bar{\tau}}{5} \leq \frac{\kappa}{25} .
$$

Denote by $V_{4}$ the set of all $v \in V$ satisfying $\sigma_{t_{1} t_{2}} v \neq \sigma_{t_{1}} \sigma_{t_{2}} v$ for some $t_{1}, t_{2} \in\left(L^{\dagger}\right)^{C}$. Then $\mathrm{m}\left(V_{4}\right) \leq\left|L^{\dagger}\right|^{2 C} \bar{\tau}$. Set $V_{5}=\bigcup_{l=1}^{C} \bigcup_{t_{1}, \ldots, t_{l} \in L^{\dagger}} \sigma_{t_{l}} \ldots \sigma_{t_{1}} V_{4}$. Then

$$
\mathrm{m}\left(V_{5}\right) \leq C\left|L^{\dagger}\right|^{C} \mathrm{~m}\left(V_{4}\right) \leq C\left|L^{\dagger}\right|^{3 C} \bar{\tau} \leq \frac{\kappa}{10} .
$$

Denote by $V_{6}$ the union of the sets $\varphi\left(g\left(\mathcal{V}_{j} \cap D\right)\right) \Delta \pi_{g} \varphi\left(\mathcal{V}_{j} \cap D\right)$ for $g \in\left(\bigcup_{i=1}^{n} S_{i}\right)^{C}, 1 \leq j \leq n$, and $D \in S_{S_{j}, L^{\dagger}} \mathscr{P}$. Then $\mathrm{m}\left(V_{6}\right) \leq n|F| \delta^{\prime} \leq \kappa / 10$. Also, denote by $V_{7}$ the union of the sets $\varphi(g A) \Delta \pi_{g} \varphi(A)$ for $A \in \mathscr{A}$ and $g \in T$. Then $\mathrm{m}\left(V_{7}\right) \leq|T| \delta^{\prime} \leq \kappa / 10$. Put

$$
V_{8}=\bigcup_{g \in\left(\bigcup_{j=1}^{n} S_{j}\right)^{C}} \pi_{g} \varphi_{0}\left(X \backslash\left(X_{\bigcup_{1 \leq j \leq n} S_{j}, L^{\dagger}} \cap X_{\left(L^{\sharp} \cup\left(L^{\dagger}\right)^{C}\right)^{2}, F}\right)\right) .
$$

Then $\mathrm{m}\left(V_{8}\right) \leq\left|\left(\bigcup_{j=1}^{n} S_{j}\right)^{C}\right|\left(2 \zeta+\delta^{\prime}\right) \leq \kappa / 5$. Set $V^{\prime}=V \backslash\left(V_{3} \cup V_{5} \cup V_{6} \cup V_{7} \cup V_{8}\right)$. We then have

$$
\mathrm{m}\left(V \backslash V^{\prime}\right) \leq \mathrm{m}\left(V_{3}\right)+\mathrm{m}\left(V_{5}\right)+\mathrm{m}\left(V_{6}\right)+\mathrm{m}\left(V_{7}\right)+\mathrm{m}\left(V_{8}\right) \leq \kappa .
$$

Denote by $\mathscr{Q}_{2}^{\prime \prime}$ the set of all $B \in \mathscr{Q}_{2}$ satisfying $B \subseteq X_{L^{\sharp}, T}$. Let $t \in L^{\sharp}$ and $B \in \mathscr{Q}_{2}^{\prime \prime}$. We claim that

$$
V^{\prime} \cap \varphi(W \cap t B) \subseteq \varphi(W) \cap \sigma_{t} \varphi(B) .
$$

Set $g=\lambda(t, B) \in T$. Denote by $\Xi_{t, B}$ the set consisting of all tuples $\xi=\left(k_{1}, \ldots, k_{l}, g_{1}, \ldots, g_{l}, D_{1}, \ldots, D_{l}\right)$ such that $1 \leq l \leq C, 1 \leq k_{1}, \ldots, k_{l} \leq n, g_{j} \in S_{k_{j}}$ for all $1 \leq j \leq l, g=g_{l} \ldots g_{1}$, and $D_{j} \in S_{k_{j}, L^{\dagger}} \mathscr{P}$ for all $1 \leq j \leq l$ and such that the set

$$
\Omega_{\xi}:=B \cap g^{-1} W \cap \bigcap_{j=1}^{l}\left(\left(g_{j} \cdots g_{1}\right)^{-1} \mathcal{V}_{k_{j}} \cap\left(g_{j-1} \cdots g_{1}\right)^{-1}\left(\mathcal{V}_{k_{j}} \cap D_{j}\right)\right) \in \operatorname{alg}(\mathscr{A})
$$

consisting of all $x \in B \cap t^{-1} W=B \cap g^{-1} W$ satisfying $g_{j} \ldots g_{1} x \in \mathcal{V}_{k_{j}}$ and $g_{j-1} \ldots g_{1} x \in \mathcal{V}_{k_{j}} \cap D_{j}$ for all $1 \leq j \leq l$ is nonempty. Then

$$
B \cap g^{-1} W=\bigcup_{\xi \in \Xi_{t, B}} \Omega_{\xi}
$$

Denote by $\Xi_{t, B}^{\prime}$ the set of all $\xi=\left(k_{1}, \ldots, k_{l}, g_{1}, \ldots, g_{l}, D_{1}, \ldots, D_{l}\right) \in \Xi_{t, B}$ such that $D_{j} \in S_{k_{j}, L^{\dagger}} \overline{\mathscr{P}}$ for all $1 \leq j \leq l$. For each $\xi=\left(k_{1}, \ldots, k_{l}, g_{1}, \ldots, g_{l}, D_{1}, \ldots, D_{l}\right) \in \Xi_{t, B}$, we have

$$
V^{\prime} \cap \varphi\left(g \Omega_{\xi}\right)
$$




$$
\begin{aligned}
& =V^{\prime} \cap \varphi\left(g B \cap W \cap \bigcap_{j=1}^{l}\left(\left(g_{l} \ldots g_{j+1} \mathcal{V}_{k_{j}}\right) \cap\left(g_{l} \ldots g_{j}\left(\mathcal{V}_{k_{j}} \cap D_{j}\right)\right)\right)\right) \\
& =V^{\prime} \cap \varphi(g B \cap W) \cap \bigcap_{j=1}^{l}\left(\varphi\left(g_{l} \ldots g_{j+1} \mathcal{V}_{k_{j}}\right) \cap \varphi\left(g_{l} \ldots g_{j}\left(\mathcal{V}_{k_{j}} \cap D_{j}\right)\right)\right) \\
& =V^{\prime} \cap \varphi(g B \cap W) \cap \bigcap_{j=1}^{l}\left(\pi_{g_{l} \ldots g_{j+1}} \varphi\left(\mathcal{V}_{k_{j}}\right) \cap \pi_{g_{l} \ldots g_{j}} \varphi\left(\mathcal{V}_{k_{j}} \cap D_{j}\right)\right) \\
& =V^{\prime} \cap \varphi(g B \cap W) \cap \bigcap_{j=1}^{l}\left(\pi_{g_{l} \ldots g_{j+1}} \varphi_{0}\left(\mathcal{V}_{k_{j}}\right) \cap \pi_{g_{l} \ldots g_{j}} \varphi_{0}\left(\mathcal{V}_{k_{j}} \cap D_{j}\right)\right) \\
& =V^{\prime} \cap \varphi(g B \cap W) \cap \bigcap_{j=1}^{l}\left(\pi_{g_{l} \ldots \pi_{g_{j+1}}} \varphi_{0}\left(\mathcal{V}_{k_{j}}\right) \cap \pi_{g_{l}} \ldots \pi_{g_{j+1}} \pi_{g_{j}} \varphi_{0}\left(\mathcal{V}_{k_{j}} \cap D_{j}\right)\right) \\
& =V^{\prime} \cap \varphi(g B \cap W) \cap \bigcap_{j=1}^{l} \pi_{g_{l}} \ldots \pi_{g_{j+1}}\left(\varphi_{0}\left(\mathcal{V}_{k_{j}}\right) \cap \pi_{g_{j}} \varphi_{0}\left(\mathcal{V}_{k_{j}} \cap D_{j}\right)\right) .
\end{aligned}
$$

If $\xi \in \Xi_{t, B} \backslash \Xi_{t, B}^{\prime}$, then $D_{j}=X \backslash X_{S_{k_{j}} L^{\dagger}} \subseteq X \backslash X_{\bigcup_{i=1}^{n} S_{i}, L^{\dagger}}$ for some $1 \leq j \leq l$ and hence $V^{\prime} \cap \varphi\left(g \Omega_{\xi}\right)=\emptyset$. Thus

$$
\begin{aligned}
V^{\prime} \cap \varphi(W \cap t B)=V^{\prime} \cap \varphi & (g B \cap W)=V^{\prime} \cap \varphi\left(\bigcup_{\xi \in \Xi_{t, B}} g \Omega_{\xi}\right) \\
= & \bigcup_{\xi \in \Xi_{t, B}}\left(V^{\prime} \cap \varphi\left(g \Omega_{\xi}\right)\right)=\bigcup_{\xi \in \Xi_{t, B}^{\prime}}\left(V^{\prime} \cap \varphi\left(g \Omega_{\xi}\right)\right) .
\end{aligned}
$$

Now let $\xi \in \Xi_{t, B}^{\prime}$. Let $w \in V^{\prime} \cap \varphi\left(g \Omega_{\xi}\right)$. For each $1 \leq j \leq l$ one has $w=\pi_{g_{l} \ldots g_{j+1}} w_{j}=$ $\pi_{g_{l}} \ldots \pi_{g_{j+1}} w_{j}$ for some $w_{j} \in \varphi_{0}\left(\mathcal{V}_{k_{j}}\right) \cap \pi_{g_{j}} \varphi_{0}\left(\mathcal{V}_{k_{j}} \cap D_{j}\right)$. We can also find some $w_{0} \in V$ such that $w=\pi_{g_{l}} \ldots \pi_{g_{1}} w_{0}=\pi_{g} w_{0}$. Note that

$$
w_{0}=\pi_{g}^{-1} w \in \pi_{g}^{-1}\left(V^{\prime} \cap \varphi\left(g \Omega_{\xi}\right)\right) \subseteq \varphi\left(\Omega_{\xi}\right) \subseteq \varphi(B) .
$$

We have $w_{j}=\pi_{g_{j}} w_{j-1}$ for all $1 \leq j \leq l$, and hence $w_{j-1} \in \varphi_{0}\left(\mathcal{V}_{k_{j}} \cap D_{j}\right) \cap \varphi_{0}\left(X_{\left(L^{\sharp}\left(L^{\dagger}\right)^{C}\right)^{2}, F}\right)$ for all $1 \leq j \leq l$. Set $t_{j}=\kappa\left(g_{j}, D_{j}\right) \in L^{\dagger}$ for $1 \leq j \leq l$. Then $w_{j}=\pi_{g_{j}} w_{j-1}=\sigma_{t_{j}}^{\prime} w_{j-1}=\sigma_{t_{j}} w_{j-1}$ for all $1 \leq j \leq l$. Therefore $w=\sigma_{t_{l}} \ldots \sigma_{t_{1}} w_{0}=\sigma_{t_{l} \ldots t_{1}} w_{0}$. Take $x \in \Omega_{\xi}$. We have

$$
\begin{aligned}
t=\kappa(g, x)=\kappa\left(g_{l} \ldots g_{1}, x\right) & =\kappa\left(g_{l} \ldots g_{2}, g_{1} x\right) \kappa\left(g_{1}, x\right) \\
& =\kappa\left(g_{l} \ldots g_{2}, g_{1} x\right) t_{1}=\cdots=t_{l} \ldots t_{1} .
\end{aligned}
$$

Thus $w=\sigma_{t} w_{0} \in \sigma_{t} \varphi(B)$ and hence $V^{\prime} \cap \varphi\left(g \Omega_{\xi}\right) \subseteq \varphi(W) \cap \sigma_{t} \varphi(B)$. Therefore

$$
V^{\prime} \cap \varphi(W \cap t B)=\bigcup_{\xi \in \Xi_{t, B}^{\prime}}\left(V^{\prime} \cap \varphi\left(g \Omega_{\xi}\right)\right) \subseteq \varphi(W) \cap \sigma_{t} \varphi(B) .
$$

This proves our claim (16). 
Now let $t \in L^{\sharp}$. Applying Lemma 4.3 with $\mathscr{C}=\mathscr{R}_{2}, L=L^{\sharp} \cup\left(L^{\dagger}\right)^{C}, \tau=\bar{\tau}, F=F, \tau^{\prime}=\tau^{\prime}, \pi=$ $\pi, \sigma=\sigma, \sigma^{\prime}=\sigma^{\prime}$, and $\varphi$ being the restriction of $\varphi_{0}$ to $\operatorname{alg}\left(\left(\left(\mathscr{R}_{2}\right)_{\left(L^{\sharp} \cup\left(L^{\dagger}\right)^{C}\right)^{2}} \vee_{\left(L^{\sharp} \cup\left(L^{\dagger}\right)^{C}\right)^{2}, F} \mathscr{P}\right)_{F}\right)$, we have that the restriction of $\varphi_{0}$ to $\operatorname{alg}\left(\left(\mathscr{R}_{2}\right)_{L^{\sharp} \cup\left(L^{\dagger}\right)^{C}}\right)$ lies in $\operatorname{Hom}_{\mu}\left(\mathscr{R}_{2}, L^{\sharp} \cup\left(L^{\dagger}\right)^{C}, \bar{\tau}, \sigma\right)$. Thus

$$
\mathrm{m}\left(\left(\varphi_{0}(W) \cap \sigma_{t} \varphi_{0}(W)\right) \Delta\left(\varphi_{0}(W) \cap \varphi_{0}(t W)\right) \leq \mathrm{m}\left(\sigma_{t} \varphi_{0}(W) \Delta \varphi_{0}(t W)\right) \leq \bar{\tau} .\right.
$$

Therefore

$$
\begin{aligned}
\mathrm{m}\left(\varphi_{0}(W) \cap \sigma_{t} \varphi_{0}(W)\right) & \leq \mathrm{m}\left(\varphi_{0}(W) \cap \varphi_{0}(t W)\right)+\bar{\tau} \\
& =\mathrm{m}\left(\varphi_{0}(W \cap t W)\right)+\bar{\tau} \\
& \leq \mu(W \cap t W)+\delta^{\prime}+\bar{\tau} \\
& \leq \mathrm{m}(\varphi(W \cap t W))+2 \delta^{\prime}+\bar{\tau} \\
& \leq \mathrm{m}\left(V^{\prime} \cap \varphi(W \cap t W)\right)+\kappa+2 \delta^{\prime}+\bar{\tau} .
\end{aligned}
$$

Also note that

$$
\begin{aligned}
& \mathrm{m}\left(\left(V^{\prime} \cap \varphi(W \cap t W)\right) \backslash\left(V^{\prime} \cap \varphi\left(W \cap t\left(W \cap X_{L^{\sharp}, T}\right)\right)\right)\right) \\
& \leq \mathrm{m}\left(\varphi(W \cap t W) \backslash \varphi\left(W \cap t\left(W \cap X_{L^{\sharp}, T}\right)\right)\right) \\
&=\mathrm{m}\left(\varphi\left((W \cap t W) \backslash\left(W \cap t\left(W \cap X_{L^{\sharp}, T}\right)\right)\right)\right) \\
& \leq \mathrm{m}\left(\varphi\left(t\left(X \backslash X_{L^{\sharp}, T}\right)\right)\right) \\
& \leq \delta^{\prime}+\mu\left(t\left(X \backslash X_{L^{\sharp}, T}\right)\right) \leq \delta^{\prime}+\kappa,
\end{aligned}
$$

and hence

$$
\mathrm{m}\left(V^{\prime} \cap \varphi(W \cap t W)\right) \leq \mathrm{m}\left(V^{\prime} \cap \varphi\left(W \cap t\left(W \cap X_{L^{\sharp}, T}\right)\right)\right)+\delta^{\prime}+\kappa .
$$

Then

$$
\begin{aligned}
\sum_{B \in \mathscr{Q}_{2}} \mathrm{~m} & \left(\left(\varphi(W) \cap \sigma_{t} \varphi(B)\right) \backslash \varphi(W \cap t B)\right) \\
& \leq \sum_{B \in \mathscr{Q}_{2} \backslash \mathscr{Q}_{2}^{\prime \prime}} \mathrm{m}\left(\varphi(W) \cap \sigma_{t} \varphi(B)\right)+\sum_{B \in \mathscr{Q}_{2}^{\prime \prime}} \mathrm{m}\left(\left(\varphi(W) \cap \sigma_{t} \varphi(B)\right) \backslash \varphi(W \cap t B)\right) \\
& \leq \sum_{B \in \mathscr{Q}_{2} \backslash \mathscr{Q}_{2}^{\prime \prime}} \mathrm{m}(\varphi(B))+\sum_{B \in \mathscr{Q}_{2}^{\prime \prime}} \mathrm{m}\left(\left(\varphi(W) \cap \sigma_{t} \varphi(B)\right) \backslash\left(V^{\prime} \cap \varphi(W \cap t B)\right)\right) \\
& \stackrel{(16)}{=} \mathrm{m}\left(\varphi\left(W \backslash X_{L^{\sharp}, T}\right)\right) \\
& \quad+\mathrm{m}\left(\left(\varphi(W) \cap \sigma_{t} \varphi\left(W \cap X_{L^{\sharp}, T}\right)\right) \backslash\left(V^{\prime} \cap \varphi\left(W \cap t\left(W \cap X_{L^{\sharp}, T}\right)\right)\right)\right) \\
& \leq \mathrm{m}\left(\varphi\left(X \backslash X_{L^{\sharp}, T}\right)\right)+\mathrm{m}\left(\varphi(W) \cap \sigma_{t} \varphi(W)\right)-\mathrm{m}\left(V^{\prime} \cap \varphi\left(W \cap t\left(W \cap X_{L^{\sharp}, T}\right)\right)\right) \\
& \stackrel{(18)}{\leq} \mu\left(X \backslash X_{L^{\sharp}, T}\right)+\delta^{\prime}+\mathrm{m}\left(\varphi_{0}(W) \cap \sigma_{t} \varphi_{0}(W)\right) \\
& \quad-\mathrm{m}\left(V^{\prime} \cap \varphi(W \cap t W)\right)+\delta^{\prime}+\kappa \\
& \quad \leq \kappa+\delta^{\prime}+\kappa+2 \delta^{\prime}+\bar{\tau}+\delta^{\prime}+\kappa=3 \kappa+4 \delta^{\prime}+\bar{\tau} \leq 8 \kappa,
\end{aligned}
$$


and

$$
\begin{aligned}
& \sum_{B \in \mathscr{Q}_{2}} \mathrm{~m}\left(\varphi(W \cap t B) \backslash\left(\varphi(W) \cap \sigma_{t} \varphi(B)\right)\right) \\
& \quad \leq \sum_{B \in \mathscr{Q}_{2} \backslash \mathscr{Q}_{2}^{\prime \prime}} \mathrm{m}(\varphi(W \cap t B))+\sum_{B \in \mathscr{Q}_{2}^{\prime \prime}} \mathrm{m}\left(\varphi(W \cap t B) \backslash\left(\varphi(W) \cap \sigma_{t} \varphi(B)\right)\right) \\
& \quad \stackrel{\text { (16) }}{\leq} \mathrm{m}\left(\varphi\left(W \cap t\left(W \backslash X_{L^{\sharp}, T}\right)\right)\right)+\mathrm{m}\left(V \backslash V^{\prime}\right) \\
& \quad \leq \mathrm{m}\left(\varphi\left(t\left(X \backslash X_{L^{\sharp}, T}\right)\right)+\kappa\right. \\
& \quad \leq \mu\left(t\left(X \backslash X_{L^{\sharp}, T}\right)\right)+\delta^{\prime}+\kappa \leq 2 \kappa+\delta^{\prime} .
\end{aligned}
$$

For each $(B, g) \in \Lambda$, we have $\tilde{\varphi}^{\prime}(g B)=\sigma_{\kappa(g, B)} \varphi(B), \kappa(g, B) \in \bar{L}$, and $W \cap \kappa(g, B) B=$ $W \cap g B=\emptyset$. Thus

$$
\begin{aligned}
\sum_{(B, g) \in \Lambda} \mathrm{m}\left(\tilde{\varphi}^{\prime}(g B) \cap \varphi(W)\right) & =\sum_{(B, g) \in \Lambda} \mathrm{m}\left(\sigma_{\kappa(g, B)} \varphi(B) \cap \varphi(W)\right) \\
& \leq \sum_{t \in \bar{L}} \sum_{\substack{B \in \mathscr{Q}_{2}^{\prime}, t B \cap W=\emptyset}} \mathrm{m}\left(\sigma_{t} \varphi(B) \cap \varphi(W)\right) \\
& \stackrel{(19))}{\leq}|\bar{L}| 8 \kappa \\
& \leq \frac{\delta}{40}
\end{aligned}
$$

verifying (17).

For distinct $\left(B_{1}, g_{1}\right),\left(B_{2}, g_{2}\right)$ in $\Lambda$, we have $\kappa\left(g_{1}, B_{1}\right) B_{1} \cap \kappa\left(g_{2}, B_{2}\right) B_{2}=\emptyset$, and hence $B_{1} \cap$ $\kappa\left(g_{1}, B_{1}\right)^{-1} \kappa\left(g_{2}, B_{2}\right) B_{2}=\emptyset$. For any $t_{1}, t_{2} \in L \bar{L}$, we have

$$
\begin{aligned}
\rho_{\text {Hamm }}\left(\sigma_{t_{1}}^{-1} \sigma_{t_{2}}, \sigma_{t_{1}^{-1} t_{2}}\right) & \leq \rho_{\text {Hamm }}\left(\sigma_{t_{1}}^{-1}, \sigma_{t_{1}^{-1}}\right)+\rho_{\text {Hamm }}\left(\sigma_{t_{1}^{-1}} \sigma_{t_{2}}, \sigma_{t_{1}^{-1} t_{2}}\right) \\
& \leq \rho_{\text {Hamm }}\left(\operatorname{id}_{V}, \sigma_{t_{1}} \sigma_{t_{1}^{-1}}\right)+\bar{\tau} \\
& \leq \rho_{\text {Hamm }}\left(\operatorname{id}_{V}, \sigma_{e_{H}}\right)+\rho_{\text {Hamm }}\left(\sigma_{e_{H}}, \sigma_{t_{1}} \sigma_{t_{1}^{-1}}\right)+\bar{\tau} \\
& =\rho_{\text {Hamm }}\left(\sigma_{e_{H}}, \sigma_{e_{H}} \sigma_{e_{H}}\right)+\rho_{\text {Hamm }}\left(\sigma_{e_{H}}, \sigma_{t_{1}} \sigma_{t_{1}^{-1}}\right)+\bar{\tau} \\
& \leq 3 \bar{\tau} .
\end{aligned}
$$

Thus

$$
\begin{aligned}
& \sum_{\substack{\left(B_{1}, g_{1}\right),\left(B_{2}, g_{2}\right) \in \Lambda,\left(B_{1}, g_{1}\right) \neq\left(B_{2}, g_{2}\right)}} \mathrm{m}\left(\tilde{\varphi}^{\prime}\left(g_{1} B_{1}\right) \cap \tilde{\varphi}^{\prime}\left(g_{2} B_{2}\right)\right) \\
& =\sum_{\substack{\left(B_{1}, g_{1}\right),\left(B_{2}, g_{2}\right) \in \Lambda,\left(B_{1}, g_{1}\right) \neq\left(B_{2}, g_{2}\right)}} \mathrm{m}\left(\sigma_{\kappa\left(g_{1}, B_{1}\right)} \varphi\left(B_{1}\right) \cap \sigma_{\kappa\left(g_{2}, B_{2}\right)} \varphi\left(B_{2}\right)\right) \\
& \leq \sum_{t_{1}, t_{2} \in \bar{L}} \sum_{\substack{B_{1}, B_{2} \in \mathscr{Q}_{2}^{\prime}, B_{1} \cap t_{1}^{-1} t_{2} B_{2}=\emptyset}} \mathrm{m}\left(\sigma_{t_{1}} \varphi\left(B_{1}\right) \cap \sigma_{t_{2}} \varphi\left(B_{2}\right)\right)
\end{aligned}
$$




$$
\begin{aligned}
& =\sum_{t_{1}, t_{2} \in \bar{L}} \sum_{\substack{B_{1}, B_{2} \in \mathscr{Q}_{2}^{\prime}, B_{1} \cap t_{1}^{-1} t_{2} B_{2}=\emptyset}} \mathrm{m}\left(\varphi\left(B_{1}\right) \cap \sigma_{t_{1}}^{-1} \sigma_{t_{2}} \varphi\left(B_{2}\right)\right) \\
& \leq \sum_{t_{1}, t_{2} \in \bar{L}}\left[\rho_{\operatorname{Hamm}}\left(\sigma_{t_{1}}^{-1} \sigma_{t_{2}}, \sigma_{t_{1}^{-1} t_{2}}\right)+\sum_{\substack{B_{1}, B_{2} \in \mathscr{Q}_{2}^{\prime}, B_{1} \cap t_{1}^{-1} t_{2} B_{2}=\emptyset}} \mathrm{m}\left(\varphi\left(B_{1}\right) \cap \sigma_{t_{1}^{-1} t_{2}} \varphi\left(B_{2}\right)\right)\right] \\
& \stackrel{(21))}{\leq} 3|\bar{L}|^{2} \bar{\tau}+\sum_{t_{1}, t_{2} \in \bar{L}} \sum_{\substack{B_{1}, B_{2} \in \mathscr{Q}_{2}^{\prime}, B_{1} \cap t_{1}^{-1} t_{2} B_{2}=\emptyset}} \mathrm{m}\left(\varphi\left(B_{1}\right) \cap \varphi(W) \cap \sigma_{t_{1}^{-1} t_{2}} \varphi\left(B_{2}\right)\right) \\
& \stackrel{(19)}{\leq} 3|\bar{L}|^{2} \bar{\tau}+|\bar{L}|^{2} 8 \kappa+\sum_{t_{1}, t_{2} \in \bar{L}} \sum_{\substack{B_{1}, B_{2} \in \mathscr{Q}_{2}^{\prime}, B_{1} \cap t_{1}^{-1} t_{2} B_{2}=\emptyset}} \mathrm{m}\left(\varphi\left(B_{1}\right) \cap \varphi\left(W \cap t_{1}^{-1} t_{2} B_{2}\right)\right) \\
& =|\bar{L}|^{2}(3 \bar{\tau}+8 \kappa) \leq \frac{\delta}{40},
\end{aligned}
$$

verifying (8).

For each $t \in L L^{\bullet}$, we have

$$
\begin{aligned}
& \sum_{B \in \mathscr{Q}_{1}^{\prime}} \mathrm{m}\left(\sigma_{t} \varphi(B) \backslash \tilde{\varphi}^{\prime}(t B)\right) \\
& =\sum_{B \in \mathscr{Q}_{1}^{\prime}} \mathrm{m}\left(\varphi(B) \backslash \sigma_{t}^{-1} \tilde{\varphi}^{\prime}(t B)\right) \\
& =\sum_{B \in \mathscr{Q}_{1}^{\prime}}\left[\mathrm{m}(\varphi(B))-\mathrm{m}\left(\varphi(B) \cap \sigma_{t}^{-1} \tilde{\varphi}^{\prime}(t B)\right)\right]
\end{aligned}
$$

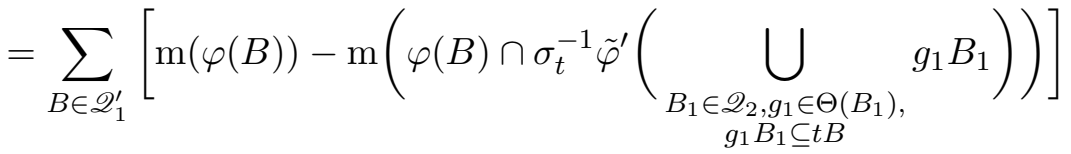

$$
\begin{aligned}
& =\sum_{B \in \mathscr{Q}_{1}^{\prime}}\left[\mathrm{m}(\varphi(B))-\mathrm{m}\left(\bigcup_{\substack{B_{1} \in \mathscr{Q}_{2}, g_{1} \in \Theta\left(B_{1}\right), g_{1} B_{1} \subseteq t B}}\left(\varphi(B) \cap \sigma_{t}^{-1} \tilde{\varphi}^{\prime}\left(g_{1} B_{1}\right)\right)\right)\right] \\
& =\sum_{B \in \mathscr{Q}_{1}^{\prime}}\left[\mathrm{m}(\varphi(B))-\mathrm{m}\left(\bigcup_{B_{1} \in \mathscr{Q}_{2}, B_{1} \subseteq t B}\left(\varphi(B) \cap \sigma_{t}^{-1} \varphi\left(B_{1}\right)\right)\right)\right. \\
& \left.-\mathrm{m}\left(\bigcup_{\substack{\left(B_{1}, g_{1}\right) \in \Lambda, g_{1} B_{1} \subseteq t B}}\left(\varphi(B) \cap \sigma_{t}^{-1} \sigma_{\kappa\left(g_{1}, B_{1}\right)} \varphi\left(B_{1}\right)\right)\right)\right] \\
& \leq \sum_{B \in \mathscr{Q}_{1}^{\prime}}\left[\mathrm{m}(\varphi(B))-\mathrm{m}\left(\bigcup_{B_{1} \in \mathscr{Q}_{2}^{\prime}, B_{1} \subseteq t B}\left(\varphi(B) \cap \sigma_{t}^{-1} \sigma_{e_{H}} \varphi\left(B_{1}\right)\right)\right)\right.
\end{aligned}
$$




$$
\begin{aligned}
& \left.-\mathrm{m}\left(\bigcup_{\substack{\left(B_{1}, g_{1}\right) \in \Lambda, g_{1} B_{1} \subseteq t B}}\left(\varphi(B) \cap \sigma_{t}^{-1} \sigma_{\kappa\left(g_{1}, B_{1}\right)} \varphi\left(B_{1}\right)\right)\right)\right]+\rho_{\mathrm{Hamm}}\left(\sigma_{e_{H}}, \operatorname{id}_{V}\right) \\
& =\sum_{B \in \mathscr{Q}_{1}^{\prime}}\left[\mathrm{m}(\varphi(B))-\mathrm{m}\left(\bigcup_{\substack { t_{1} \in \bar{L} \\
\begin{subarray}{c}{\lambda\left(t_{1}, B_{1}\right) \in \Theta\left(B_{1}\right), t_{1} B_{1} \subseteq t B{ t _ { 1 } \in \overline { L } \\
\begin{subarray} { c } { \lambda ( t _ { 1 } , B _ { 1 } ) \in \Theta ( B _ { 1 } ) , \\
t _ { 1 } B _ { 1 } \subseteq t B } }\end{subarray}}\left(\varphi(B) \cap \varphi(W) \cap \sigma_{t}^{-1} \sigma_{t_{1}} \varphi\left(B_{1}\right)\right)\right)\right] \\
& +\rho_{\text {Hamm }}\left(\sigma_{e_{H}} \sigma_{e_{H}}, \sigma_{e_{H}}\right) \\
& \leq \sum_{B \in \mathscr{Q}_{1}^{\prime}}\left[\mathrm{m}(\varphi(B))-\mathrm{m}\left(\bigcup_{t_{1} \in \bar{L}} \bigcup_{\substack{B_{1} \in \mathscr{Q}_{2}^{\prime}, \lambda\left(t_{1}, B_{1}\right) \in \Theta\left(B_{1}\right), t_{1} B_{1} \subseteq t B}}\left(\varphi(B) \cap \varphi(W) \cap \sigma_{t^{-1} t_{1}} \varphi\left(B_{1}\right)\right)\right)\right] \\
& +\sum_{t_{1} \in \bar{L}} \rho_{\mathrm{Hamm}}\left(\sigma_{t}^{-1} \sigma_{t_{1}}, \sigma_{t^{-1} t_{1}}\right)+\bar{\tau} \\
& \stackrel{\text { (20), (21) }}{\leq} \sum_{B \in \mathscr{Q}_{1}^{\prime}}\left[\mathrm{m}(\varphi(B))-\mathrm{m}\left(\bigcup_{t_{1} \in \bar{L}} \bigcup_{\substack{B_{1} \in \mathscr{Q}_{2}^{\prime}, \lambda\left(t_{1}, B_{1}\right) \in \Theta\left(B_{1}\right), t_{1} B_{1} \subseteq t B}}\left(\varphi(B) \cap \varphi\left(W \cap t^{-1} t_{1} B_{1}\right)\right)\right)\right] \\
& +|\bar{L}|\left(2 \kappa+\delta^{\prime}\right)+3|\bar{L}| \bar{\tau}+\bar{\tau} \\
& \leq \sum_{B \in \mathscr{Q}_{1}^{\prime}}\left[\mathrm{m}(\varphi(B))-\mathrm{m}\left(\bigcup_{\substack{t_{1} \in \bar{L}, B_{1} \in \mathscr{Q}_{2}^{\prime}, \lambda\left(t_{1}, B_{1}\right) \in \Theta\left(B_{1}\right), t_{1} B_{1} \subseteq t B}} \varphi\left(B \cap t^{-1} t_{1} B_{1}\right)\right)\right]+|\bar{L}|\left(2 \kappa+\delta^{\prime}+4 \bar{\tau}\right) \\
& =\sum_{B \in \mathscr{Q}_{1}^{\prime}} \mathrm{m}\left(\varphi\left(B \cap \bigcup_{\substack{B_{1} \in \mathscr{Q}_{2} \backslash \mathscr{Q}_{2}^{\prime}, g_{1} \in \Theta\left(B_{1}\right)}} t^{-1} g_{1} B_{1}\right)\right)+|\bar{L}|\left(2 \kappa+\delta^{\prime}+4 \bar{\tau}\right) \\
& \leq \mathrm{m}\left(\varphi\left(\bigcup_{\substack{B_{1} \in \mathscr{Q}_{2} \backslash \mathscr{Q}_{2}^{\prime}, g_{1} \in \Theta\left(B_{1}\right)}} t^{-1} g_{1} B_{1}\right)\right)+|\bar{L}|\left(2 \kappa+\delta^{\prime}+4 \bar{\tau}\right) \\
& \leq \mu\left(\bigcup_{\substack{B_{1} \in \mathscr{Q}_{2} \backslash \mathscr{Q}_{2}^{\prime}, g_{1} \in \Theta\left(B_{1}\right)}} t^{-1} g_{1} B_{1}\right)+\delta^{\prime}+|\bar{L}|\left(2 \kappa+\delta^{\prime}+4 \bar{\tau}\right) \\
& \leq|S| \mu\left(X \backslash X_{S, \bar{L}}\right)+|\bar{L}|\left(2 \kappa+2 \delta^{\prime}+4 \bar{\tau}\right) \\
& \leq|S| \gamma+|\bar{L}| 8 \kappa \leq \frac{\delta}{100\left|L L^{\bullet}\right|},
\end{aligned}
$$

and also

$$
\sum_{B \in \mathscr{Q}_{1}^{\prime}} \mathrm{m}\left(\tilde{\varphi}^{\prime}(t B)\right) \leq \sum_{B \in \mathscr{Q}_{1}^{\prime}} \sum_{\substack{B_{1} \in \mathscr{Q}_{2}, g_{1} \in \Theta\left(B_{1}\right), g_{1} B_{1} \subseteq t B}} \mathrm{~m}\left(\tilde{\varphi}^{\prime}\left(g_{1} B_{1}\right)\right)
$$




$$
\begin{aligned}
& \leq \sum_{B \in \mathscr{Q}_{1}^{\prime}} \sum_{\substack{B_{1} \in \mathscr{Q}_{2}, g_{1} \in \Theta\left(B_{1}\right), g_{1} B_{1} \subseteq t B}} \mathrm{~m}\left(\varphi\left(B_{1}\right)\right) \\
& \leq|S| \delta^{\prime}+\sum_{B \in \mathscr{Q}_{1}^{\prime}} \sum_{\substack{B_{1} \in \mathscr{Q}_{2}, g_{1} \in \Theta\left(B_{1}\right), g_{1} B_{1} \subseteq t B}} \mu\left(B_{1}\right) \\
& =|S| \delta^{\prime}+\sum_{B \in \mathscr{Q}_{1}^{\prime}} \sum_{\substack{B_{1} \in \mathscr{Q}_{2}, g_{1} \in \Theta\left(B_{1}\right), g_{1} B_{1} \subseteq t B}} \mu\left(g_{1} B_{1}\right) \\
& =|S| \delta^{\prime}+\sum_{B \in \mathscr{Q}_{1}^{\prime}} \mu(B) \\
& \leq(|S|+1) \delta^{\prime}+\sum_{B \in \mathscr{Q}_{1}^{\prime}} \mathrm{m}(\varphi(B)) .
\end{aligned}
$$

Thus

$$
\begin{array}{rl}
\sum_{B \in \mathscr{Q}_{1}^{\prime}} \sum_{t \in L L} & \mathrm{~m}\left(\sigma_{t} \varphi(B) \Delta \tilde{\varphi}^{\prime}(t B)\right) \\
& =\sum_{B \in \mathscr{Q}_{1}^{\prime}} \sum_{t \in L L^{\bullet}}\left[2 \mathrm{~m}\left(\sigma_{t} \varphi(B) \backslash \tilde{\varphi}^{\prime}(t B)\right)+\mathrm{m}\left(\tilde{\varphi}^{\prime}(t B)\right)-\mathrm{m}\left(\sigma_{t} \varphi(B)\right)\right] \\
& \stackrel{(22)}{\leq} \frac{\delta}{50}+\sum_{B \in \mathscr{Q}_{1}^{\prime}} \sum_{t \in L L^{\bullet}}\left[\mathrm{m}\left(\tilde{\varphi}^{\prime}(t B)\right)-\mathrm{m}(\varphi(B))\right] \\
& \stackrel{(23)}{\leq} \frac{\delta}{50}+\left|L L^{\bullet}\right|(|S|+1) \delta^{\prime} \leq \frac{\delta}{20}
\end{array}
$$

verifying (91).

For $F \in \mathcal{F}(G)$ and $L \in \mathcal{F}(H)$ we denote by ${ }_{F, L} \mathscr{W}$ the countable Borel partition of $X$ consisting of $X_{F, L}$ and $P \in{ }_{F} \mathscr{P} \backslash_{F, L} \mathscr{P}$. For any $F \in \mathcal{F}(G)$, the fact that ${ }_{F} \mathscr{P}$ has finite Shannon entropy means that for every $\varepsilon>0$ we can find a $\Gamma(F, \varepsilon) \in \mathcal{F}(H)$ such that $H_{\mu}\left({ }_{F, \Gamma(F, \varepsilon)} \mathscr{W}\right)<\varepsilon$.

Proof of Theorem 4.1. We may assume that $h_{\mu}(G \curvearrowright X) \neq-\infty$, which means in particular that $G$ is sofic.

By Stirling's formula there is a function $\Psi:(0,1) \rightarrow(0,1)$ such that for any nonempty finite set $V$ and any $0<\varepsilon<1$ the number of subsets $V^{\prime}$ of $V$ satisfying $\left|V^{\prime}\right| /|V| \leq \Psi(\varepsilon)$ is at most $e^{\varepsilon|V|}$.

Let $\Pi=\left\{\pi_{k}: G \rightarrow \operatorname{Sym}\left(V_{k}\right)\right\}_{k=1}^{\infty}$ be a sofic approximation sequence for $G$ and $\overline{\mathscr{C}}$ a finite Borel partition of $X$ with $h_{\Pi, \mu}(G \curvearrowright X, \overline{\mathscr{C}}) \geq 0$. Let $0<\varepsilon<1$. To establish the theorem it is enough to show the existence of a sofic approximation sequence $\Sigma$ for $H$ and a finite Borel partition $\mathscr{C}^{b}$ of $X$ such that $h_{\Sigma, \mu}\left(H \curvearrowright X, \mathscr{C}^{b}\right) \geq h_{\Pi, \mu}(G \curvearrowright X, \overline{\mathscr{C}})-6 \varepsilon$.

Enumerate the elements of $\mathcal{F}(G)$ as $\bar{F}_{1}, \bar{F}_{2}, \ldots$ Take two decreasing sequences $1>\delta_{1}>$ $\delta_{2}>\ldots$ and $1>\tau_{1}>\tau_{2}>\ldots$ converging to 0 , an increasing sequence $\left\{L_{k}\right\}_{k \in \mathbb{N}}$ in $\overline{\mathcal{F}}(H)$ with union $H$, and an increasing sequence $\left\{\mathscr{U}_{k}\right\}_{k \in \mathbb{N}}$ of finite Borel partitions of $X$ such that the algebra generated by $\bigcup_{k \in \mathbb{N}} \mathscr{U}_{k}$ is dense in the Borel $\sigma$-algebra of $X$ with respect to the 
pseudometric $d(A, B)=\mu(A \Delta B)$ (such a sequence can be found in view of the fact that every atomless standard probability space is measure isomorphic to the unit interval equipped with the Lebesgue measure on its Borel $\sigma$-algebra [24, Theorem A.20]).

We define $\Upsilon: \mathcal{F}(G) \rightarrow[0, \infty)$ by $\Upsilon\left(\bar{F}_{\ell}\right)=2 /\left(\Psi(\varepsilon) \Psi\left(\varepsilon /\left(\left.2^{\ell}\right|_{\bar{F}_{\ell}, \Gamma\left(\bar{F}_{\ell}, \varepsilon / 2^{\ell}\right)} \mathscr{P} \mid\right)\right)\right)$ for all $\ell \in \mathbb{N}$.

Since $G \curvearrowright(X, \mu)$ has property SC, there is an $S \in \overline{\mathcal{F}}(G)$ such that for any $T_{k} \in \overline{\mathcal{F}}(G)$ there are $n_{k}, C_{k} \in \mathbb{N}, S_{k, 1}, \ldots, S_{k, n_{k}} \in \overline{\mathcal{F}}(G)$, and Borel sets $W_{k}, \mathcal{V}_{k, 1}, \ldots, \mathcal{V}_{k, n_{k}} \subseteq X$ satisfying the following conditions:

(i) $\sum_{j=1}^{n_{k}} \Upsilon\left(S_{k, j}\right) \mu\left(\mathcal{V}_{k, j}\right) \leq 1$,

(ii) $S W_{k}=X$,

(iii) if $w_{1}, w_{2} \in W_{k}$ satisfy $g w_{1}=w_{2}$ for some $g \in T_{k}$ then $w_{1}$ and $w_{2}$ are connected by a path of length at most $C_{k}$ in which each edge is an $S_{k, j}$-edge with both endpoints in $\mathcal{V}_{k, j}$ for some $1 \leq j \leq n_{k}$.

We may assume that the sets $S_{k, 1}, \ldots, S_{k, n_{k}}$ are distinct. From (iii) we have the inclusion $W_{k} \subseteq \bigcup_{j=1}^{n_{k}} \mathcal{V}_{k, j}$. Take an $L^{b} \in \overline{\mathcal{F}}(H)$ such that $\mu\left(X \backslash X_{S^{2}, L^{b}}\right) \leq \Psi(\varepsilon /|\overline{\mathscr{C}}|) /\left(10|S|^{3}\right)$.

Fix $k \in \mathbb{N}$. Put $L_{k}^{*}=L_{k} \cup L^{b} \in \overline{\mathcal{F}}(H)$. Take $0<\delta_{k}^{*} \leq \min \left\{\delta_{k}, \Psi(\varepsilon /|\overline{\mathscr{C}}|) /\left(10|S| \cdot\left|L_{k}^{*}\right|\right)\right\}$. Take also an $L_{k}^{\bullet} \in \overline{\mathcal{F}}(H)$ such that $\mu\left(X \backslash X_{S, L_{k}}\right) \leq \delta_{k}^{*} /(20|S|)$ and an $\bar{L}_{k} \in \overline{\mathcal{F}}(H)$ such that $L_{k}^{\bullet} \subseteq \bar{L}_{k}$ and $\mu\left(X \backslash X_{S, \bar{L}_{k}}\right) \leq \gamma_{k}:=\delta_{k}^{*} /\left(200|S| \cdot\left|L_{k}^{*} L_{k}^{\bullet}\right|\right)$. Put $L_{k}^{\sharp}=\bar{L}_{k} L_{k}^{*} \bar{L}_{k} \in \overline{\mathcal{F}}(H)$. Choose a $T_{k} \in \overline{\mathcal{F}}(G)$ such that $\mu\left(X \backslash X_{L_{k}^{\sharp}, T_{k}}\right) \leq \kappa_{k}:=\delta_{k}^{*} /\left(10^{4}\left|\bar{L}_{k}\right|^{2} \cdot\left|L_{k}^{*} L_{k}^{\bullet}\right|\right)$. Then we have $n_{k}, C_{k}, S_{k, j}$ for $1 \leq j \leq n_{k}, W_{k}$, and $\mathcal{V}_{k, j}$ for $1 \leq j \leq n_{k}$ as above.

Say $S_{k, j}=\bar{F}_{\ell_{k, j}}$ for $1 \leq j \leq n_{k}$. Take an $L_{k}^{\dagger} \in \overline{\mathcal{F}}(H)$ such that $\bigcup_{j=1}^{n_{k}} \Gamma\left(S_{k, j}, \varepsilon / 2^{\ell_{k, j}}\right) \subseteq L_{k}^{\dagger}$ and

$$
\mu\left(X \backslash X_{\bigcup_{j=1}^{n_{k}} S_{k, j}, L_{k}^{\dagger}}\right) \leq \zeta_{k}:=\kappa_{k} /\left(100\left|\bigcup_{j=1}^{n_{k}} S_{k, j}\right|^{C_{k}}\right),
$$

and take $0<\bar{\tau}_{k} \leq \min \left\{\tau_{k}, \kappa_{k} /\left(10 C_{k}\left|\bigcup_{j=1}^{n_{k}} S_{k, j}\right|^{C_{k}} \cdot\left|L_{k}^{\dagger}\right|^{3 C_{k}}\right)\right\}$. Take an $F_{k}^{\natural} \in \overline{\mathcal{F}}(G)$ such that

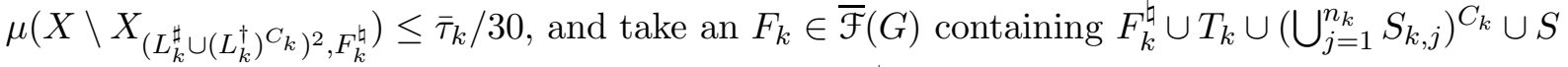

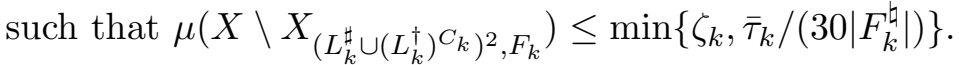

Set $\mathscr{C}_{k}^{\prime}=\left\{W_{k}, X \backslash W_{k}\right\}$ and $\mathscr{C}_{k}^{\prime \prime}=\overline{\mathscr{C}} \vee \mathscr{C}_{k}^{\prime} \vee S_{S^{2}, L^{\mathrm{b}}} \mathscr{P}$. Applying Lemma 4.5 with $\mathscr{C}=\overline{\mathscr{C}}, S=S$, $W=W_{k}$, and $L=L^{\mathrm{b}}$ we find a finite Borel partition $\mathscr{Q}_{k}$ of $W_{k}$ contained in $\operatorname{alg}\left(\left(\overline{\mathscr{C}} \vee \mathscr{C}_{k}^{\prime}\right)_{S^{2}}\right)$ and a map $\Theta_{k}: \mathscr{Q}_{k} \rightarrow \mathcal{F}(S)$ such that $e_{G} \in \Theta_{k}(B)$ for every $B \in \mathscr{Q}_{k}$ and the sets $g B$ for $B \in \mathscr{Q}_{k}$ and $g \in \Theta_{k}(B)$ form a partition $\mathscr{R}_{k}$ of $X$ finer than $\overline{\mathscr{C}}$. Set $\mathscr{C}_{k}^{*}=\mathscr{R}_{k} \vee \mathscr{C}_{k}^{\prime \prime} \vee \mathscr{U}_{k}$.

Denote by $\mathscr{D}_{k}^{\prime}$ the partition of $X$ generated by $W_{k}, \mathcal{V}_{k, 1}, \ldots, \mathcal{V}_{k, n_{k}}$. Put

$$
\mathscr{D}_{k}=\left(\mathscr{C}_{k}^{*}\right)_{L_{k}^{*}} \vee\left(\mathscr{D}_{k}^{\prime}\right)_{T_{k}} \vee\left(\bigvee_{j=1}^{n_{k}} S_{k, j}, L_{k}^{\dagger} \mathscr{P}\right) \vee_{\bigcup_{j=1}^{n_{k}} S_{k, j}, L_{k}^{\dagger}} \mathscr{P} \vee_{L_{k}^{\sharp}, T_{k}} \mathscr{P} \vee_{S, \bar{L}_{k}} \mathscr{P} .
$$

Applying Lemma 4.6 first with $S=S, W=W_{k}, \Theta=\Theta_{k}, \mathscr{Q}=\mathscr{Q}_{k}$, and $\mathscr{D}=\mathscr{D}_{k}$ to get partitions $\mathscr{Q}_{k, 1}$ and $\mathscr{R}_{k, 1}$ and then again with $S=S, W=W_{k}, \Theta=\Theta_{k}, \mathscr{Q}=\mathscr{Q}_{k, 1}$, and $\mathscr{D}=\left(\mathscr{R}_{k, 1}\right)_{L_{k}^{*} \bar{L}_{k}}$, we find finite Borel partitions $\mathscr{Q}_{k, 1}$ and $\mathscr{Q}_{k, 2}$ of $W_{k}$ such that $\mathscr{Q}_{k} \preceq \mathscr{Q}_{k, 1} \preceq \mathscr{Q}_{k, 2}$, and $\mathscr{D}_{k} \preceq \mathscr{R}_{k, 1}$ and $\left(\mathscr{R}_{k, 1}\right)_{L_{k}^{*} \bar{L}_{k}} \preceq \mathscr{R}_{k, 2}$, where for $i=1,2$ we set $\Theta_{k}\left(B_{i}\right)=\Theta_{k}(B)$ for $B \in \mathscr{Q}$ and $B_{i}$ in $\mathscr{Q}_{k, i}$ satisfying $B_{i} \subseteq B$, and $\mathscr{R}_{k, i}$ is the partition of $X$ consisting of the sets $g B_{i}$ for $B_{i} \in \mathscr{Q}_{k, i}$ and $g \in \Theta_{k}\left(B_{i}\right)$. Denote by $\mathscr{Q}_{k, 1}^{\prime}$ the set of all $B \in \mathscr{Q}_{k, 1}$ satisfying $B \subseteq X_{S, L_{k}^{\bullet}}$, and denote by $\mathscr{Q}_{k, 2}^{\prime}$ 
the set of all $B \in \mathscr{Q}_{k, 2}$ satisfying $B \subseteq X_{S, \bar{L}_{k}}$. Denote by $\Lambda_{k}$ the set consisting of the pairs $(B, g)$ for all $B \in \mathscr{Q}_{k, 2}^{\prime}$ and $g \in \Theta_{k}(B) \backslash\left\{e_{G}\right\}$.

Let $1 \leq j \leq n_{k}$. Put $L_{k, j}^{\dagger}=\Gamma\left(S_{k, j}, \varepsilon / 2^{\ell_{k, j}}\right)$. Since $L_{k, j}^{\dagger} \subseteq L_{k}^{\dagger}$, we have $S_{k, j}, L_{k, j}^{\dagger} \overline{\mathscr{P}} \subseteq S_{S_{k, j}, L_{k}^{\dagger}} \overline{\mathscr{P}}$. Denote by $\mathscr{W}_{k, j}$ the finite partition of $X$ consisting of $X_{S_{k, j}, L_{k, j}^{\dagger}}, X \backslash X_{S_{k, j}, L_{k}^{\dagger}}$, and the elements

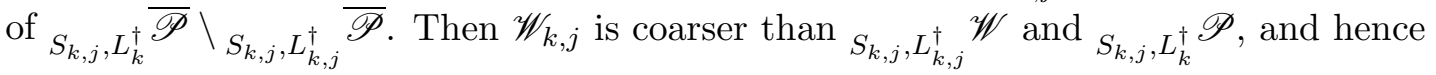

$$
H_{\mu}\left(\mathscr{W}_{k, j}\right) \leq H_{\mu}\left({ }_{S_{k, j}, L_{k, j}^{\dagger}} \mathscr{W}\right) \leq \frac{\varepsilon}{2^{\ell_{k, j}}} .
$$

By [24, Proposition 10.2] we can find an $\eta_{k, j}>0$ such that for any large enough finite set $V$ the number of homomorphisms $\varphi: \operatorname{alg}\left(\mathscr{W}_{k, j}\right) \rightarrow \mathbb{P}_{V}$ satisfying $\sum_{A \in \mathscr{W}_{k, j}}|\mathrm{~m}(\varphi(A))-\mu(A)| \leq \eta_{k, j}$ is

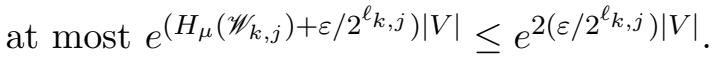

Take

$$
0<\tau_{k}^{\prime} \leq \min \left\{\kappa_{k} /\left(100\left|F_{k}\right|^{3}\right), \bar{\tau}_{k} /\left(60\left|F_{k}\right|^{2}\right)\right\}
$$

and

$$
\begin{gathered}
0<\delta_{k}^{\prime} \leq \min \left\{\kappa_{k} /\left(10 n_{k}\left|F_{k}\right|\right), \delta_{k}^{*} /\left(50\left|L_{k}^{*} L_{k}^{\bullet}\right|(|S|+1)\right), \tau_{k}^{\prime},\right. \\
\left.\Psi(\varepsilon) / 2, \min _{1 \leq j \leq n_{k}} 1 / \Upsilon\left(S_{k, j}\right), \min _{1 \leq j \leq n_{k}} \eta_{k, j}\right\} .
\end{gathered}
$$

Let $\mathscr{A}_{k}$ be a finite Borel partition of $X$ refining

$$
\left(\mathscr{R}_{k, 2}\right)_{\left(L_{k}^{\sharp} \cup\left(L_{k}^{\dagger}\right)^{C_{k}}\right)^{2}} \vee\left(L_{k}^{\sharp} \cup\left(L_{k}^{\dagger}\right)^{\left.C_{k}\right)^{2}, F_{k}} \mathscr{P} \vee\left(\mathscr{D}_{k}\right)_{\left(\bigcup_{j=1}^{n_{k}} S_{k, j}\right)^{C_{k}}}\right.
$$

Take $m_{k} \geq k$ large enough so that

$$
\frac{1}{\left|V_{m_{k}}\right|} \log \left|\operatorname{Hom}_{\mu}\left(\mathscr{A}_{k}, F_{k}, \delta_{k}^{\prime}, \pi_{m_{k}}\right)\right|_{\overline{\mathscr{C}}} \geq h_{\Pi, \mu}(G \curvearrowright X, \overline{\mathscr{C}})-\varepsilon
$$

and so that $\pi_{m_{k}}: G \rightarrow \operatorname{Sym}\left(V_{m_{k}}\right)$ is an $\left(F_{k}, \tau_{k}^{\prime}\right)$-approximation for $G$.

Pick a subset $\Phi$ of $\operatorname{Hom}_{\mu}\left(\mathscr{A}_{k}, F_{k}, \delta_{k}^{\prime}, \pi_{m_{k}}\right)$ such that different elements of $\Phi$ have different restrictions to $\overline{\mathscr{C}}$ and

$$
|\Phi|=\left|\operatorname{Hom}_{\mu}\left(\mathscr{A}_{k}, F_{k}, \delta_{k}^{\prime}, \pi_{m_{k}}\right)\right|_{\mathscr{C}}
$$

Take a maximal subset $\Phi_{1}$ of $\Phi$ which is $\left(\rho_{\overline{\mathscr{C}}}, \Psi(\varepsilon /|\overline{\mathscr{C}}|)\right)$-separated in the sense that $\rho_{\overline{\mathscr{C}}}(\varphi, \psi)>$ $\Psi(\varepsilon /|\overline{\mathscr{C}}|)$ for all distinct $\varphi, \psi \in \Phi_{1}$. For each $\varphi \in \Phi_{1}$, if $\psi \in \Phi$ satisfies $\rho_{\overline{\mathscr{C}}}(\varphi, \psi) \leq \Psi(\varepsilon /|\overline{\mathscr{C}}|)$ then for each $A \in \overline{\mathscr{C}}$ the number of possibilities for $\psi(A)$ is at most $e^{\varepsilon\left|V_{m_{k}}\right| /|\overline{\mathscr{C}}|}$ since $\mathrm{m}(\varphi(A) \Delta \psi(A)) \leq$ $\Psi(\varepsilon /|\overline{\mathscr{C}}|)$. Thus for each $\varphi \in \Phi_{1}$ the number of $\psi \in \Phi$ satisfying $\rho_{\mathscr{C}}(\varphi, \psi) \leq \Psi(\varepsilon /|\overline{\mathscr{C}}|)$ is at most $e^{\varepsilon\left|V_{m_{k}}\right|}$. Therefore

$$
|\Phi| \leq\left|\Phi_{1}\right| e^{\varepsilon\left|V_{m_{k}}\right|}
$$

For every $1 \leq j \leq n_{k}$ and $\varphi \in \Phi_{1}$ we have

$$
\mathrm{m}\left(\varphi\left(\mathcal{V}_{k, j}\right)\right) \leq \mu\left(\mathcal{V}_{k, j}\right)+\delta_{k}^{\prime} \leq \frac{1}{\Upsilon\left(S_{k, j}\right)}+\delta_{k}^{\prime} \leq \frac{2}{\Upsilon\left(S_{k, j}\right)} \leq \Psi\left(\varepsilon /\left(\left.2^{\ell_{k, j}}\right|_{S_{k, j}, L_{k, j}^{\dagger}} \mathscr{P} \mid\right)\right) .
$$

Thus for every $1 \leq j \leq n_{k}$ and $D \in \in_{S_{k, j}, L_{k, j}^{\dagger}} \mathscr{P}$, the number of possibilities for $\varphi\left(\mathcal{V}_{k, j} \cap D\right)$ for $\varphi \in \Phi_{1}$ is at most $e^{\varepsilon\left|V_{m_{k}}\right| /\left(\left.2^{\ell k, j}\right|_{S_{k, j}, L_{k, j}^{\dagger}} \mathscr{P} \mid\right)}$. We can then find a subset $\Phi_{2}$ of $\Phi_{1}$ such that for 
every $1 \leq j \leq n_{k}$ and $D \in S_{S_{k, j}, L_{k, j}^{\dagger}} \mathscr{P}$ the set $\varphi\left(\mathcal{V}_{k, j} \cap D\right)$ is the same for all $\varphi \in \Phi_{2}$ and

$$
\left|\Phi_{1}\right| \leq\left|\Phi_{2}\right| \prod_{j=1}^{n_{k}} e^{\varepsilon\left|V_{m_{k}}\right| / 2^{\ell_{k, j}}} \leq\left|\Phi_{2}\right| e^{\varepsilon\left|V_{m_{k}}\right|} .
$$

In particular, the sets $\varphi\left(\mathcal{V}_{k, j}\right)$ for $1 \leq j \leq n_{k}$ are the same for all $\varphi \in \Phi_{2}$.

Since $\Upsilon \geq 2 / \Psi(\varepsilon)$, for each $\varphi \in \Phi_{2}$ we have

$$
\mathrm{m}\left(\varphi\left(W_{k}\right)\right) \leq \mu\left(W_{k}\right)+\delta_{k}^{\prime} \leq \sum_{j=1}^{n_{k}} \mu\left(\mathcal{V}_{k, j}\right)+\delta_{k}^{\prime} \leq \frac{\Psi(\varepsilon)}{2}+\delta_{k}^{\prime} \leq \Psi(\varepsilon) .
$$

Thus the number of possibilities of $\varphi\left(W_{k}\right)$ for $\varphi \in \Phi_{2}$ is at most $e^{\varepsilon\left|V_{m_{k}}\right|}$. It follows that there is a subset $\Phi_{3}$ of $\Phi_{2}$ such that $\varphi\left(W_{k}\right)$ is the same for all $\varphi \in \Phi_{3}$ and

$$
\left|\Phi_{2}\right| \leq\left|\Phi_{3}\right| e^{\varepsilon\left|V_{m_{k}}\right|} .
$$

For each $1 \leq j \leq n_{k}$, since $\delta_{k}^{\prime} \leq \eta_{k, j}$ the number of possibilities for $\left.\varphi\right|_{\mathscr{W}_{k, j}}$ for $\varphi \in \Phi_{3}$ is at most $e^{2\left(\varepsilon / 2^{\ell, j}\right)\left|V_{m_{k}}\right|}$. Thus there is a subset $\Phi_{4}$ of $\Phi_{3}$ such that for each $1 \leq j \leq n_{k}$ the restriction $\left.\varphi\right|_{\mathscr{W}_{k, j}}$ is the same for all $\varphi \in \Phi_{4}$ and

$$
\left|\Phi_{3}\right| \leq\left|\Phi_{4}\right| \prod_{j=1}^{n_{k}} e^{2\left(\varepsilon / 2^{\ell_{k, j}}\right)\left|V_{m_{k}}\right|} \leq\left|\Phi_{4}\right| e^{2 \varepsilon\left|V_{m_{k}}\right|} .
$$

Note that the set $\varphi\left(W_{k}\right)$ is the same for all $\varphi \in \Phi_{4}$, and for every $1 \leq j \leq n_{k}$ and $D \in \in_{S_{k, j}, L_{k}^{\dagger}} \mathscr{P}$ the set $\varphi\left(\mathcal{V}_{k, j} \cap D\right)$ is the same for all $\varphi \in \Phi_{4}$.

Fix a $\varphi_{0} \in \Phi_{4}$. For each $t \in\left(L_{k}^{\sharp} \cup\left(L_{k}^{\dagger}\right)^{C_{k}}\right)^{2}$ take a map $\sigma_{k, t}^{\prime}: V_{m_{k}} \rightarrow V_{m_{k}}$ such that

$$
\sigma_{k, t}^{\prime} v=\pi_{m_{k}, \lambda(t, A)} v
$$

for all $A \in{ }_{\left(L_{k}^{\sharp} \cup\left(L_{k}^{\dagger}\right)^{C_{k}}\right)^{2}, F_{k}} \overline{\mathscr{P}}$ and $v \in \varphi_{0}(A)$. Applying Lemma 4.2 with $L=L_{k}^{\sharp} \cup\left(L_{k}^{\dagger}\right)^{C_{k}}$, $\tau=\bar{\tau}_{k}, F^{\natural}=F_{k}^{\natural}, F=F_{k}, \tau^{\prime}=\tau_{k}^{\prime}, \pi=\pi_{m_{k}}, \sigma^{\prime}=\sigma_{k}^{\prime}$, and $\varphi$ being the restriction of $\varphi_{0}$ to $\operatorname{alg}\left(\left(\left(_{k}^{\sharp} \cup\left(L_{k}^{\dagger}\right)^{C_{k}}\right)^{2}, F_{k} \mathscr{P}\right)_{F_{k}}\right)$, we find an $\left(L_{k}^{\sharp} \cup\left(L_{k}^{\dagger}\right)^{C_{k}}, \bar{\tau}_{k}\right)$-approximation $\sigma_{k}: H \rightarrow \operatorname{Sym}\left(V_{m_{k}}\right)$ for $H$ such that $\rho_{\text {Hamm }}\left(\sigma_{k, t}, \sigma_{k, t}^{\prime}\right) \leq \bar{\tau}_{k} / 5$ for all $t \in\left(L_{k}^{\sharp} \cup\left(L_{k}^{\dagger}\right)^{C_{k}}\right)^{2}$.

Let $\varphi \in \Phi_{4}$. Define $\tilde{\varphi}^{\prime}: \mathscr{R}_{k, 2} \rightarrow \mathbb{P}_{V_{m_{k}}}$ by $\tilde{\varphi}^{\prime}(B)=\varphi(B)$ for all $B \in \mathscr{Q}_{k, 2}, \tilde{\varphi}^{\prime}(g B)=\emptyset$ for all $B \in \mathscr{Q}_{k, 2} \backslash \mathscr{Q}_{k, 2}^{\prime}$ and $g \in \Theta_{k}(B) \backslash\left\{e_{G}\right\}$, and

$$
\tilde{\varphi}^{\prime}(g B)=\sigma_{k, \kappa(g, B)} \varphi(B)
$$

for all $(B, g) \in \Lambda_{k}$. Extend $\tilde{\varphi}^{\prime}$ to a map $\operatorname{alg}\left(\mathscr{R}_{k, 2}\right) \rightarrow \mathbb{P}_{V_{m_{k}}}$ by setting $\tilde{\varphi}^{\prime}(D)=\bigcup_{A \in \mathscr{R}_{k, 2}, A \subseteq D} \tilde{\varphi}^{\prime}(A)$ for $D \in \operatorname{alg}\left(\mathscr{R}_{k, 2}\right)$. Applying Lemma 4.8 with $\mathscr{C}=\mathscr{C}_{k}^{*}, L=L_{k}^{*}, \delta=\delta_{k}^{*}, S=S, L^{\bullet}=L_{k}^{\bullet}, \bar{L}=\bar{L}_{k}$, $T=T_{k}, C=C_{k}, n=n_{k}, S_{j}=S_{k, j}, W=W_{k}, \mathcal{V}_{j}=\mathcal{V}_{k, j}, L^{\dagger}=L_{k}^{\dagger}, \bar{\tau}=\bar{\tau}_{k}, F=F_{k}, \mathscr{Q}_{1}=\mathscr{Q}_{k, 1}$, $\mathscr{Q}_{2}=\mathscr{Q}_{k, 2}, \Theta=\Theta_{k}, \tau^{\prime}=\tau_{k}^{\prime}, \delta^{\prime}=\delta_{k}^{\prime}, \mathscr{A}=\mathscr{A}_{k}, \pi=\pi_{m_{k}}, \varphi=\varphi, \varphi_{0}=\varphi_{0}$, and $\sigma=\sigma_{k}$, by our choice of $\Phi_{4}$ we have

$$
\sum_{(B, g) \in \Lambda_{k}} \mathrm{~m}\left(\tilde{\varphi}^{\prime}(g B) \cap \varphi\left(W_{k}\right)\right) \leq \frac{\delta_{k}^{*}}{40}
$$




$$
\sum_{\substack{(B, g),\left(B^{\prime}, g^{\prime}\right) \in \Lambda_{k},(B, g) \neq\left(B^{\prime}, g^{\prime}\right)}} \mathrm{m}\left(\tilde{\varphi}^{\prime}(g B) \cap \tilde{\varphi}^{\prime}\left(g^{\prime} B^{\prime}\right)\right) \leq \frac{\delta_{k}^{*}}{40},
$$

and

$$
\sum_{B \in \mathscr{Q}_{k, 1}^{\prime}} \sum_{t \in L_{k}^{*} L_{k}^{\bullet}} \mathrm{m}\left(\tilde{\varphi}^{\prime}(t B) \Delta \sigma_{k, t} \varphi(B)\right) \leq \frac{\delta_{k}^{*}}{20} .
$$

Applying Lemma 4.7 with $\mathscr{C}=\mathscr{C}_{k}^{*}, L=L_{k}^{*}, \delta=\delta_{k}^{*}, S=S, W=W_{k}, L^{\bullet}=L_{k}^{\bullet}, \bar{L}=\bar{L}_{k}$, $\mathscr{Q}_{1}=\mathscr{Q}_{k, 1}, \mathscr{Q}_{2}=\mathscr{Q}_{k, 2}, \Theta=\Theta_{k}, \mathscr{A}=\mathscr{A}_{k}, \varphi=\varphi, \delta^{\prime}=\delta_{k}^{\prime}, \bar{\tau}=\bar{\tau}_{k}$, and $\sigma=\sigma_{k}$, we find a homomorphism $\tilde{\varphi}: \operatorname{alg}\left(\mathscr{R}_{k, 2}\right) \rightarrow \mathbb{P}_{V_{m_{k}}}$ such that $\tilde{\varphi}(B)=\varphi(B)$ for every $B \in \mathscr{Q}_{k, 2}$ and $\sum_{A \in \mathscr{R}_{k, 2}} \mathrm{~m}\left(\tilde{\varphi}(A) \Delta \tilde{\varphi}^{\prime}(A)\right) \leq \delta_{k}^{*} / 5$. Furthermore, the restriction of $\tilde{\varphi}$ to $\operatorname{alg}\left(\left(\mathscr{C}_{k}^{*}\right)_{L_{k}^{*}}\right) \operatorname{lies}$ in $\operatorname{Hom}_{\mu}\left(\mathscr{C}_{k}^{*}, L_{k}^{*}, \delta_{k}^{*}, \sigma_{k}\right)$.

For any distinct $\varphi, \psi$ in $\Phi_{4}$, applying Lemma 4.5 with $\mathscr{C}=\overline{\mathscr{C}}, S=S, W=W_{k}, L=L^{b}$, $\mathscr{Q}=\mathscr{Q}_{k}, \pi=\pi_{m_{k}}, \sigma=\sigma_{k}, \delta=\delta_{k}^{*}$, and $\delta^{\prime}=\delta_{k}^{\prime}$ we have

$$
\begin{aligned}
\Psi(\varepsilon /|\overline{\mathscr{C}}|) \leq & \rho_{\overline{\mathscr{C}}}(\varphi, \psi) \\
\leq & 2\left(\delta_{k}^{*}+\delta_{k}^{\prime}\right)|S|+2 \delta_{k}^{*}|S| \cdot\left|L^{b}\right|+2|S|^{3} \mu\left(X \backslash X_{S^{2}, L^{b}}\right) \\
& \quad+|S| \cdot\left|L^{b}\right| \rho_{\overline{\mathscr{C}} \vee_{S^{2}, L^{b}} \mathscr{P}}(\tilde{\varphi}, \tilde{\psi}) \\
\leq & \frac{4}{5} \Psi(\varepsilon /|\overline{\mathscr{C}}|)+|S| \cdot\left|L^{b}\right| \rho_{\overline{\mathscr{C}}_{S^{2}, L^{b}} \mathscr{P}}(\tilde{\varphi}, \tilde{\psi}),
\end{aligned}
$$

and hence

$$
\rho_{\overline{\mathscr{C}} \vee_{S^{2}, L^{b}} \mathscr{P}}(\tilde{\varphi}, \tilde{\psi}) \geq \varepsilon^{\prime}:=\frac{\Psi(\varepsilon /|\overline{\mathscr{C}}|)}{5|S| \cdot\left|L^{b}\right|}
$$

Thus

$$
\begin{aligned}
& \frac{1}{\left|V_{m_{k}}\right|} \log \left|\operatorname{Hom}_{\mu}\left(\overline{\mathscr{C}} \vee_{S^{2}, L^{b}} \mathscr{P} \vee \mathscr{U}_{k}, L_{k}, \delta_{k}, \sigma_{k}\right)\right|_{\mathscr{C} \vee_{S^{2}, L^{b}} \mathscr{P}} \\
& \geq \frac{1}{\left|V_{m_{k}}\right|} \log \left|\operatorname{Hom}_{\mu}\left(\mathscr{C}_{k}^{*}, L_{k}^{*}, \delta_{k}^{*}, \sigma_{k}\right)\right|_{\mathscr{C} \vee_{S^{2}, L^{b}} \mathscr{P}} \\
& \geq \frac{1}{\left|V_{m_{k}}\right|} \log \left|\Phi_{4}\right| \\
& \geq \frac{1}{\left|V_{m_{k}}\right|} \log |\Phi|-5 \varepsilon \\
& \geq h_{\Pi, \mu}(G \curvearrowright X, \overline{\mathscr{C}})-6 \varepsilon .
\end{aligned}
$$

Since $L_{k} \subseteq L_{k}^{\sharp}$ and $\bar{\tau}_{k} \leq \tau_{k}$ for every $k \in \mathbb{N}$, the sequence $\Sigma=\left\{\sigma_{k}\right\}_{k \in \mathbb{N}}$ is a sofic approximation sequence for $H$. Set $\mathscr{C}^{b}=\overline{\mathscr{C}} \vee_{S^{2}, L^{b}} \mathscr{P}$. For any finite partition $\mathscr{U}$ of $X$ contained in the algebra generated by $\bigcup_{k \in \mathbb{N}} \mathscr{U}_{k}$, any $L \in \mathcal{F}(H)$ containing $e_{H}$, and any $\delta>0$, we have $\mathscr{U} \preceq \mathscr{U}_{k}, L \subseteq L_{k}$, and $\delta>\delta_{k}$ for all large enough $k$, and hence

$$
\begin{aligned}
h_{\Sigma, \mu}\left(\mathscr{C}^{b}, \mathscr{C}^{b} \vee \mathscr{U}, L, \delta\right) \\
\quad=\varlimsup_{k \rightarrow \infty} \frac{1}{\left|V_{m_{k}}\right|} \log \left|\operatorname{Hom}_{\mu}\left(\mathscr{C}^{b} \vee \mathscr{U}, L, \delta, \sigma_{k}\right)\right|_{\mathscr{C}^{b}}
\end{aligned}
$$




$$
\begin{aligned}
& \geq \varlimsup_{k \rightarrow \infty} \frac{1}{\left|V_{m_{k}}\right|} \log \left|\operatorname{Hom}_{\mu}\left(\overline{\mathscr{C}} \vee_{S^{2}, L^{\mathrm{b}}} \mathscr{P} \vee \mathscr{U}_{k}, L_{k}, \delta_{k}, \sigma_{k}\right)\right|_{\overline{\mathscr{C}}_{S^{2}, L^{\mathrm{b}}} \mathscr{P}} \\
& \geq h_{\Pi, \mu}(G \curvearrowright X, \overline{\mathscr{C}})-6 \varepsilon .
\end{aligned}
$$

Since the algebra generated by $\bigcup_{k \in \mathbb{N}} \mathscr{U}_{k}$ is dense in the Borel $\sigma$-algebra of $X$ with respect to the pseudometric $d(A, B)=\mu(A \Delta B)$, by [24, Lemma 10.13] we conclude that

$$
h_{\Sigma, \mu}\left(H \curvearrowright X, \mathscr{C}^{b}\right) \geq h_{\Pi, \mu}(G \curvearrowright X, \overline{\mathscr{C}})-6 \varepsilon
$$

as desired.

Remark 4.9. Theorem 4.1, and hence also Theorem A, actually uses only that $\kappa$ is Shannon, not that $\lambda$ is Shannon.

\section{REFERENCES}

[1] M. Abért and B. Weiss. Bernoulli actions are weakly contained in any free action. Ergodic Theory Dynam. Systems 33 (2013), 323-333.

[2] T. Austin. Behaviour of entropy under bounded and integrable orbit equivalence. Geom. Funct. Anal. 26 (2016), 1483-1525.

[3] B. Bekka, P. de la Harpe, and A. Valette. Kazhdan's Property (T). New Mathematical Monographs, 11. Cambridge University Press, Cambridge, 2008.

[4] R. M. Belinskaya. Partitions of Lebesgue space in trajectories defined by ergodic automorphisms. Functional Anal. Appl. 2 (1968), 190-199.

[5] S. I. Bezuglyi and V. Ya. Golodets. Hyperfinite and $\mathrm{II}_{1}$ actions for nonamenable groups. J. Funct. Anal. 40 (1981), 30-44.

[6] L. Bowen. Measure conjugacy invariants for actions of countable sofic groups. J. Amer. Math. Soc. 23 (2010), 217-245.

[7] L. Bowen. Orbit equivalence, coinduced actions and free products. Groups Geom. Dyn. 5 (2011), 1-15.

[8] L. Bowen. Stable orbit equivalence of Bernoulli shifts over free groups. Groups Geom. Dyn. 5 (2011), 17-38.

[9] L. Bowen. Every countably infinite group is almost Ornstein. In: Dynamical Systems and Group Actions, 67-78, Contemp. Math., 567, Amer. Math. Soc., Providence, RI, 2012.

[10] L. Bowen. Sofic entropy and amenable groups. Ergodic Theory Dynam. Systems 32 (2012), 427-466.

[11] R. de Santiago, B. Hayes, D. J. Hoff, and T. Sinclair. Maximal rigid subalgebras of deformations and $L^{2}$-cohomology. arXiv:1909.03570.

[12] I. Epstein. Some results on orbit inequivalent actions of non-amenable groups. Ph.D. thesis, University of California, Los Angeles. 2008. 55 pp.

[13] A. Fieldsteel and N. A. Friedman, Restricted orbit changes of ergodic $\mathbf{Z}^{d}$-actions to achieve mixing and completely positive entropy. Ergodic Theory Dynam. Systems 6 (1986), 505-528.

[14] A. Furman. Orbit equivalence rigidity. Ann. of Math. (2) 150 (1999), 1083-1108.

[15] D. Gaboriau. Coût des relations d'équivalence et des groupes. Invent. Math. 139 (2000), 41-98.

[16] D. Gaboriau and S. Popa. An uncountable family of nonorbit equivalent actions of $\mathbb{F}_{n}$. J. Amer. Math. Soc. 18 (2005), 547-559.

[17] B. Hayes. Fuglede-Kadison determinant and sofic entropy. Geom. Funct. Anal. 26 (2016), 520-606.

[18] G. Hjorth. A converse to Dye's theorem. Trans. Amer. Math. Soc. 357 (2005), 3083-3103.

[19] A. Ioana. Orbit inequivalent actions for groups containing a copy of $\mathbb{F}_{2}$. Invent. Math. 185 (2011), 55-73.

[20] A. S. Kechris. Global Aspects of Ergodic Group Actions. Mathematical Surveys and Monographs, 160. American Mathematical Society, Providence, RI, 2010.

[21] A. S. Kechris and B. D. Miller. Topics in Orbit Equivalence. Lecture Notes in Mathematics, 1852. SpringerVerlag, Berlin, 2004.

[22] D. Kerr and H. Li. Bernoulli actions and infinite entropy. Groups Geom. Dyn. 5 (2011), 663-672.

[23] D. Kerr and H. Li. Soficity, amenability, and dynamical entropy. Amer. J. Math. 135 (2013), 721-761.

[24] D. Kerr and H. Li. Ergodic Theory: Independence and Dichotomies. Springer, Cham, 2016. 
[25] Y. Kida. Orbit equivalence rigidity for ergodic actions of the mapping class group. Geom. Dedicata 131 (2008), 99-109.

[26] W. Lück. $L^{2}$-Invariants: Theory and Applications to Geometry and K-Theory. Ergebnisse der Mathematik und ihrer Grenzgebiete, 3. Folge, vol. 44. Springer-Verlag, Berlin, 2002.

[27] A. Mann. How Groups Grow. London Mathematical Society Lecture Note Series, 395. Cambridge University Press, Cambridge, 2012.

[28] N. Monod and Y. Shalom. Orbit equivalence rigidity and bounded cohomology. Ann. of Math. (2) 164 (2006), 825-878.

[29] D. S. Ornstein and B. Weiss. Entropy and isomorphism theorems for actions of amenable groups. J. Analyse Math. 48 (1987), 1-141.

[30] J. Peterson and T. Sinclair. On cocycle superrigidity for Gaussian actions. Ergodic Theory Dynam. Systems 32 (2012), 249-272.

[31] S. Popa. Cocycle and orbit equivalence superrigidity for malleable actions of w-rigid groups. Invent. Math. 170 (2007), 243-295.

[32] S. Popa. On the superrigidity of malleable actions with spectral gap. J. Amer. Math. Soc. 21 (2008), 981-1000.

[33] B. Seward. Bernoulli shifts with bases of equal entropy are isomorphic. arXiv:1805.08279

[34] A. M. Stepin. Bernoulli shifts on groups. Dokl. Akad. Nauk SSSR 223 (1975), 300-302.

[35] R. Tucker-Drob. Invariant means and the structure of inner amenable groups. To appear in Duke Math. J.

[36] A. M. Vershik. Approximation in Measure Theory. Ph.D. thesis, Leningrad University, 1973. In Russian.

[37] A. M. Vershik. Theory of decreasing sequences of measurable partitions. St. Petersburg Math. J. 6 (1995), $705-761$

[38] R. J. Zimmer. Strong rigidity for ergodic actions of semisimple Lie groups. Ann. of Math. (2) 112 (1980), $511-529$

David Kerr, Department of Mathematics, Texas A\&M University, College Station, TX 778433368, USA

E-mail address: kerr@math.tamu.edu

Hanfeng Li, Center of Mathematics, Chongqing University, Chongqing 40133, China and DePARTMENT of Mathematics, SUNY at Buffalo, Buffalo, NY 14260-2900, USA

E-mail address: hfli@math.buffalo.edu 